Supplementary Information

\title{
Polymerization-Induced Self-assembly Driven by the Synergistic Effects of Aromatic and Solvophobic Interactions
}

Xiao-Fei $\mathrm{Xu}^{\ddagger}$, Ren-Man Zhu ${ }^{\ddagger}$, Cai-Yuan Pan, Ye-Zi You, Wen-Jian Zhang* and Chun-Yan Hong*

CAS Key Laboratory of Soft Matter Chemistry, Department of Polymer Science and Engineering, University of Science and Technology of China, Hefei, 230026, Anhui, P.R. China.

Email: zwj85@ustc.edu.cn

Email: hongcy@ustc.edu.cn 


\section{Experimental Section.}

\section{Materials.}

Methacryloyl chloride (95\%, Aladdin), hydroxyethyl methacrylate (96\%, Aladdin), anthracen-9-carboxylic acid (98\%, Aladdin) and 2-bromoethanol (95 $\%$, Aladdin) were used as received without further purification. 2-(Diisopropylamino)ethyl methacrylate (DIPEMA, $97 \%$, Sigma-Aldrich) was purified by passing through a column of alkaline $\mathrm{Al}_{2} \mathrm{O}_{3}$ to remove the inhibitor prior to use. Polyethylene glycol monomethyl ether 2000 (mPEO) was purchased from $\mathrm{TCl}$ and used as received. N, N'-azobis(isobutyronitrile) (AIBN, Sinopharm Chemical Reagent Co. Ltd) was purified by recrystallization from ethanol. 4-Cyano-4-(phenylcarbonothioylthio)-pentanoic acid (CPADB) was purchased from Sigma-Aldrich. All other chemicals were of analytical grade and used as received.

\section{Synthesis of 7-(2-methacryloyloxyethoxy)-4-methylcoumarin (CMA).}
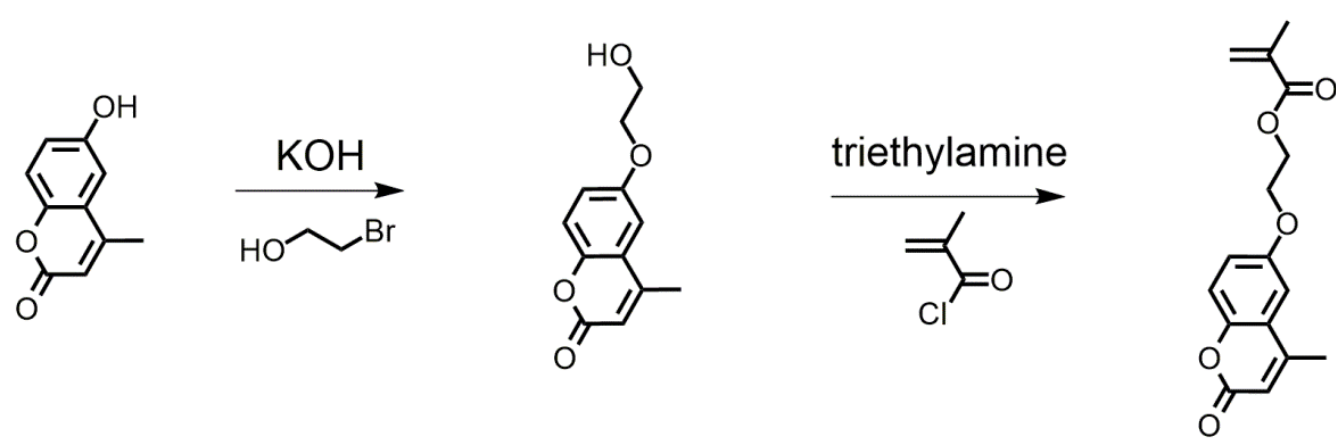
At first, 7-(2-hydroxyethoxy)-4-methylcoumarin was synthesized as the following procedures. The 7-hydroxy-4-methylcoumarin $(5.0 \mathrm{~g}, 28.4 \mathrm{mmol})$ and aqueous solution of potassium hydroxide $(7.0 \mathrm{~mL}, 25 \%$, w/v) were added into $100 \mathrm{~mL}$ of ethanol. After stirring at room temperature for $0.5 \mathrm{~h}, 5.0 \mathrm{~g}$ of 2-bromoethanol was added into the reaction system, then the mixture was heated under reflux for $24 \mathrm{~h}$. After removing the solvent via rotary evaporation, $300 \mathrm{~mL}$ of trichloromethane was added to dissolve the crude product. The solution was washed with water for three times and then dried over anhydrous magnesium sulfate for $12 \mathrm{~h}$. After removing the solid by filtration, the solvent was removed by rotary evaporation to afford some white powder $(77.5 \%$ yield). ${ }^{1} \mathrm{H} \quad \mathrm{NMR}$ spectrum in Figure S3 shows that the product 7-(2-hydroxyethoxy)-4-methylcoumarin was pure enough to be used in the next step without further purification.

The 7-(2-hydroxyethoxy)-4-methylcoumarin $(4.4 \mathrm{~g}, 20.0 \mathrm{mmol})$ and triethylamine $(4.0 \mathrm{~g}, 40.0 \mathrm{mmol})$ dissolved in $\mathrm{CH}_{2} \mathrm{Cl}_{2}(150 \mathrm{~mL})$ was cooled in an ice-water bath for $15 \mathrm{~min}$, and then methacryloyl chloride $(4.2 \mathrm{~g}, 40.0 \mathrm{mmol})$ in $\mathrm{CH}_{2} \mathrm{Cl}_{2}(30 \mathrm{~mL})$ was slowly added into above mixture in about $1 \mathrm{~h}$. The reaction was allowed to proceed for further $24 \mathrm{~h}$ at room temperature. The solution was diluted with $200 \mathrm{~mL}$ of $\mathrm{CH}_{2} \mathrm{Cl}_{2}$ and then washed with distilled water for three times $(3 \times 100 \mathrm{~mL})$. The organic phase was dried over anhydrous $\mathrm{Na}_{2} \mathrm{SO}_{4}$ overnight. After removing the solvent by rotary evaporation, the crude product was purified by column chromatography using 
$\mathrm{CHCl}_{3}$ as the running solvent. White powder was obtained in $85 \%$ yield. ${ }^{1} \mathrm{H}$ NMR and ${ }^{13} \mathrm{C}$ NMR spectra shown in Figure S4 and S5 indicated successful synthesis of the 7-(2-methacryloyloxyethoxy)-4-methylcoumarin (CMA).

\section{Synthesis of 2-(methacryloyloxy)ethyl anthracene-9-carboxylate} (ACMAE).

1)<smiles></smiles><smiles>O=S(Cl)Cl</smiles><smiles>[CH2-]</smiles><smiles></smiles>

2)<smiles></smiles><smiles>C=C(C)C(=O)OCO</smiles><smiles>C=C(C)C(=O)OCOC(=O)c1c2ccccc2cc2ccccc12</smiles>

Anthracen-9-carboxylic acid $(7.03 \mathrm{~g}, 31.7 \mathrm{mmol})$ and thionyl chloride $(70 \mathrm{~mL})$ were mixed together. After being completely dissolved, the above mixture was heated to $70^{\circ} \mathrm{C}$ at oil bath while stirring, and the reaction was conducted under reflux condensation for $12 \mathrm{~h}$. After removing the residual thionyl chloride by rotary evaporation, the crude product was dissolved in $30 \mathrm{~mL}$ anhydrous tetrahydrofuran and then added dropwise into a mixture of triethylamine (3.94 $\mathrm{g}, 39.0 \mathrm{mmol})$, hydroxyethyl methacrylate $(2.78 \mathrm{~g}, 21.5 \mathrm{mmol})$ and anhydrous tetrahydrofuran $(100 \mathrm{~mL})$ in about $1 \mathrm{~h}$ at $0{ }^{\circ} \mathrm{C}$. The reaction was allowed to 
proceed for $20 \mathrm{~h}$ at room temperature, and then tetrahydrofuran was removed by rotary evaporation. The obtained product was dissolved in $200 \mathrm{~mL}$ dichloromethane and washed with $2 \mathrm{M} \mathrm{HCl}(2 \times 100 \mathrm{~mL}), 2 \mathrm{M} \mathrm{Na}_{2} \mathrm{CO}_{3}(4 \times 100$ $\mathrm{mL})$ and distilled water $(3 \times 100 \mathrm{~mL})$ successively. The organic phase was dried over anhydrous $\mathrm{Na}_{2} \mathrm{SO}_{4}$ overnight. After removing the solvent by rotary evaporation, the crude product was purified by column chromatography using mixture of petroleum ether and dichloromethane $(1: 1, \mathrm{v} / \mathrm{v})$ as the running solvent. ${ }^{1} \mathrm{H}$ NMR and ${ }^{13} \mathrm{C}$ NMR spectrum shown in Figure S16 and S17 indicated successful synthesis of 2-(methacryloyloxy)ethyl anthracene-9-carboxylate (ACMAE).

\section{Synthesis of macro-RAFT agent PEO 45 -CPADB.}

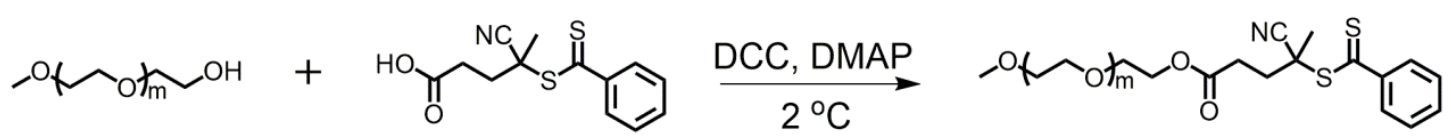

$\mathrm{mPEO}_{45} \quad(5 \mathrm{~g}, \quad 2.5 \mathrm{mmol}), \quad$ CPADB $(2.79 \mathrm{~g}, \quad 10 \mathrm{mmol})$ and 4-dimethylaminopyridine $(0.122 \mathrm{~g}, 1 \mathrm{mmol})$ were dissolved in $75 \mathrm{~mL}$ of anhydrous dichloromethane and then stirred at $2{ }^{\circ} \mathrm{C}$ for $0.5 \mathrm{~h}$. Dicyclohexylcarbodiimide $(2.06 \mathrm{~g}, 10 \mathrm{mmol})$ in $25 \mathrm{~mL}$ anhydrous dichloromethane was added dropwise into the above mixture. The reaction was carried out at $2{ }^{\circ} \mathrm{C}$ for 3 days and then the mixture was filtered to remove 
$\mathrm{N}, \mathrm{N}$-dicyclohexylurea. Most of the dichloromethane was removed by rotary evaporator before precipitation in cold diethyl ether. The polymer was collected by filtration and the above precipitation procedure was repeated for three times. The product of pink powder $(5.0 \mathrm{~g})$ in $88 \%$ yield was obtained after drying under vacuum overnight.

\section{RAFT dispersion polymerization of CMA using PEO ${ }_{45}$-CPADB as the macro-RAFT agent.}

The PEO45-CPADB macro-RAFT agent mediated RAFT dispersion polymerization of CMA was conducted in the ethanol/water mixture $(70 / 30$, $\mathrm{w} / \mathrm{w})$ at $70^{\circ} \mathrm{C}$ under $\left[\mathrm{PEO}{ }_{45}-\mathrm{CPADB}\right]_{0}:[\text { initiator }]_{0}=5: 1$ with $5 \mathrm{wt} \%$ solid content. A typical polymerization under [monomer $]_{0}:\left[P E{ }_{45}-\mathrm{CPADB}\right]_{0}:[\text { initiator }]_{0}=80$ : 1: $1 / 5$ was introduced here. PEO $45-C P A D B\left(92 \mathrm{mg}, 4 \times 10^{-2} \mathrm{mmol}\right.$ ), CMA (922 $\mathrm{mg}, 3.2 \mathrm{mmol})$, AIBN $\left(0.13 \mathrm{mg}, 0.8 \times 10^{-3} \mathrm{mmol}\right)$ and solvent $(19.3 \mathrm{~g}$, the mass ratio of ethanol/water $=7 / 3$ ) were weighed into a glass bottle with a magnetic stir bar. The mixture was degassed by $\mathrm{N}_{2}$ for $30 \mathrm{~min}$. The polymerization was carried out at $70^{\circ} \mathrm{C}$, and $0.5 \mathrm{~mL}$ of the sample was taken out by a syringe at predetermined time interval for NMR, GPC and TEM characterization. 
RAFT dispersion copolymerization of CMA and DIPEMA to synthesize the

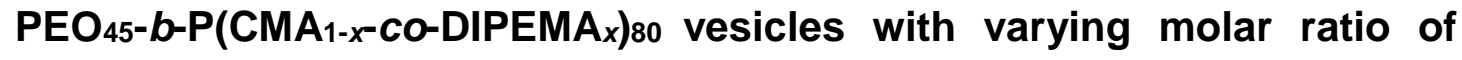
DIPEMA but a fixed DPP(CMA-co-DIPEMA) at 80.

The dispersion copolymerization of CMA and DIPEMA with varying molar ratio of DIPEMA but a fixed DPP(CMA-co-DIPEMA) at 80 was conducted in the ethanol/water mixture $(70 / 30, \mathrm{w} / \mathrm{w})$ at $70^{\circ} \mathrm{C}$ at $5 \mathrm{wt} \%$ solid content. The details in feeds for the fabrication of the PEO45- $b-\mathrm{P}\left(\mathrm{CMA}_{1-x} \text { Co-DIPEMA }\right)_{80}$ vesicles are summarized in Table S1. Herein, a typical copolymerization of CMA and DIPEMA with $x$ at 0.25 is introduced. PEO 45 -CPADB $\left(11 \mathrm{mg}, 5 \times 10^{-3} \mathrm{mmol}\right)$, CMA (86 mg, $0.30 \mathrm{mmol})$, DIPEMA (21 mg, $0.1 \mathrm{mmol})$, AIBN (0.16 mg, 10-3 $\mathrm{mmol})$ and solvent $(2.24 \mathrm{~g}$, the mass ratio of ethanol/water $=7 / 3)$ were weighed into a glass tube with a magnetic stir bar. After three freeze-pump-thaw cycles, the tube was sealed, and then polymerization was carried out at $70^{\circ} \mathrm{C}$ for $12 \mathrm{~h}$ under stirring.

RAFT dispersion copolymerization of CMA and BzMA to synthesize the

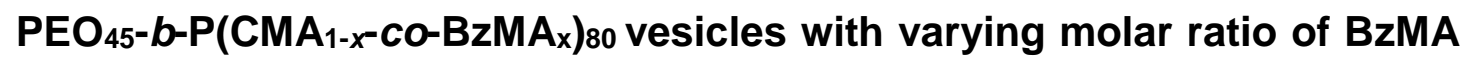
but a fixed DPP(CMA-co-BzMA) at 80 .

The dispersion copolymerization of CMA and BzMA with varying molar ratio of BzMA but a fixed DP $P_{P(C M A-c o-B z M A)}$ at 80 was conducted in the ethanol/water mixture $(70 / 30, w / w)$ at $70^{\circ} \mathrm{C}$ at $5 \mathrm{wt} \%$ solid content. The details in feeds for 
the fabrication of the $\mathrm{PEO}_{45}-b-\mathrm{P}\left(\mathrm{CMA}_{1-x}-\mathrm{co}-\mathrm{BzMA}\right) 80$ vesicles are summarized in Table S3. Herein, a typical copolymerization of CMA and BzMA with $x$ at 0.25 is introduced. PEO $45-$ CPADB (11 mg, $5 \times 10^{-3} \mathrm{mmol}$ ), CMA (86 mg, 0.30 mmol), BzMA (18 mg, $0.1 \mathrm{mmol}), \operatorname{AIBN}\left(0.16 \mathrm{mg}, 10^{-3} \mathrm{mmol}\right)$ and solvent $(2.19$ $\mathrm{g}$, the mass ratio of ethanol/water $=7 / 3$ ) were weighed into a glass tube with a magnetic stir bar. After three freeze-pump-thaw cycles, the tube was sealed, and then polymerization was carried out at $70{ }^{\circ} \mathrm{C}$ for $12 \mathrm{~h}$ under stirring.

RAFT dispersion copolymerization of CMA and ACMAE to synthesize the

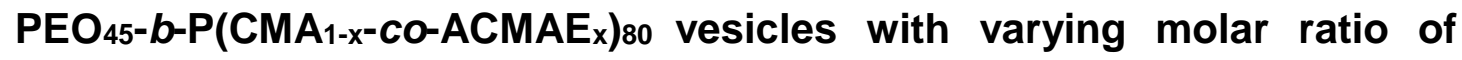
ACMAE but a fixed DPP(CMA-co-ACMAE) at 80 .

The dispersion copolymerization of CMA and ACMAE with varying molar ratio of ACMAE but a fixed DPP(CMA-co-ACMAE) at 80 was conducted in the ethanol/water mixture $(70 / 30, w / w)$ at $70{ }^{\circ} \mathrm{C}$ at $5 \mathrm{wt} \%$ solid content. The details in feeds for the fabrication of the $\mathrm{PEO}_{45}-b-\mathrm{P}\left(\mathrm{CMA}_{1-\mathrm{x}}-\mathrm{Co}-\mathrm{ACMAE}_{\mathrm{x}}\right)_{80}$ vesicles are summarized in Table S5. Herein, a typical copolymerization of CMA and ACMAE with $x$ at 0.25 is introduced. PEO 45 -CPADB (11 $\mathrm{mg}, 5 \times 10^{-3} \mathrm{mmol}$ ), CMA (86 mg, $0.30 \mathrm{mmol}), \operatorname{ACMAE}(33 \mathrm{mg}, 0.1 \mathrm{mmol}), \operatorname{AIBN}\left(0.16 \mathrm{mg}, 10^{-3}\right.$ $\mathrm{mmol}$ ) and solvent $(2.47 \mathrm{~g}$, the mass ratio of ethanol/water $=7 / 3$ ) were weighed into a glass tube with a magnetic stir bar. After three 
freeze-pump-thaw cycles, the tube was sealed, and then polymerization was carried out at $70^{\circ} \mathrm{C}$ for $12 \mathrm{~h}$ under stirring.

RAFT dispersion polymerization of CMA to fabricate the $\mathrm{PEO}_{45}-b-\mathrm{PCMA}_{\mathrm{y}}$ vesicles with varying DPpсmA $(100,120,150,200)$.

RAFT dispersion polymerization of CMA with varying DPPCMA (100, 120, 150, 200) were carried out at $5 \%$ or $10 \%$ solid content, or fixed chain concentration of $3.01 \times 10^{-3} \mathrm{mmol} / \mathrm{g}$. The details in feeds for the fabrication of the

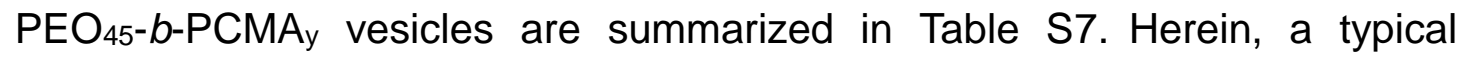
procedure of fabrication of $\mathrm{PEO}_{45}-b-\mathrm{PCMA}_{200}$ vesicles at $10 \%$ solid content is introduced. PEO $45-$ CPADB (11 mg, $\left.5 \times 10^{-3} \mathrm{mmol}\right)$, CMA (288 mg, $1 \mathrm{mmol}$ ), AIBN $\left(0.16 \mathrm{mg}, 10^{-3} \mathrm{mmol}\right)$ and solvent $(2.69 \mathrm{~g}$, the mass ratio of ethanol/water $=7 / 3$ ) were weighed into a glass tube with a magnetic stir bar. After three freeze-pump-thaw cycles, the tube was sealed, and then polymerization was carried out at $70^{\circ} \mathrm{C}$ for $18 \mathrm{~h}$ under stirring.

RAFT dispersion copolymerization of CMA and comonomer M (DIPEMA, BzMA or ACMAE) to synthesize the $\mathrm{PEO}_{45}-\mathrm{b}-\mathrm{P}\left(\mathrm{CMA} \mathrm{A}_{0.75}-\mathrm{co}-\mathrm{M}_{0.25}\right)$ y vesicles with varying DP of the solvophobic block $(80,100,120,150,200)$ but a fixed molar content of CMA in the solvophobic block at $75 \%$. 
The dispersion copolymerization of CMA and comonomer $M$ (DIPEMA, BzMA or ACMAE) to synthesize the $\mathrm{PEO}_{45}-b-\mathrm{P}\left(\mathrm{CMA}_{\left.0.75-C o-M_{0.25}\right) \text { v vesicles }}\right.$ with varying DP of the solvophobic block $(80,100,120,150,200)$ was carried out in the ethanol/water mixture $(70 / 30, \mathrm{w} / \mathrm{w})$ at $70{ }^{\circ} \mathrm{C}$ at a fixed chain concentration of $3.01 \times 10^{-3} \mathrm{mmol} / \mathrm{g}$. The details in feeds for the fabrication of

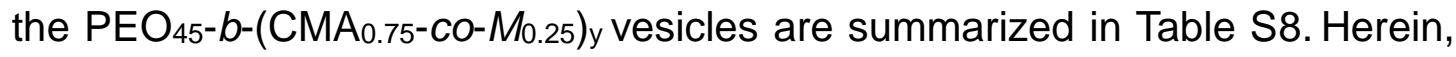
a typical copolymerization of CMA and ACMAE to fabricate the $\mathrm{PEO}_{45}-b-\mathrm{P}\left(\mathrm{CMA}_{0.75}-\mathrm{Co}-\mathrm{ACMAE}_{0.25}\right)_{200}$ vesicles is introduced. $\mathrm{PEO}_{45}-\mathrm{CPADB}$ (11 mg, $\left.5 \times 10^{-3} \mathrm{mmol}\right)$, CMA (216 mg, $\left.0.75 \mathrm{mmol}\right)$, ACMAE (84 mg, 0.25 $\mathrm{mmol})$, AIBN $\left(0.16 \mathrm{mg}, 10^{-3} \mathrm{mmol}\right)$ and solvent $(1.66 \mathrm{~g}$, the mass ratio of ethanol/water $=7 / 3$ ) were weighed into a glass tube with a magnetic stir bar. After three freeze-pump-thaw cycles, the tube was sealed, and then polymerization was carried out at $70^{\circ} \mathrm{C}$ for $18 \mathrm{~h}$ under stirring.

RAFT dispersion copolymerization of CMA and comonomer $M$ (DIPEMA, BzMA or ACMAE) to synthesize the $\mathrm{PEO}_{45}-b-\mathrm{P}\left(\mathrm{CMA} \mathrm{A}_{0.6}-\mathrm{co}-\mathrm{M}_{0.4}\right)_{\mathrm{y}}$ vesicles with varying DP of the solvophobic block $(80,100,120,150,200)$ but a fixed molar content of CMA in the solvophobic block at $60 \%$.

The dispersion copolymerization of CMA and comonomer $M$ (DIPEMA, BzMA or ACMAE) to synthesize the $\mathrm{PEO}_{45}-b-\mathrm{P}\left(\mathrm{CMA}_{0.6}-\mathrm{Co}-\mathrm{M}_{0.4)}\right)_{\mathrm{y}}$ vesicles with varying DP of the solvophobic block $(80,100,120,150,200)$ was carried out in 
the ethanol/water mixture $(70 / 30, \mathrm{w} / \mathrm{w})$ at $70^{\circ} \mathrm{C}$ at a fixed chain concentration of $3.01 \times 10^{-3} \mathrm{mmol} / \mathrm{g}$. The details in feeds for the fabrication of the PEO ${ }_{45}-b-\mathrm{P}\left(\mathrm{CMA}_{0.6}-\mathrm{Co}-\mathrm{M}_{0.4}\right)_{\mathrm{y}}$ vesicles are summarized in Table S9. Herein, a typical copolymerization of CMA and ACMAE to fabricate the

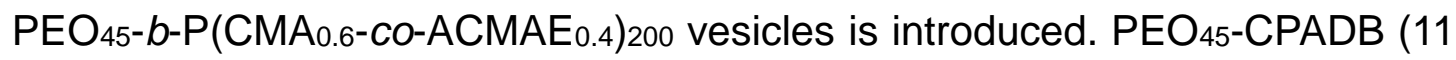
$\left.\mathrm{mg}, 5 \times 10^{-3} \mathrm{mmol}\right)$, CMA (173 mg, $\left.0.6 \mathrm{mmol}\right)$, ACMAE (134 mg, $\left.0.4 \mathrm{mmol}\right)$, AIBN $\left(0.16 \mathrm{mg}, 10^{-3} \mathrm{mmol}\right)$ and solvent $(1.66 \mathrm{~g}$, the mass ratio of ethanol/water $=7 / 3$ ) were weighed into a glass tube with a magnetic stir bar. After three freeze-pump-thaw cycles, the tube was sealed, and then polymerization was carried out at $70^{\circ} \mathrm{C}$ for $18 \mathrm{~h}$ under stirring.

\section{Characterization.}

The ${ }^{1} \mathrm{H}$ NMR $(400 \mathrm{MHz})$ spectra were obtained on a Bruker DMX300 spectrometer in $\mathrm{CDCl}_{3}$ with tetra-methylsilane as an internal reference. For the samples shown in Figure $\mathrm{S} 1$ and $\mathrm{S} 8$, Waters 515 gel permeation chromatography (GPC) equipped with two ultrastyragel columns in series and RI 2414 detector was utilized to measure the molecular weight $\left(M_{n}\right)$ and $M_{w} / M_{n}$, and DMF was used as eluent with a flow rate of $1.0 \mathrm{~mL} / \mathrm{min}$. For other samples, Waters 1515 gel permeation chromatography (GPC) equipped with three ultrastyragel columns in series and RI 2414 detector was utilized to measure the molecular weight $\left(M_{n}\right)$ and $M_{w} / M_{n}$, and THF was used as eluent 
with a flow rate of $1.0 \mathrm{~mL} / \mathrm{min}$. These systems were calibrated with near monodispersed polystyrene standards (Sigma-Aldrich) with molecular weight ranging from $0.5 \times 10^{3} \mathrm{~g} / \mathrm{mol}$ to $6 \times 10^{5} \mathrm{~g} / \mathrm{mol}$. Transmission electron microscope (TEM) imaging was performed on a Hitachi $\mathrm{H}-800$ electron microscope at an accelerating voltage of $100 \mathrm{kV}$. The dispersion of vesicles was diluted with the ethanol/water mixture $(70 / 30, \mathrm{w} / \mathrm{w})$ to $0.5 \% \mathrm{w} / \mathrm{w}$ for TEM observations. $10 \mu \mathrm{L}$ of the diluted dispersion solution was deposited on copper grid which coated with thin films of formvar and carbon successively. The dynamic light scattering (DLS) measurements were conducted on a commercial Laser Light Scattering spectrometer (Zetasizer Nano ZS90, Malven Instruments Ltd., Malvern, UK) equipped with a He-Ne Laser (4.0 mW, $633 \mathrm{~nm}$ ) at $25^{\circ} \mathrm{C}$ and a fixed angle of $90^{\circ}$, and all the data were averaged over three times of measurements. Fluorescence emission spectra was obtained using F-4600 FL Spectrophotometer with excitation wavelength at $320 \mathrm{~nm}$, scanning from $330-600 \mathrm{~nm}$ at $1200 \mathrm{~nm} / \mathrm{min}$, setting an excitation slit width and an emission slit width both at $5.0 \mathrm{~nm}$. Ultra-sensitive differential scanning calorimetry (US-DSC) analysis was performed on a MicroCal VP-DSC differential scanning calorimeter with a heating rate of $1{ }^{\circ} \mathrm{C} \min ^{-1}$ using ethanol/water $(7 / 3, \mathrm{w} / \mathrm{w})$ as the reference. 


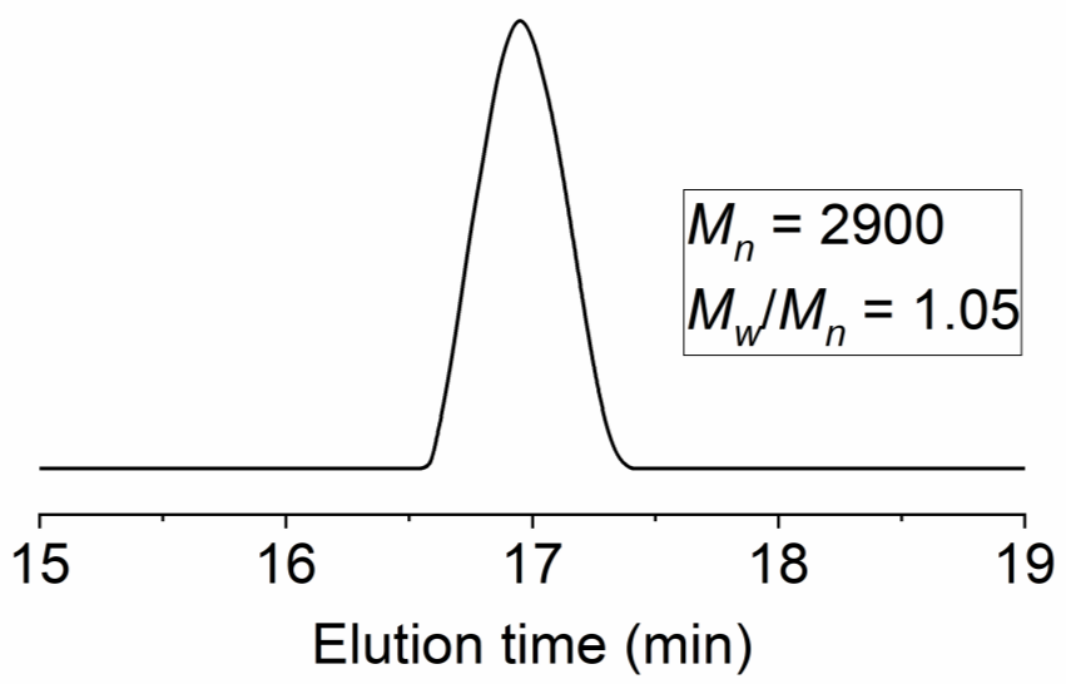

Fig. S1 GPC trace of PEO45-CPADB. 

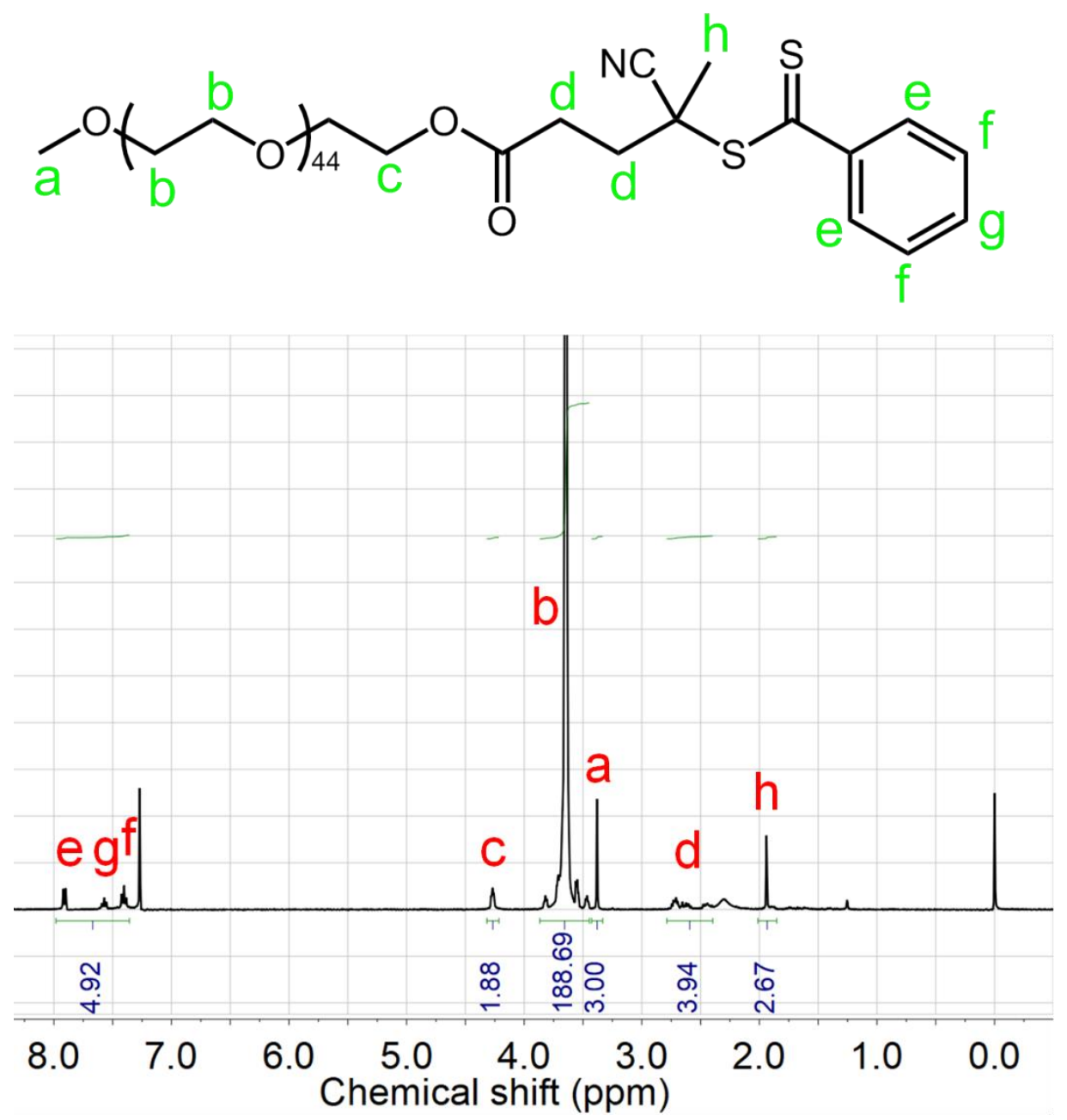

Fig. $52{ }^{1} \mathrm{H}$ NMR spectrum of $\mathrm{PEO}_{45}-\mathrm{CPADB}$ in $\mathrm{CDCl}_{3}$. End-functionality of CPADB in PEO45-CPADB $\left(F_{C P A D B}\right)$ is calculated according to the following equation S1.

$$
F_{C P A D B}=\frac{\left(I_{e, g, f}+I_{c}\right) \times \frac{1}{7}}{I_{a} \times \frac{1}{3}} \times 100 \% \approx 97.1 \%
$$



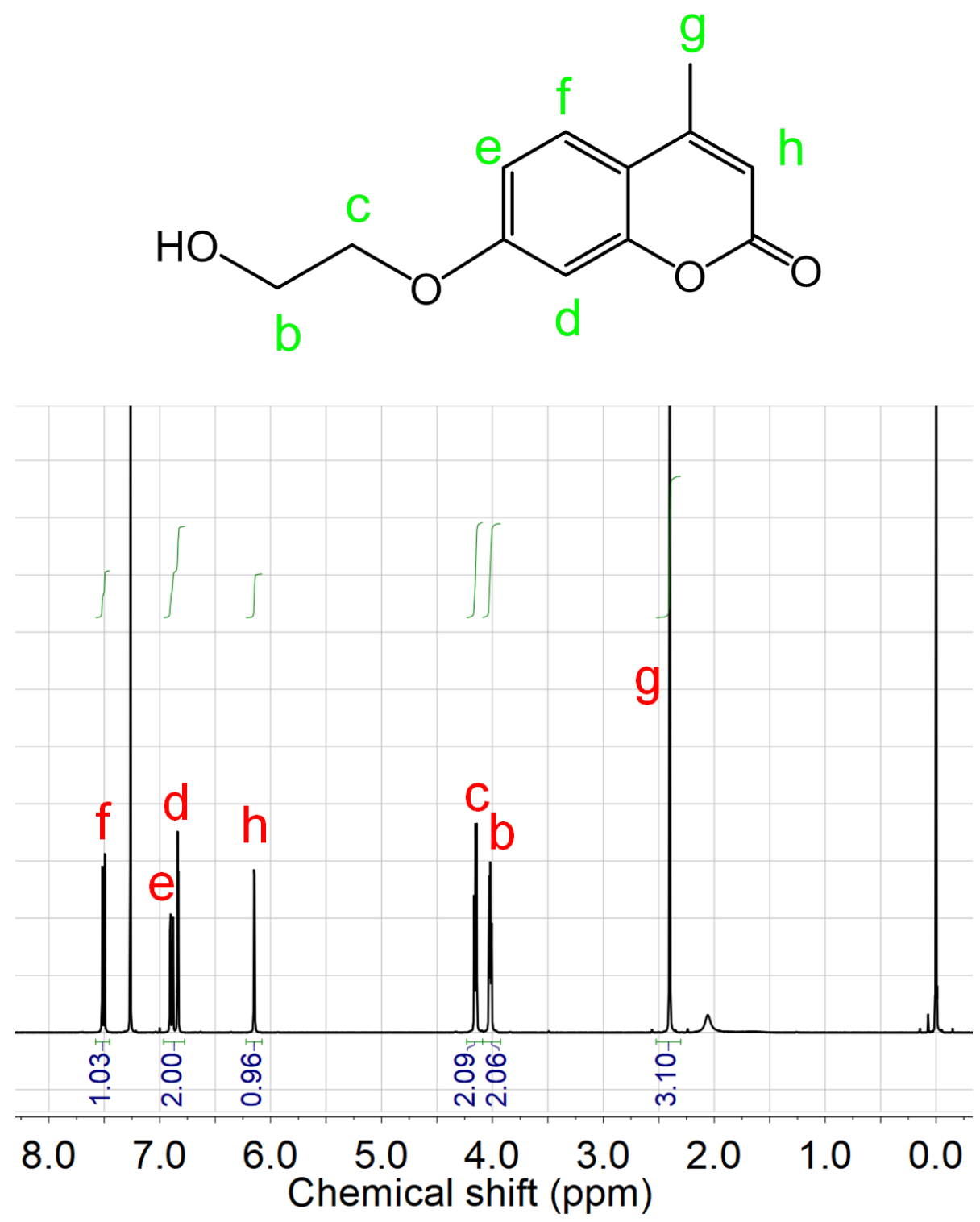

Fig. S3 ${ }^{1} \mathrm{H}$ NMR spectrum of 7-(2-hydroxyethoxy)-4-methylcoumarin in $\mathrm{CDCl}_{3}$. 

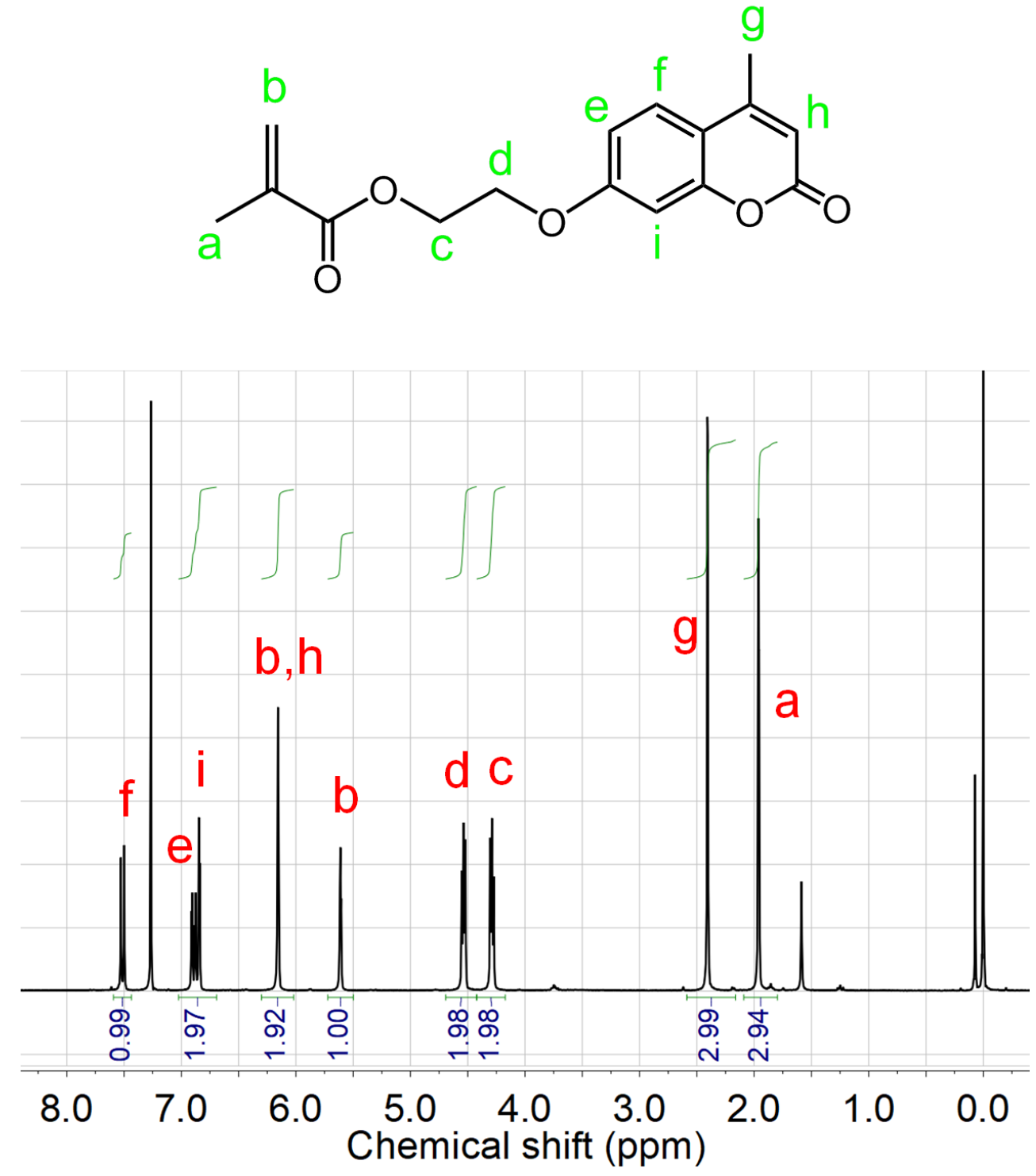

Fig. S4 ${ }^{1} \mathrm{H}$ NMR spectrum of 7 -(2-methacryloyloxyethoxy)- 4-methylcoumarin $(\mathrm{CMA})$ in $\mathrm{CDCl}_{3}$. 


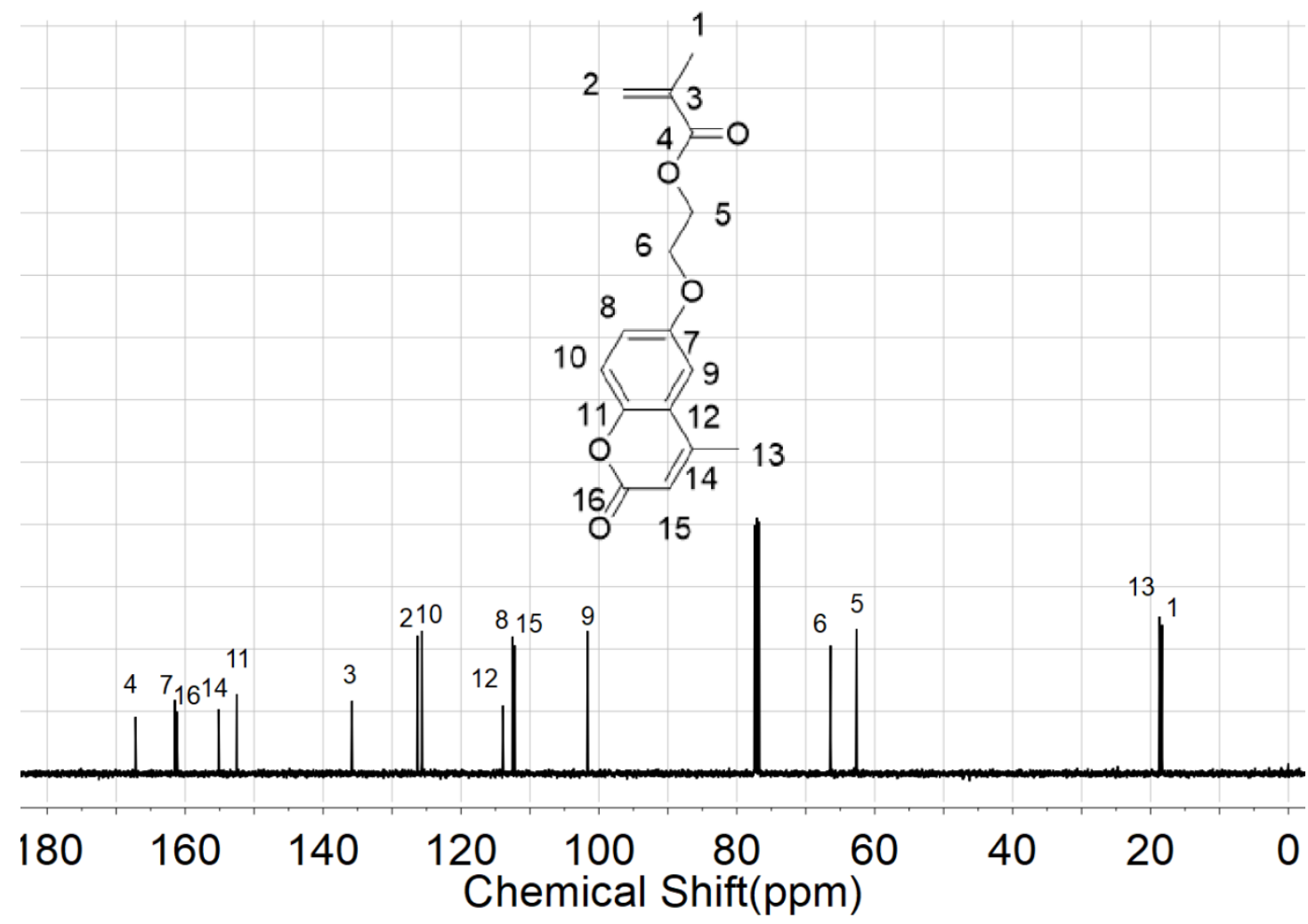

Fig. S5 ${ }^{13} \mathrm{C}$ NMR spectrum of 7-(2-methacryloyloxyethoxy)- 4-methylcoumarin $(\mathrm{CMA})$ in $\mathrm{CDCl}_{3}$. 

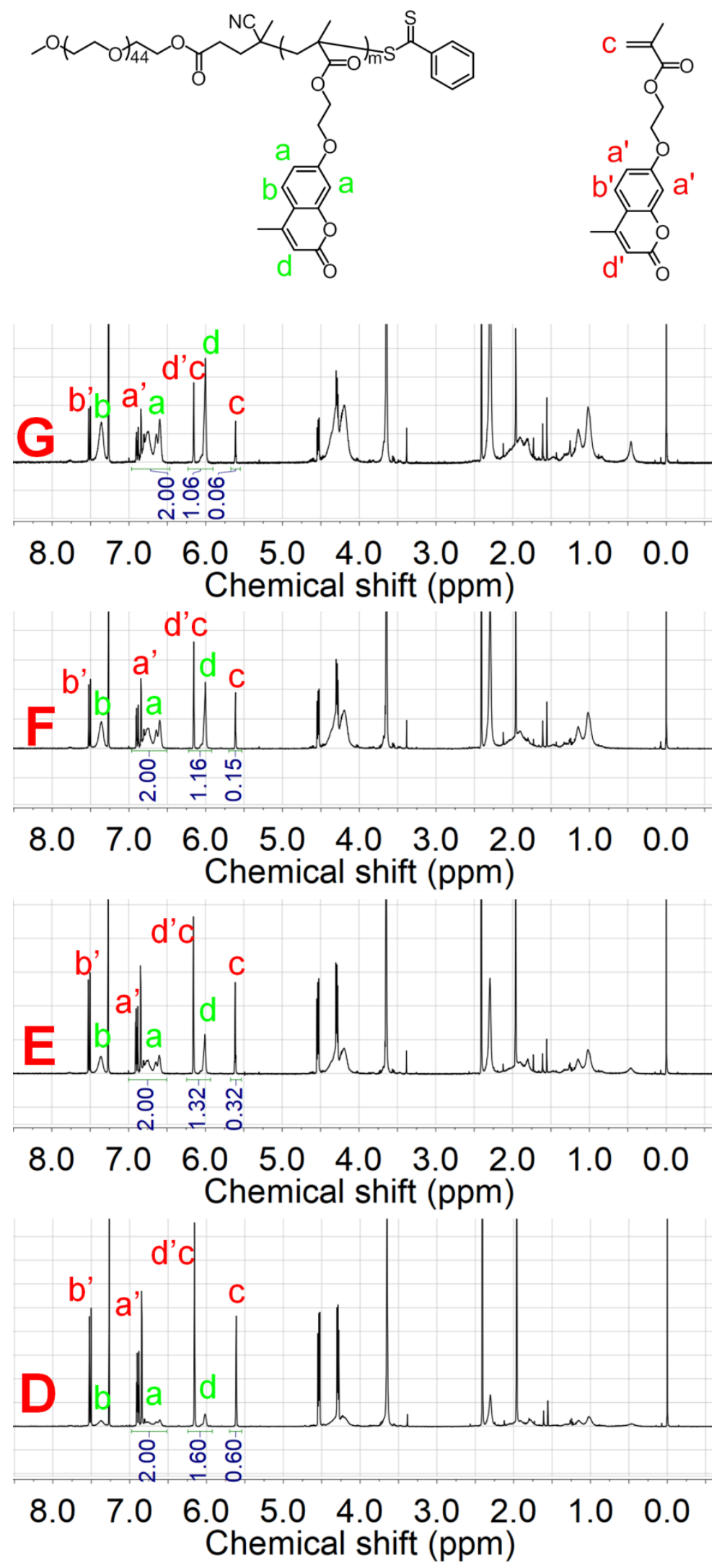

(continue on the next page) 

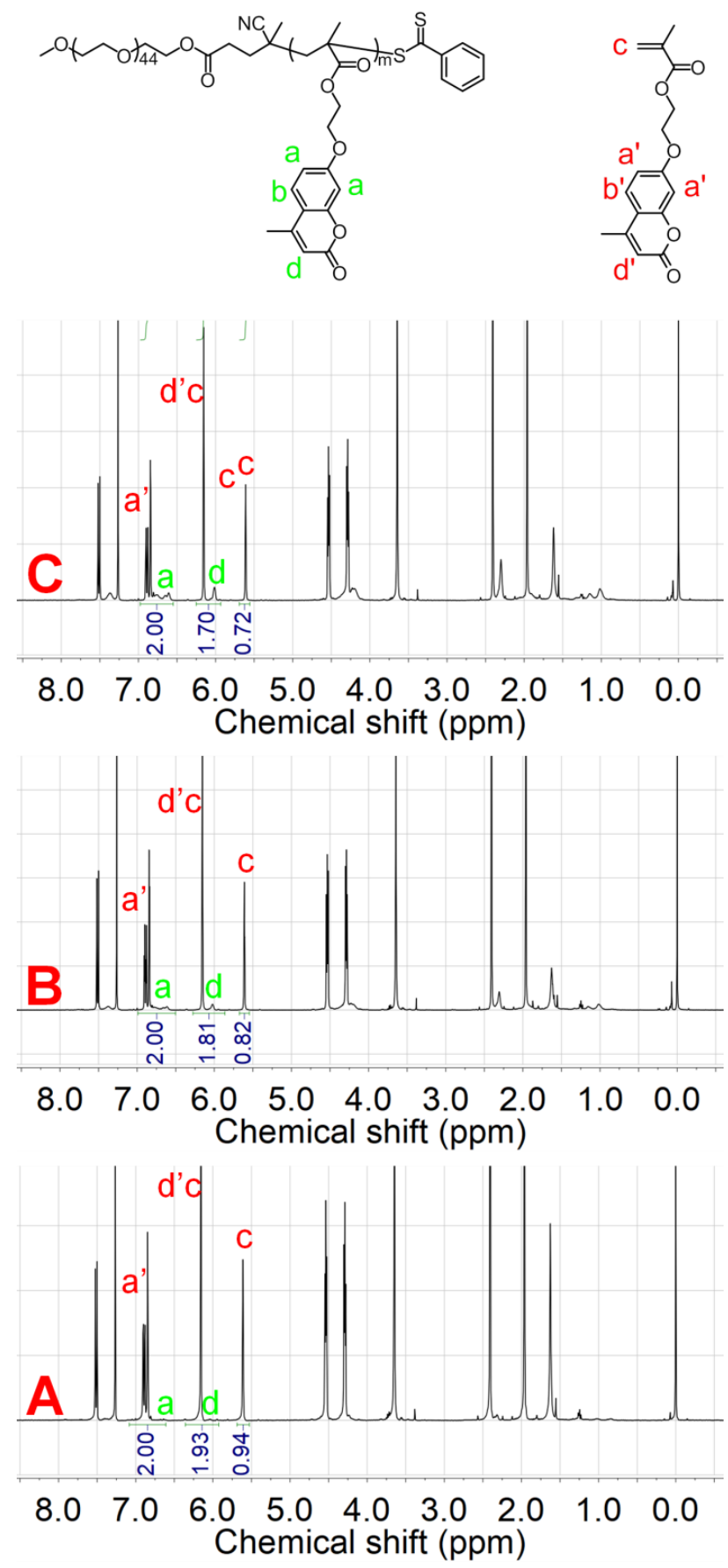

Fig. S6 ${ }^{1} \mathrm{H}$ NMR spectra of the polymerization mixture at different polymerization time, (A) 0.75 h, (B) $1.25 \mathrm{~h},(C) 1.5 \mathrm{~h},(D) 2 \mathrm{~h},(\mathrm{E}) 3 \mathrm{~h},(\mathrm{~F}) 4 \mathrm{~h},(\mathrm{G}) 6 \mathrm{~h}$. For each sample, $50 \mu \mathrm{L}$ of the polymerization mixture was taken out at different polymerization time, naturally dried (the residual CMA will not reduce during this procedure), and then added into $0.6 \mathrm{~mL} \mathrm{CDCl}_{3}$ for ${ }^{1} \mathrm{H}$ NMR characterization. The conversions of CMA at different polymerization time were calculated according to equation S2. The conversion of CMA is defined as the ratio of CMA that converted into polymer to the overall applied CMA (including reacted and unreacted CMA).

$$
\text { Conversion }_{C M A}(\%)=1-\frac{2 I_{C}}{I_{a^{+}+a_{a}^{\prime}}} \times 100 \% \quad \text { Equation S2 }
$$




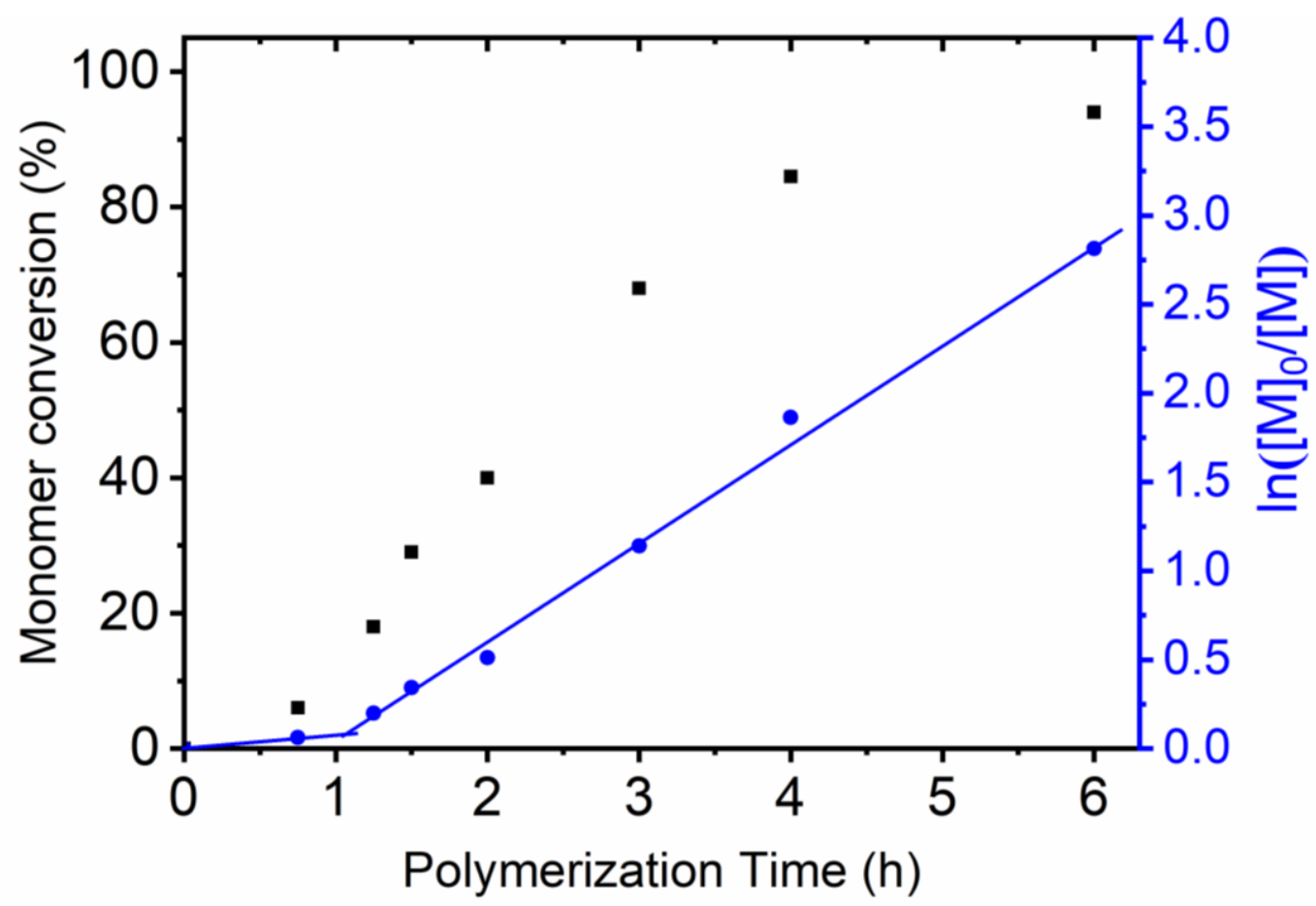

Fig. S7 Polymerization kinetics for the RAFT dispersion polymerization of CMA using PEO-CPADB as the macro-RAFT agent. 


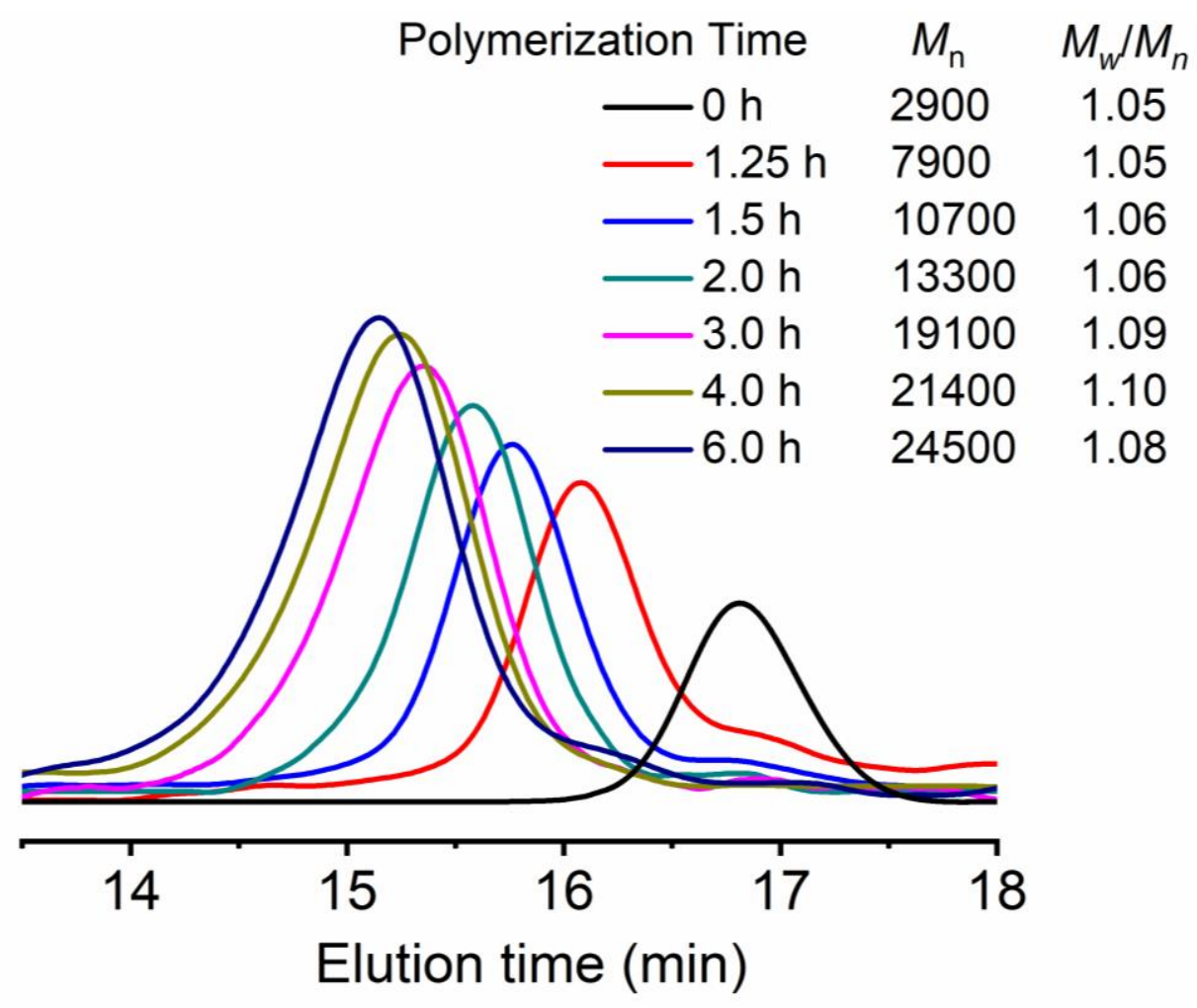

Fig. S8 GPC traces of the block copolymers PEO-b-PCMA obtained at different polymerization time. 

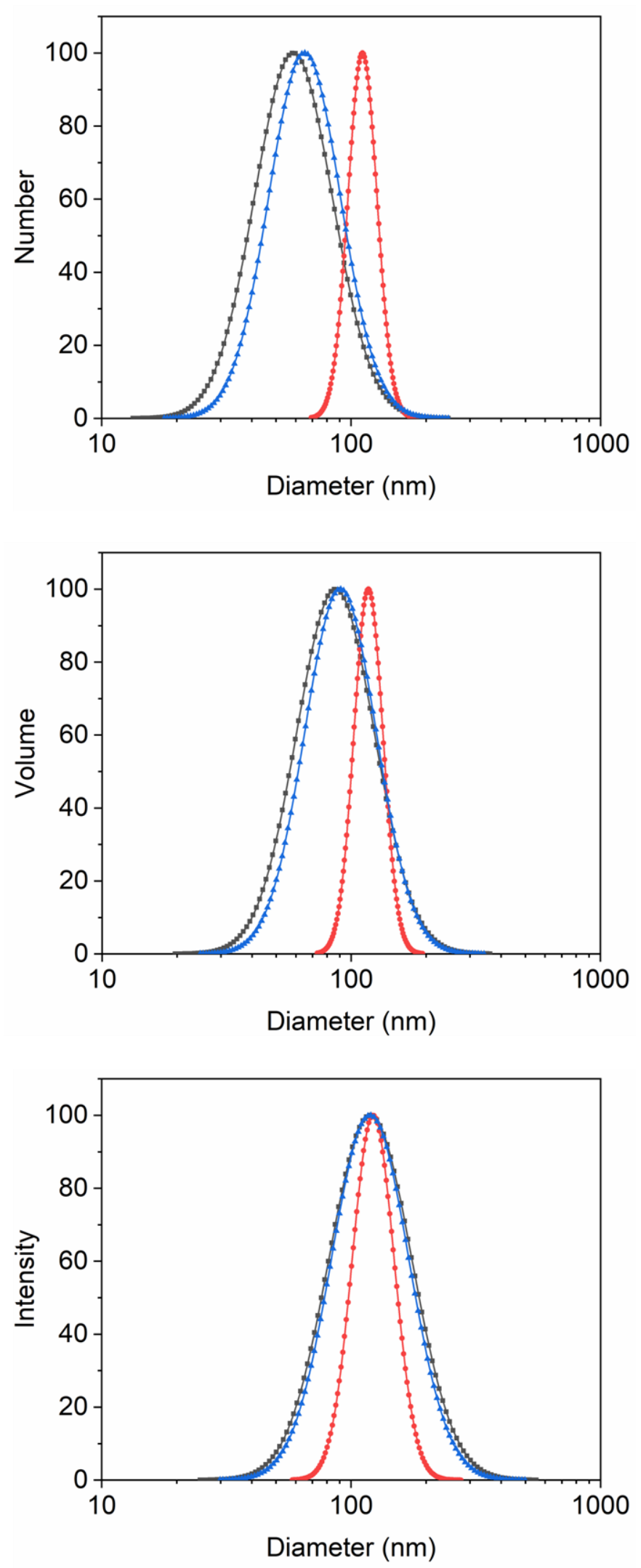

Fig. 59 DLS results of the $\mathrm{PEO}_{45}-b-\mathrm{PCMA}_{80}$ vesicles. 
<smiles></smiles>

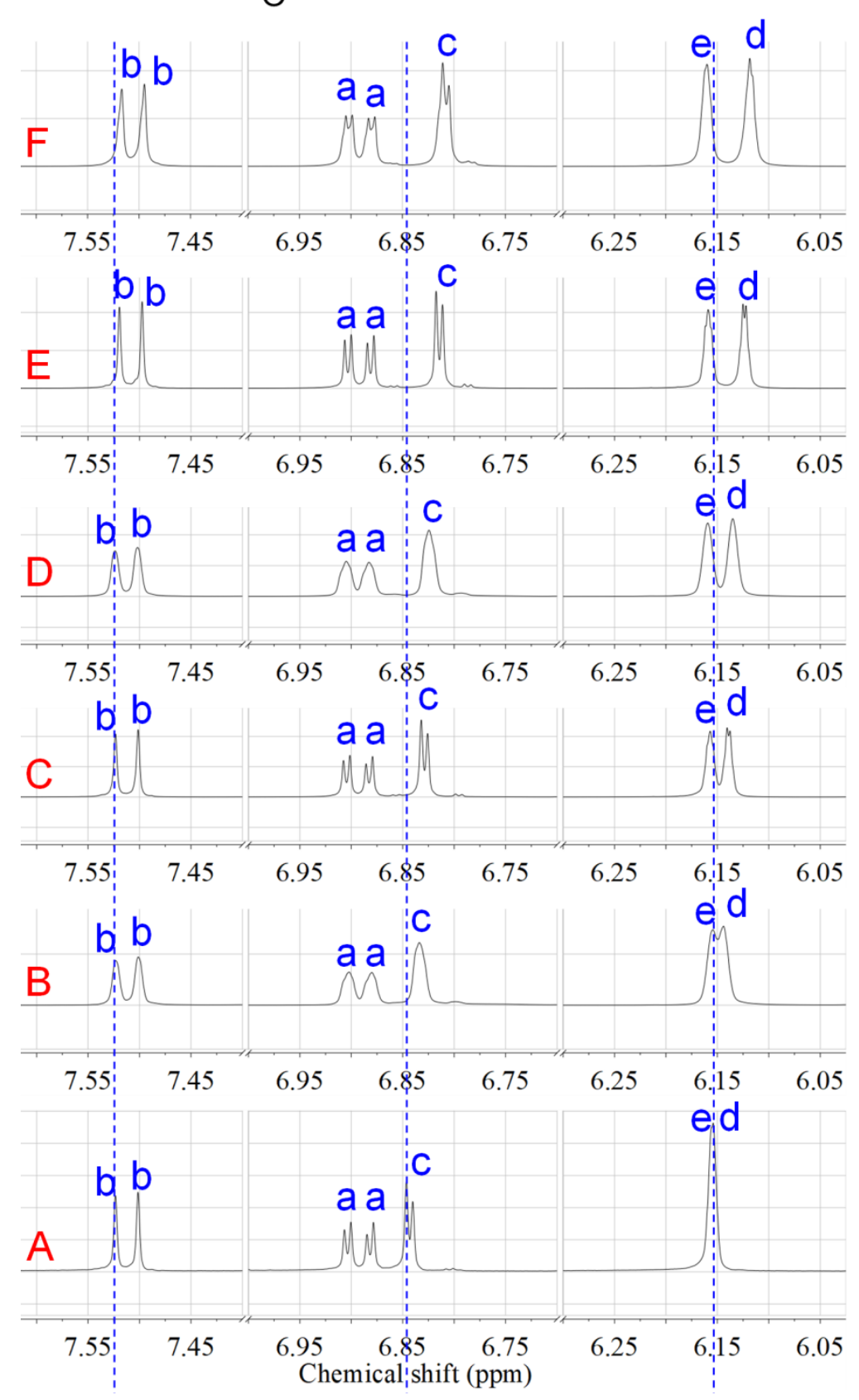

Fig. $\mathrm{S} 10{ }^{1} \mathrm{H}$ NMR spectra of $\mathrm{CMA}$ in $\mathrm{CDCl}_{3}$ at different concentrations, (A) 5 $\mathrm{mg} / \mathrm{mL}$, (B) $50 \mathrm{mg} / \mathrm{mL}$, (C) $75 \mathrm{mg} / \mathrm{mL}$, (D) $100 \mathrm{mg} / \mathrm{mL}$, (E) $150 \mathrm{mg} / \mathrm{mL}$, and (F) $200 \mathrm{mg} / \mathrm{mL}$. 

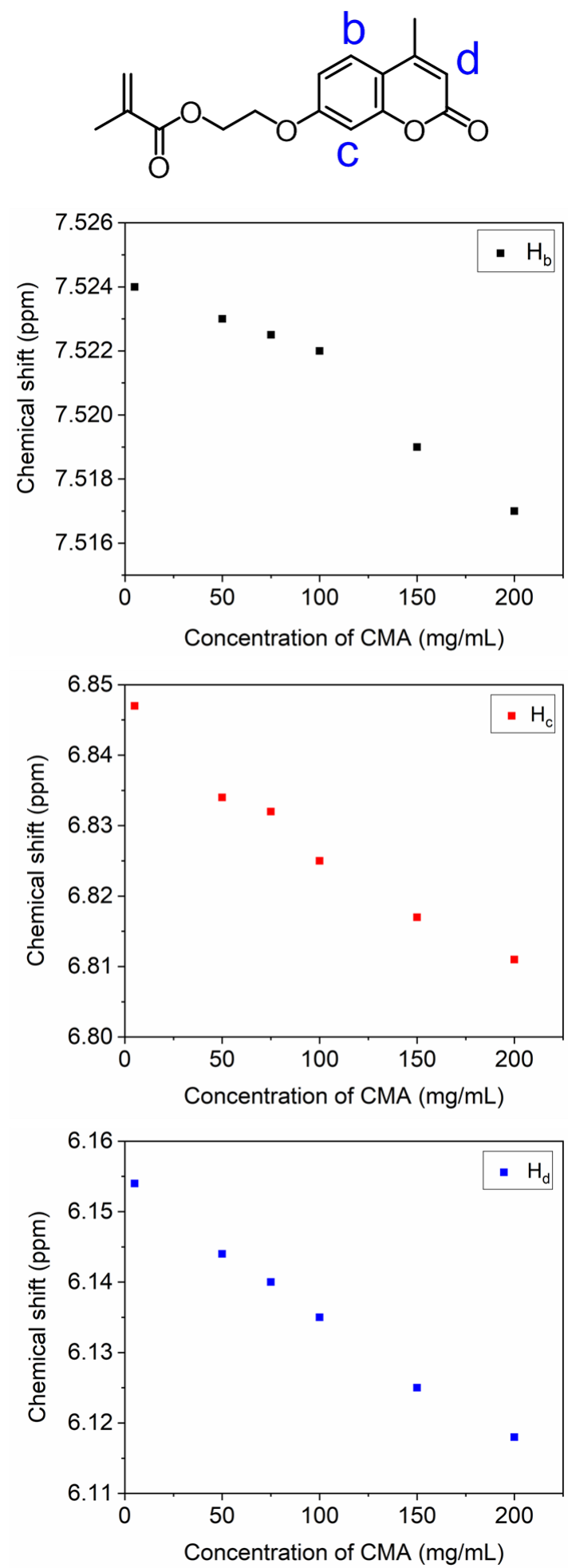

Fig. S11 Chemical shift of protons in coumarin unit at different concentrations of CMA. 


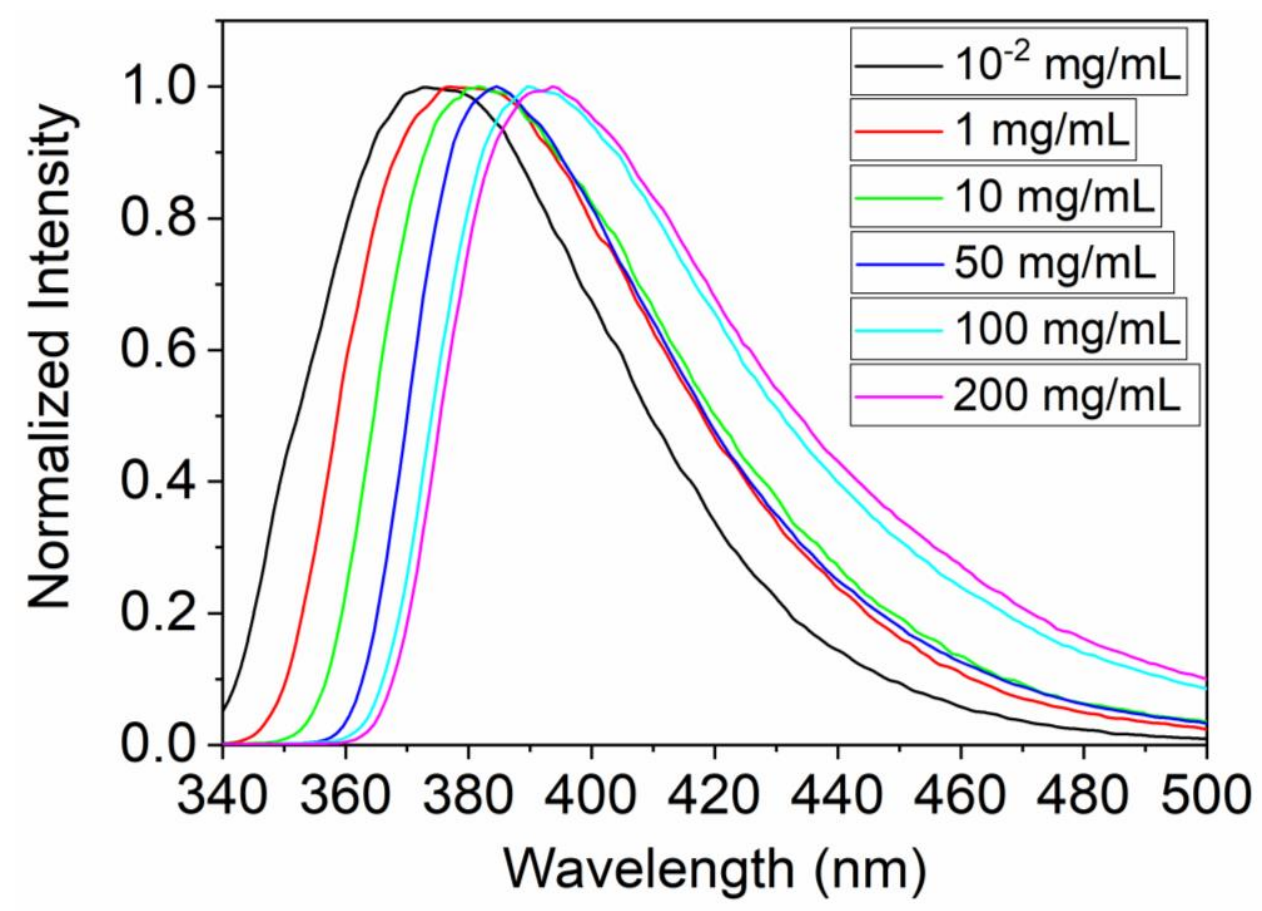

Fig. S12 Normalized fluorescence emission spectra of CMA monomer at different concentration in $\mathrm{CH}_{2} \mathrm{Cl}_{2}$ with excitation wavelength at $320 \mathrm{~nm}$. 
Table S1. The details in feeds for the fabrication of the PEO $45-b-P\left(C M A_{1-x}-c o-D I P E M A_{x}\right) 80$ vesicles.

\begin{tabular}{|c|c|c|c|c|c|c|}
\hline $\begin{array}{c}{ }^{{ }^{a}} \text { PEO }_{45-b-P}\left(\mathrm{CMA}_{1-\mathrm{x}}\right. \\
\text { co-DIPEMA })_{80}\end{array}$ & PEO $_{45}$-CPADB & CMA & 'DIPEMA & Ethanol/water (7/3) & solid content & ${ }^{c}$ chain concentration \\
\hline$x=0.05$ & $11 \mathrm{mg}\left(5 \times 10^{-3} \mathrm{mmol}\right)$ & $109 \mathrm{mg}(0.38 \mathrm{mmol})$ & $4.3 \mathrm{mg}(0.02 \mathrm{mmol})$ & $2.36 \mathrm{~g}$ & $5 \%$ & $2.12 \times 10^{-3} \mathrm{mmol} / \mathrm{g}$ \\
\hline$x=0.10$ & $11 \mathrm{mg}\left(5 \times 10^{-3} \mathrm{mmol}\right)$ & 104 mg (0.36 mmol) & $8.5 \mathrm{mg}(0.04 \mathrm{mmol})$ & $2.35 \mathrm{~g}$ & $5 \%$ & $2.13 \times 10^{-3} \mathrm{mmol} / \mathrm{g}$ \\
\hline$x=0.15$ & $11 \mathrm{mg}\left(5 \times 10^{-3} \mathrm{mmol}\right)$ & $98 \mathrm{mg}(0.34 \mathrm{mmol})$ & $12.8 \mathrm{mg}(0.06 \mathrm{mmol})$ & $2.31 \mathrm{~g}$ & $5 \%$ & $2.16 \times 10^{-3} \mathrm{mmol} / \mathrm{g}$ \\
\hline$x=0.25$ & $11 \mathrm{mg}\left(5 \times 10^{-3} \mathrm{mmol}\right)$ & 86 mg (0.30 mmol) & $21.3 \mathrm{mg}(0.10 \mathrm{mmol})$ & $2.25 \mathrm{~g}$ & $5 \%$ & $2.22 \times 10^{-3} \mathrm{mmol} / \mathrm{g}$ \\
\hline$x=0.40$ & $11 \mathrm{mg}\left(5 \times 10^{-3} \mathrm{mmol}\right)$ & $69 \mathrm{mg}$ (0.24 mmol) & $34.1 \mathrm{mg}(0.16 \mathrm{mmol})$ & $2.17 \mathrm{~g}$ & $5 \%$ & $2.30 \times 10^{-3} \mathrm{mmol} / \mathrm{g}$ \\
\hline
\end{tabular}

${ }^{a} x$ is the feed molar ratio of DIPEMA/(CMA+DIPEMA). bIn order to minimize the errors in weighing, the DIPEMA was diluted with ethanol/water (7/3) to

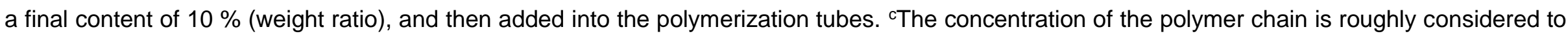
be the same as the concentration of the $\mathrm{PEO}_{45}$-CPADB. 
Table S2. RAFT dispersing copolymerization of CMA and DIPEMA with various molar ratio of DIPEMA/(CMA+DIPEMA) (x) to fabricate a series of $\mathrm{PEO}_{45}-b-\mathrm{P}\left(\mathrm{CMA}_{1-\mathrm{x}}-\text { Co-DIPEMA }\right)_{80}$ vesicles, the monomer conversion of $\mathrm{CMA}$ and DIPEMA, $M_{n}$ and $M_{w} / M_{n}$ of the resultant block copolymer $\mathrm{PEO}_{45}-b-\mathrm{P}\left(\mathrm{CMA}_{1-\mathrm{x}-\mathrm{CO}-\mathrm{DIPEMA}}\right)_{80}$, and intensity-/volume-/number-average diameter of the corresponding vesicles.

\begin{tabular}{|c|c|c|c|c|c|c|c|c|}
\hline $\begin{array}{l}\text { aPEO }_{45-b-}\left(\mathrm{CMA}_{1-\mathrm{x}}-\right. \\
\text { co-DIPEMAX) }\end{array}$ & $\begin{array}{l}{ }^{\mathrm{b} C o n v e r s i o n} \\
\text { of CMA(\%) }\end{array}$ & $\begin{array}{l}\text { bConversion of } \\
\text { DIPEMA(\%) }\end{array}$ & $M_{n}$ & $M_{w} / M_{n}$ & $\begin{array}{l}\text { 'Intensity-average } \\
\text { diameter }(\mathrm{nm})\end{array}$ & $\begin{array}{l}\text { 'Volume-average } \\
\text { diameter }(\mathrm{nm})\end{array}$ & $\begin{array}{l}\text { 'Number-average } \\
\text { diameter }(\mathrm{nm})\end{array}$ & 'PDI \\
\hline$x=0.05$ & 97 & 82 & 25700 & 1.32 & $130.5 \pm 4.9$ & $108.4 \pm 10.6$ & $83.9 \pm 31.1$ & $0.11 \pm 0.03$ \\
\hline$x=0.10$ & 98 & 83 & 25900 & 1.27 & $179.9 \pm 25.8$ & $139.4 \pm 40.5$ & $76.8 \pm 42.5$ & $0.13 \pm 0.02$ \\
\hline$x=0.15$ & 95 & 89 & 26700 & 1.28 & $235.1 \pm 12.4$ & $172.7 \pm 25.8$ & $68.1 \pm 4.2$ & $0.18 \pm 0.04$ \\
\hline$x=0.25$ & 91 & 82 & 27000 & 1.27 & $516.0 \pm 43.8$ & $615.5 \pm 260.0$ & $186.3 \pm 75.5$ & $0.29 \pm 0.01$ \\
\hline$x=0.40$ & 89 & 85 & 24100 & 1.23 & $1061.2 \pm 632.0$ & $722.8 \pm 120.2$ & $333.5 \pm 158.9$ & $0.26 \pm 0.01$ \\
\hline
\end{tabular}




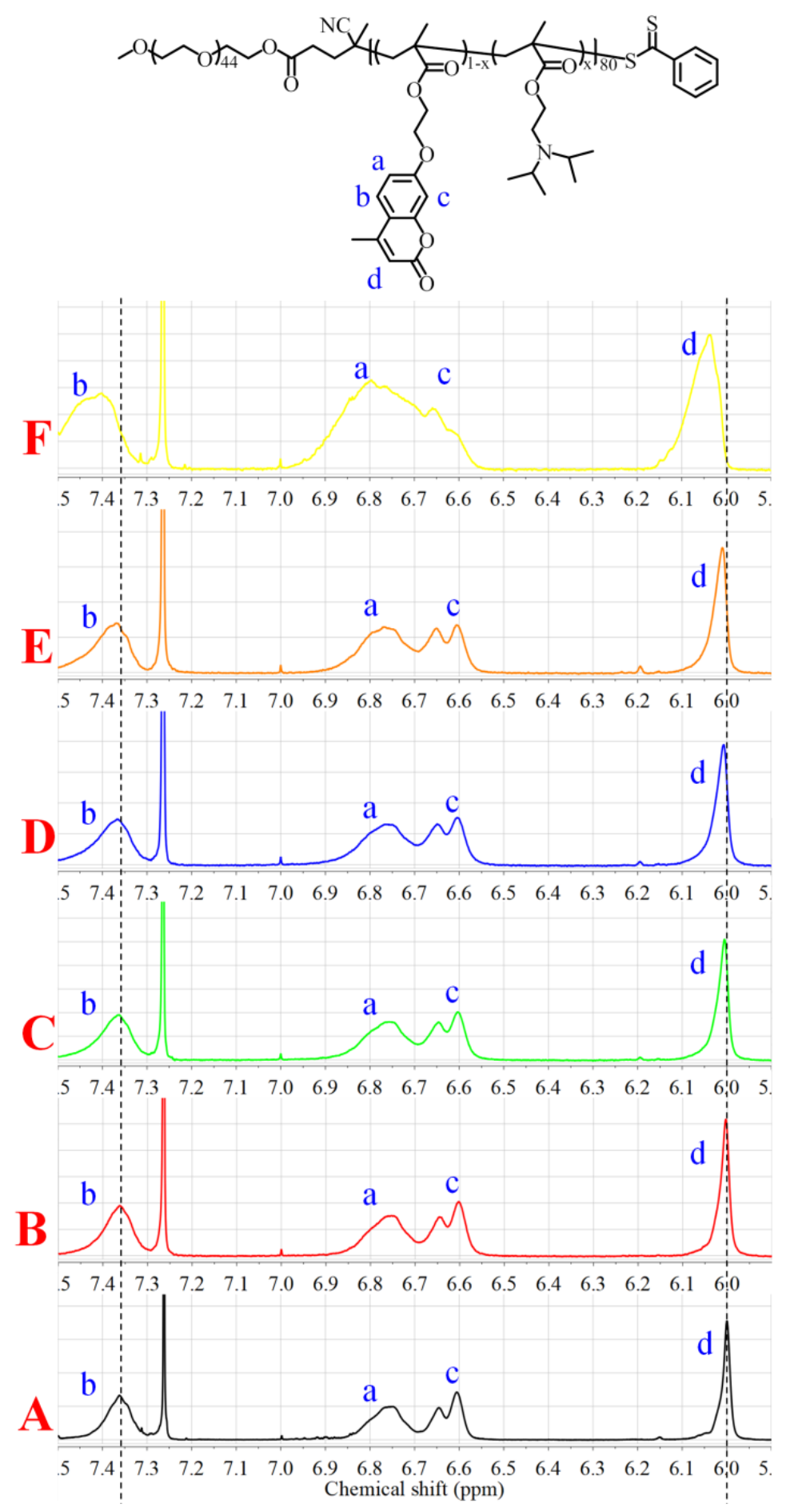

Fig. $\quad \mathrm{S} 13 \quad{ }^{1} \mathrm{H} \quad \mathrm{NMR}$ spectra of diblock terpolymers

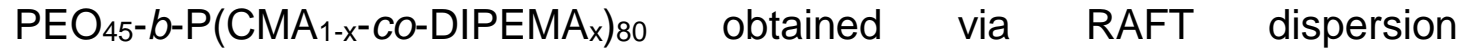
copolymerization of CMA and DIPEMA with different feed molar ratio of DIPEMA/(CMA+DIPEMA), (A) $x=0$, (B) $x=0.05$, (C) $x=0.10$, (D) $x=0.15$, (E) $x=0.25$, and $(F) x=0.40$. The chemical shift $(\delta)$ of $H_{b}$ and $H_{d}$ at $x=0$ were marked by the dash line, which shifted to higher $\delta$ values with increasing of the $x$ values. The chemical shift $(\delta)$ of $H_{b}$ and $H_{d}$ at various $x$ values were summarized in Figure 3C. 

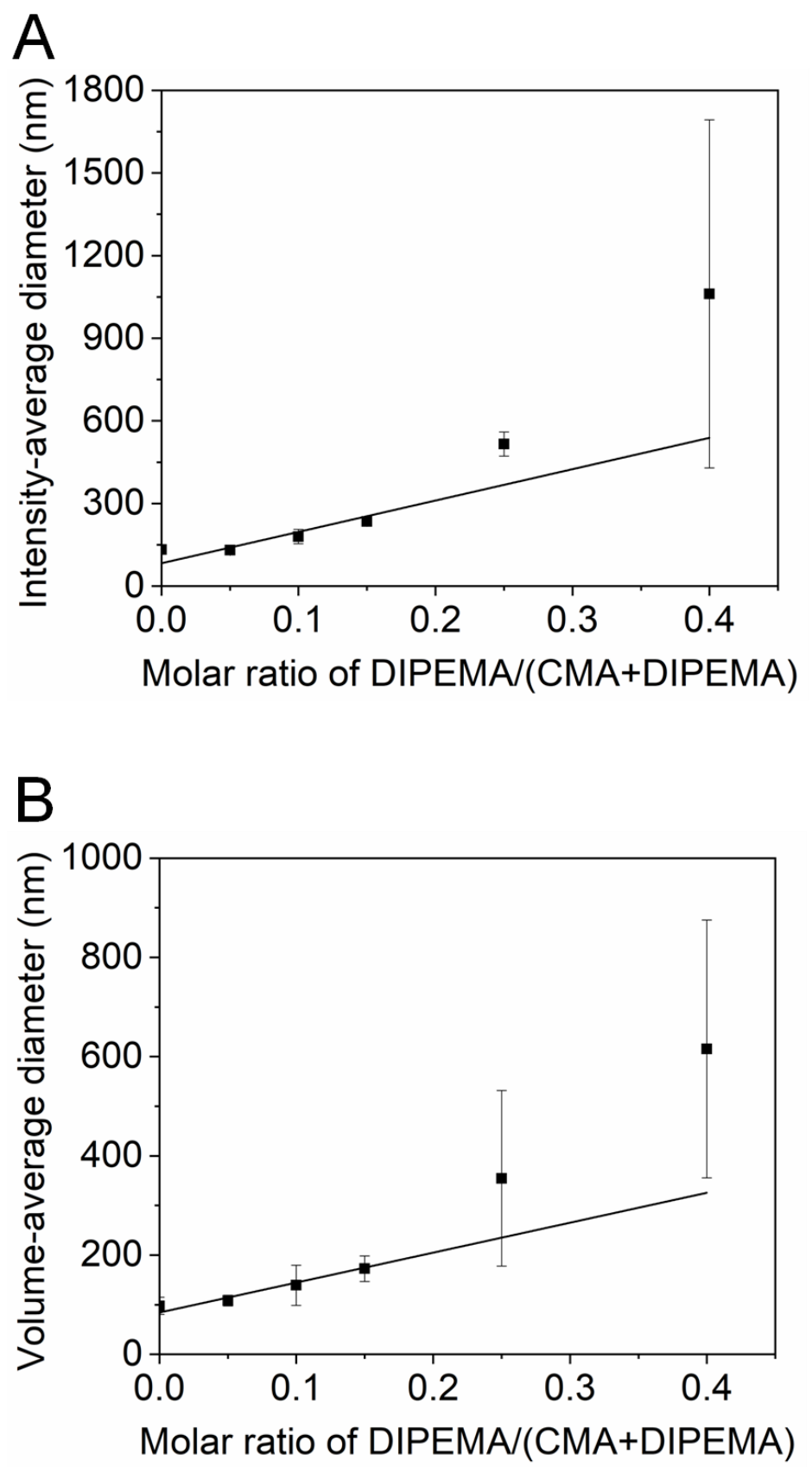

Fig. S14 (A) Intensity-average diameter and (B) volume-average diameter of the $\mathrm{PEO}_{45-b-\mathrm{P}}\left(\mathrm{CMA}_{1-\mathrm{x}-\mathrm{Co}-\mathrm{DIPEMA}}\right)_{80}$ vesicles at different molar ratio of DIPEMA/(CMA+DIPEMA) (x values) obtained via DLS. 


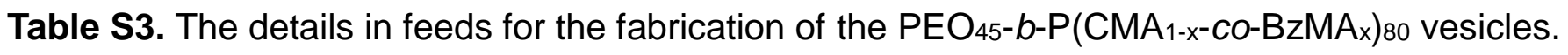

\begin{tabular}{|c|c|c|c|c|c|c|}
\hline $\begin{array}{l}{ }^{{ }^{a} P O_{45}-b-\left(C_{M A} A_{1-x}\right.} \\
\left.c o-B z M A_{x}\right)_{80}\end{array}$ & PEO $_{45}-\mathrm{CPADB}$ & CMA & ${ }^{\mathrm{b}} \mathrm{Bz} M A$ & Ethanol/water $(7 / 3)$ & solid content & ${ }^{\mathrm{c}}$ chain concentration \\
\hline$x=0.05$ & $11 \mathrm{mg}\left(5 \times 10^{-3} \mathrm{mmol}\right)$ & $109 \mathrm{mg}(0.38 \mathrm{mmol})$ & $3.5 \mathrm{mg}(0.02 \mathrm{mmol})$ & $2.35 \mathrm{~g}$ & $5 \%$ & $2.13 \times 10^{-3} \mathrm{mmol} / \mathrm{g}$ \\
\hline$x=0.15$ & $11 \mathrm{mg}\left(5 \times 10^{-3} \mathrm{mmol}\right)$ & $98 \mathrm{mg}(0.34 \mathrm{mmol})$ & $10.6 \mathrm{mg}(0.06 \mathrm{mmol})$ & $2.27 \mathrm{~g}$ & $5 \%$ & $2.20 \times 10^{-3} \mathrm{mmol} / \mathrm{g}$ \\
\hline$x=0.40$ & $11 \mathrm{mg}\left(5 \times 10^{-3} \mathrm{mmol}\right)$ & $69 \mathrm{mg}(0.24 \mathrm{mmol})$ & $28.2 \mathrm{mg}(0.16 \mathrm{mmol})$ & $2.06 \mathrm{~g}$ & $5 \%$ & $2.43 \times 10^{-3} \mathrm{mmol} / \mathrm{g}$ \\
\hline
\end{tabular}


Table S4. RAFT dispersing copolymerization of CMA and BzMA with various molar ratio of BzMA/(CMA+BzMA) ( $x$ ) to fabricate a

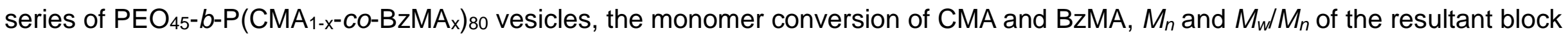
copolymer $\mathrm{PEO}_{45}-b-\mathrm{P}\left(\mathrm{CMA}_{1-\mathrm{x}}-\mathrm{Co}-\mathrm{BzMA}_{\mathrm{x}}\right)_{80}$, and intensity-/volume-/number-average diameter of the corresponding vesicles.

\begin{tabular}{|c|c|c|c|c|c|c|c|c|}
\hline 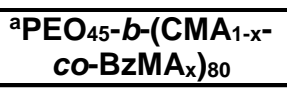 & $\begin{array}{c}{ }^{\mathrm{b}} \text { Conversion } \\
\text { of CMA }(\%)\end{array}$ & $\begin{array}{l}{ }^{\mathrm{b}} \text { Conversion } \\
\text { of } \mathrm{BzMA}(\%)\end{array}$ & $M_{n}$ & $M_{w} / M_{n}$ & $\begin{array}{c}\text { Intensity average } \\
\text { diameter (nm) }\end{array}$ & $\begin{array}{c}\text { Colume average } \\
\text { diameter }(\mathrm{nm})\end{array}$ & $\begin{array}{l}\text { Number average } \\
\text { diameter }(\mathrm{nm})\end{array}$ & 'PDI \\
\hline$x=0.05$ & 95 & 91 & 26400 & 1.35 & $130.1 \pm 3.9$ & $118.4 \pm 19.0$ & $108.7 \pm 32.2$ & $0.08 \pm 0.05$ \\
\hline$x=0.10$ & 93 & 82 & 25800 & 1.40 & $143.6 \pm 6.6$ & $125.6 \pm 30.5$ & $99.7 \pm 39.2$ & $0.11 \pm 0.03$ \\
\hline$x=0.15$ & 93 & 83 & 27200 & 1.38 & $158.5 \pm 18.6$ & $141.0 \pm 34.5$ & $108.2 \pm 26.5$ & $0.13 \pm 0.02$ \\
\hline$x=0.25$ & 95 & 91 & 28500 & 1.28 & $283.8 \pm 29.0$ & $332.6 \pm 51.1$ & $111.6 \pm 26.5$ & $0.25 \pm 0.01$ \\
\hline$x=0.40$ & 96 & 96 & 26500 & 1.25 & $715.9 \pm 194.5$ & $813.0 \pm 231.6$ & $170.7 \pm 32.2$ & $0.28 \pm 0.03$ \\
\hline
\end{tabular}



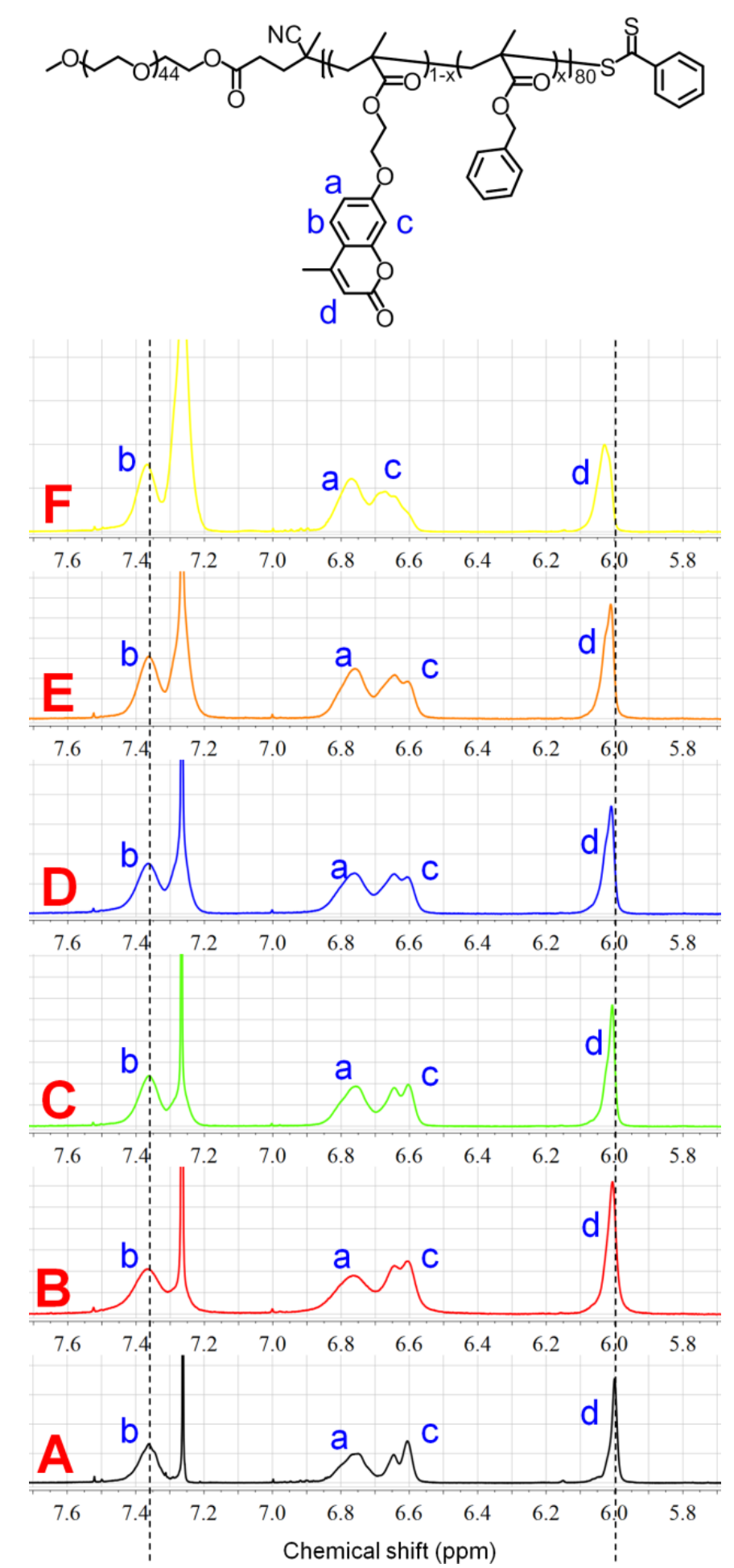

Fig. $\quad \mathrm{S} 15 \quad{ }^{1} \mathrm{H} \quad \mathrm{NMR}$ spectra of diblock terpolymers $\mathrm{PEO}_{45}-b-\mathrm{P}\left(\mathrm{CMA}_{1-x-\mathrm{C}} \mathrm{Co}-\mathrm{BzMA}_{\mathrm{x}}\right)_{80}$ obtained via RAFT dispersion copolymerization of CMA and BzMA with different feed molar ratio of BzMA/(CMA+BzMA), (A) $x=0$, (B) $x=0.05,(C) x=0.10,(D) x=0.15,(E) x=$ 0.25 , and $(F) x=0.40$. The chemical shift $(\delta)$ of $H_{b}$ and $H_{d}$ at $x=0$ were marked by the dash line. The chemical shift $(\delta)$ of $\mathrm{Hb}_{b}$ and $\mathrm{H}_{d}$ at various $x$ values were summarized in Figure 4C. 

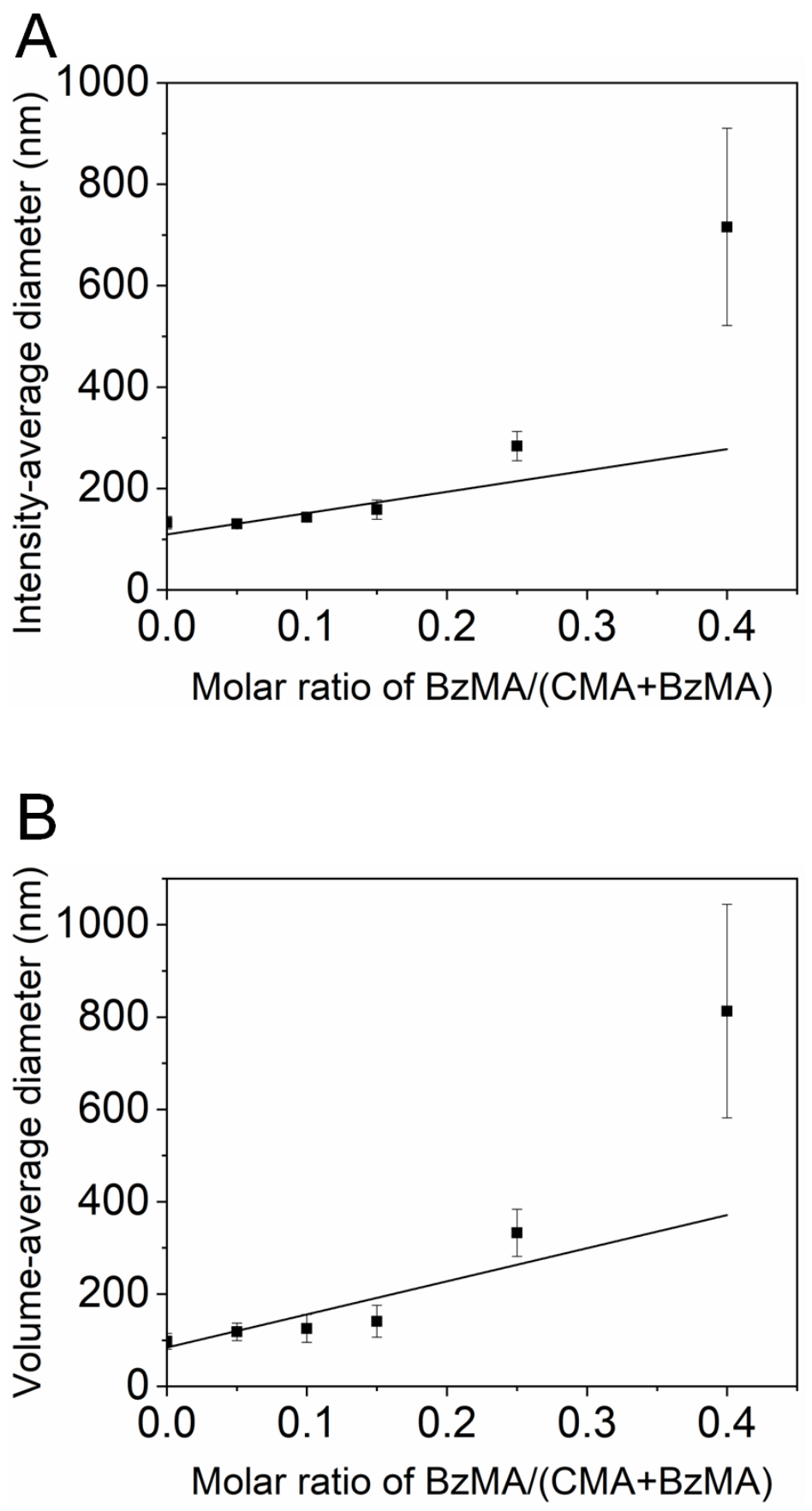

Fig. S16 (A) Intensity-average diameter and (B) volume-average diameter of

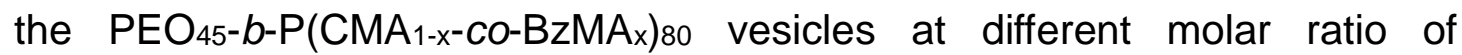
BzMA/(CMA+BzMA) ( $x$ values) obtained via DLS. 
<smiles></smiles>

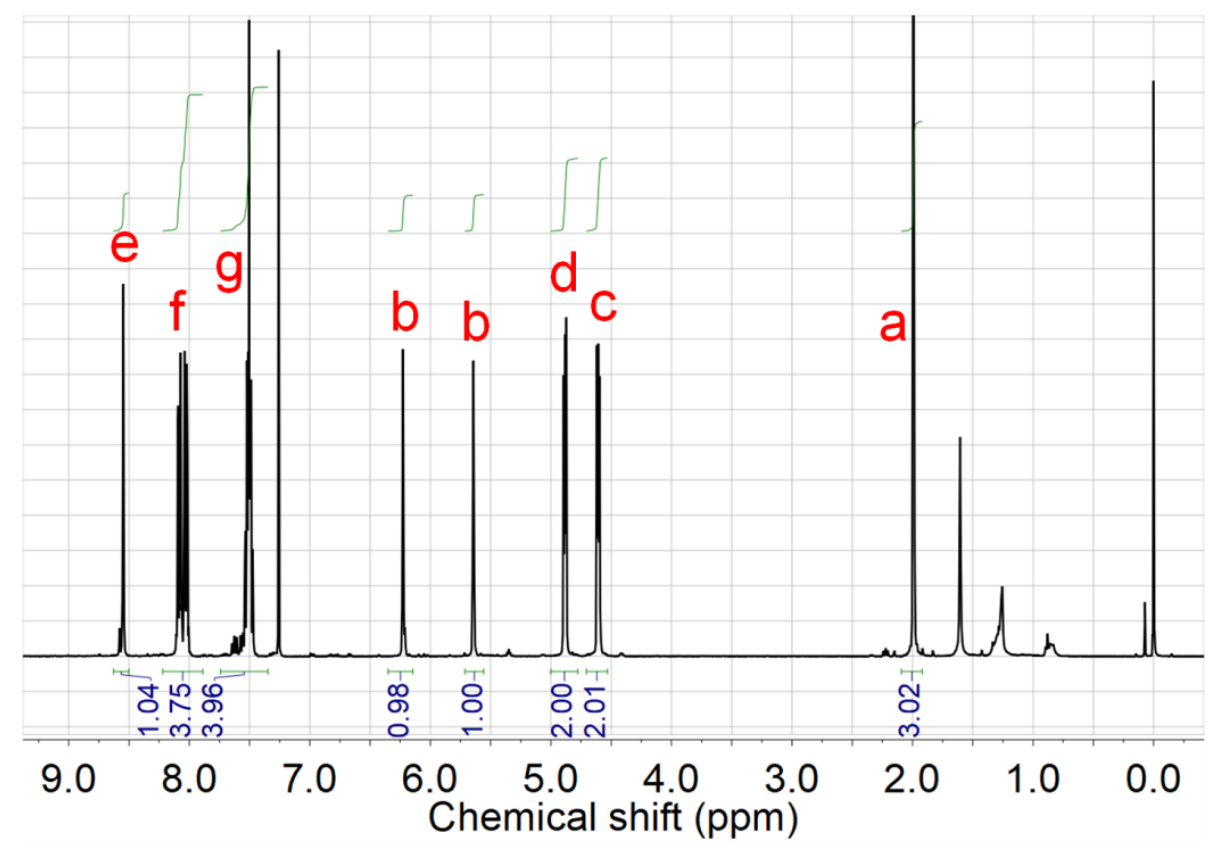

Fig. $\quad$ S17 ${ }^{1} \mathrm{H} \quad$ NMR $\quad$ spectrum of $\quad$-(methacryloyloxy)ethyl anthracene-9-carboxylate (ACMAE) in $\mathrm{CDCl}_{3}$. 

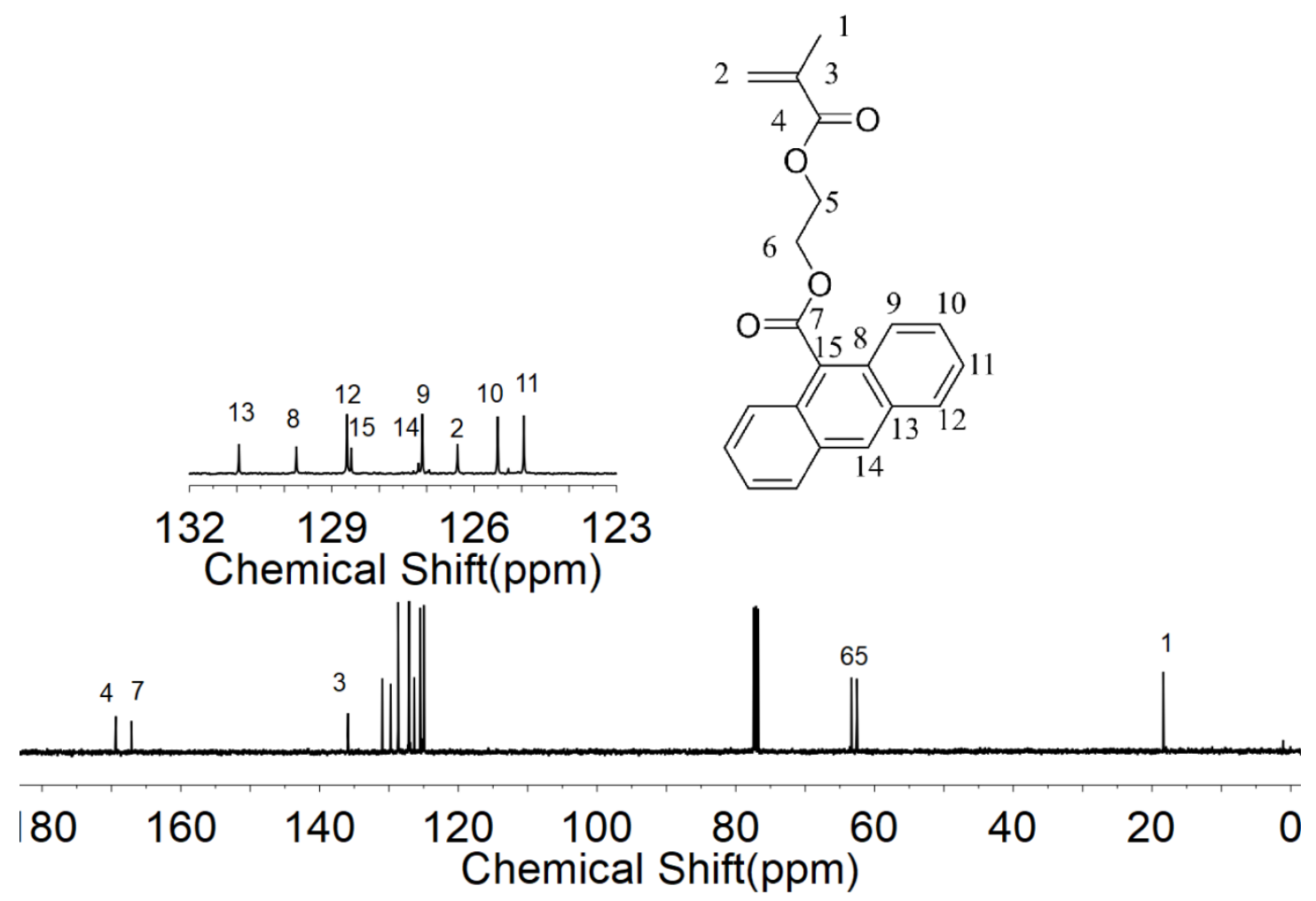

Fig. S18 ${ }^{13} \mathrm{C} \quad$ NMR spectrum of 2-(methacryloyloxy)ethyl anthracene-9-carboxylate (ACMAE) in $\mathrm{CDCl}_{3}$. 


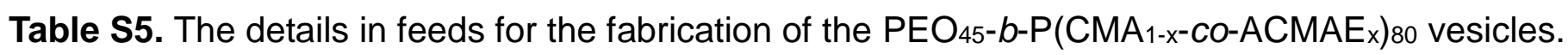

\begin{tabular}{|c|c|c|c|c|c|c|}
\hline $\begin{array}{c}\text { aPEO }_{45-b}-\left(\mathrm{CMA}_{1-\mathrm{x}^{-}}\right. \\
\text {co-ACMAE }{ })_{80}\end{array}$ & $\mathrm{PEO}_{45}-\mathrm{CPADB}$ & CMA & ACMAE & Ethanol/water $(7 / 3)$ & solid content & ${ }^{\mathrm{b}}$ chain concentration \\
\hline$x=0.05$ & $11 \mathrm{mg}\left(5 \times 10^{-3} \mathrm{mmol}\right)$ & $109 \mathrm{mg}(0.38 \mathrm{mmol})$ & 7 mg (0.02 mmol) & $2.41 \mathrm{~g}$ & $5 \%$ & $2.07 \times 10^{-3} \mathrm{mmol} / \mathrm{g}$ \\
\hline$x=0.10$ & $11 \mathrm{mg}\left(5 \times 10^{-3} \mathrm{mmol}\right)$ & $104 \mathrm{mg}(0.36 \mathrm{mmol})$ & $13 \mathrm{mg}(0.04 \mathrm{mmol})$ & $2.43 \mathrm{~g}$ & $5 \%$ & $2.06 \times 10^{-3} \mathrm{mmol} / \mathrm{g}$ \\
\hline$x=0.15$ & $11 \mathrm{mg}\left(5 \times 10^{-3} \mathrm{mmol}\right)$ & $98 \mathrm{mg}(0.34 \mathrm{mmol})$ & $20 \mathrm{mg}(0.06 \mathrm{mmol})$ & $2.45 \mathrm{~g}$ & $5 \%$ & $2.04 \times 10^{-3} \mathrm{mmol} / \mathrm{g}$ \\
\hline$x=0.25$ & $11 \mathrm{mg}\left(5 \times 10^{-3} \mathrm{mmol}\right)$ & $86 \mathrm{mg}(0.30 \mathrm{mmol})$ & $33 \mathrm{mg}(0.10 \mathrm{mmol})$ & $2.47 \mathrm{~g}$ & $5 \%$ & $2.02 \times 10^{-3} \mathrm{mmol} / \mathrm{g}$ \\
\hline$x=0.40$ & $11 \mathrm{mg}\left(5 \times 10^{-3} \mathrm{mmol}\right)$ & $69 \mathrm{mg}(0.24 \mathrm{mmol})$ & $53 \mathrm{mg}(0.16 \mathrm{mmol})$ & $2.53 \mathrm{~g}$ & $5 \%$ & $1.98 \times 10^{-3} \mathrm{mmol} / \mathrm{g}$ \\
\hline$x=0.60$ & $11 \mathrm{mg}\left(5 \times 10^{-3} \mathrm{mmol}\right)$ & $46 \mathrm{mg}(0.16 \mathrm{mmol})$ & $80 \mathrm{mg}(0.24 \mathrm{mmol})$ & $2.60 \mathrm{~g}$ & $5 \%$ & $1.92 \times 10^{-3} \mathrm{mmol} / \mathrm{g}$ \\
\hline
\end{tabular}

${ }^{a} x$ is the feed molar ratio of $A C M A E /(C M A+A C M A E)$. ${ }^{b}$ The concentration of the polymer chain is roughly considered to be the same as the concentration of the $\mathrm{PEO}_{45}-\mathrm{CPADB}$. 
Table S6. RAFT dispersing copolymerization of CMA and ACMAE with various molar ratio of $A C M A E /(C M A+A C M A E)(x)$ to fabricate

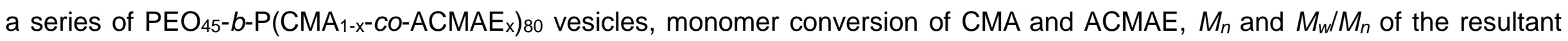
block copolymer $\mathrm{PEO}_{45}-b-\mathrm{P}\left(\mathrm{CMA}_{1-\mathrm{x}}-\mathrm{CO}_{-} \mathrm{ACMAE}\right)_{80}$, and intensity-/volume-/number-average diameter of the corresponding vesicles.

\begin{tabular}{|c|c|c|c|c|c|c|c|c|}
\hline $\begin{array}{c}{ }^{{ }^{2}}{ }^{2} O_{45-}-b-\left(C A_{1-x}-\right. \\
\text { co-ACMAE })_{80}\end{array}$ & $\begin{array}{l}{ }^{\mathrm{b}} \text { Conversion } \\
\text { of CMA }(\%)\end{array}$ & $\begin{array}{c}{ }^{\mathrm{b}} \text { Conversion of } \\
\text { ACMAE }(\%)\end{array}$ & $M_{n}$ & $M_{w} / M_{n}$ & $\begin{array}{c}\text { Intensity average } \\
\text { diameter }(\mathrm{nm})\end{array}$ & $\begin{array}{c}{ }^{\mathrm{C}} \text { Volume average } \\
\text { diameter }(\mathrm{nm})\end{array}$ & $\begin{array}{c}{ }^{c} \text { Number average } \\
\text { diameter }(\mathrm{nm})\end{array}$ & 'PDI \\
\hline$x=0.05$ & 98 & 100 & 26400 & 1.39 & $119.2 \pm 9.1$ & $105.9 \pm 11.7$ & $100.2 \pm 17.2$ & $0.09 \pm 0.05$ \\
\hline$x=0.10$ & 98 & 100 & 25900 & 1.41 & $120.8 \pm 19.9$ & $90.2 \pm 13.4$ & $68.2 \pm 6.3$ & $0.12 \pm 0.05$ \\
\hline$x=0.15$ & 98 & 100 & 27800 & 1.30 & $102.8 \pm 23.8$ & $96.9 \pm 16.0$ & $83.8 \pm 5.1$ & $0.18 \pm 0.04$ \\
\hline$x=0.25$ & 93 & 100 & 28100 & 1.45 & $95.4 \pm 5.0$ & $79.3 \pm 6.3$ & $64.8 \pm 16.8$ & $0.09 \pm 0.02$ \\
\hline$x=0.40$ & 99 & 100 & 26400 & 1.90 & $258.5 \pm 32.8$ & $250.1 \pm 136.5$ & $87.6 \pm 33.5$ & $0.26 \pm 0.01$ \\
\hline$x=0.60$ & 97 & 100 & 28400 & 1.78 & $439.4 \pm 79.3$ & $532.9 \pm 128.5$ & $126.1 \pm 44.3$ & $0.28 \pm 0.03$ \\
\hline
\end{tabular}

${ }^{a} x$ is the feed molar ratio of $A C M A E /(C M A+A C M A E)$. ${ }^{b}$ The conversion of CMA and $A C M A E$ obtained via ${ }^{1} H$ NMR spectra. ${ }^{c T h e}$ intensity-/volume-/number-average diameter and PDI of the resultant vesicles obtained via DLS, all the data are averaged over three times of measurements. 


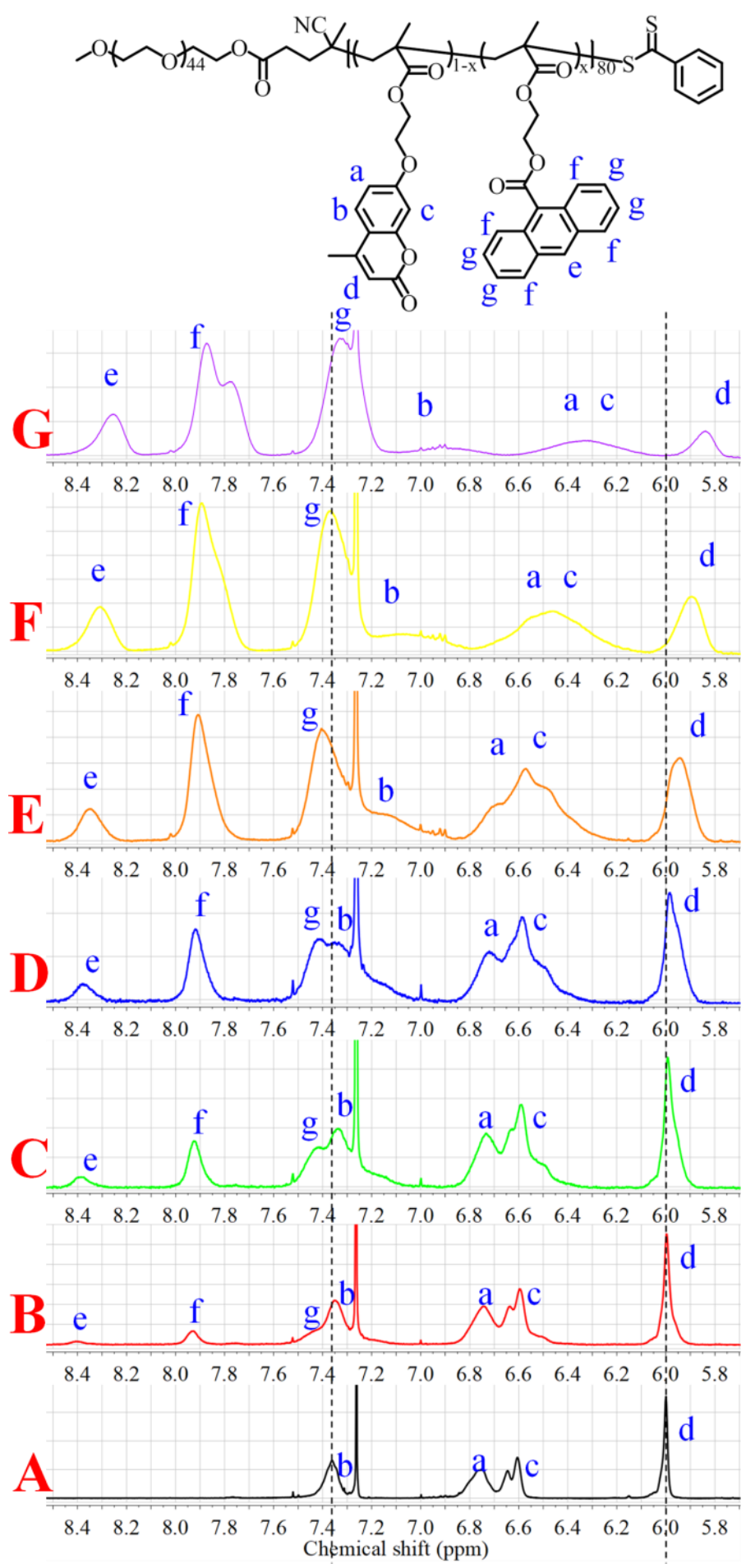

Fig. S19 ${ }^{1} \mathrm{H}$ NMR spectra of diblock terpolymers $P E O_{45}-b-\mathrm{P}\left(\mathrm{CMA}_{1-\mathrm{x}}-\mathrm{Co}^{-} \mathrm{ACMAEx}\right) 80$ obtained via RAFT dispersion copolymerization of CMA and ACMAE with different feed molar ratio of $A C M A E /(C M A+A C M A E),(A) x=0,(B) x=0.05,(C) x=0.10,(D) x=0.15$, $(E) x=0.25,(F) x=0.40$ and $(G) x=0.60$. The chemical shifts $(\delta)$ of $H_{b}$ and $H_{d}$ at $x=0$ were marked by the dash line, which shifted to lower $\delta$ values with increasing of the $x$ values. The chemical shifts $(\delta)$ of $H_{b}$ at $x \leq 0.15$ (the peaks of $H_{b}$ at $x>0.15$ are difficult to identified in these NMR spectra) and $\mathrm{H}_{\mathrm{d}}$ at various $\mathrm{x}$ values were summarized in Figure $5 \mathrm{C}$. 


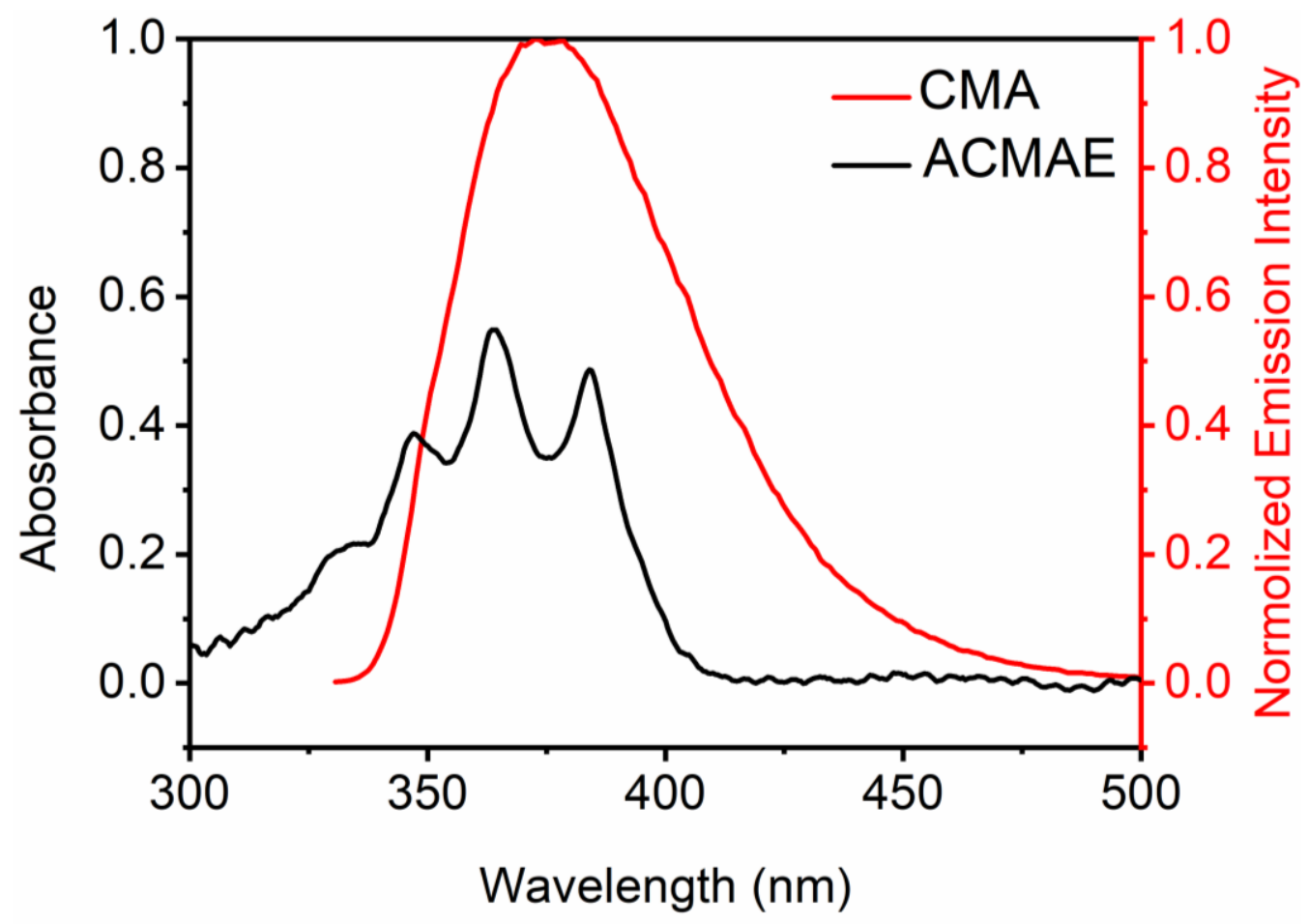

Fig. S20 Spectral overlap of emission spectrum of CMA (red line) and absorption spectrum of ACMAE (black line). 

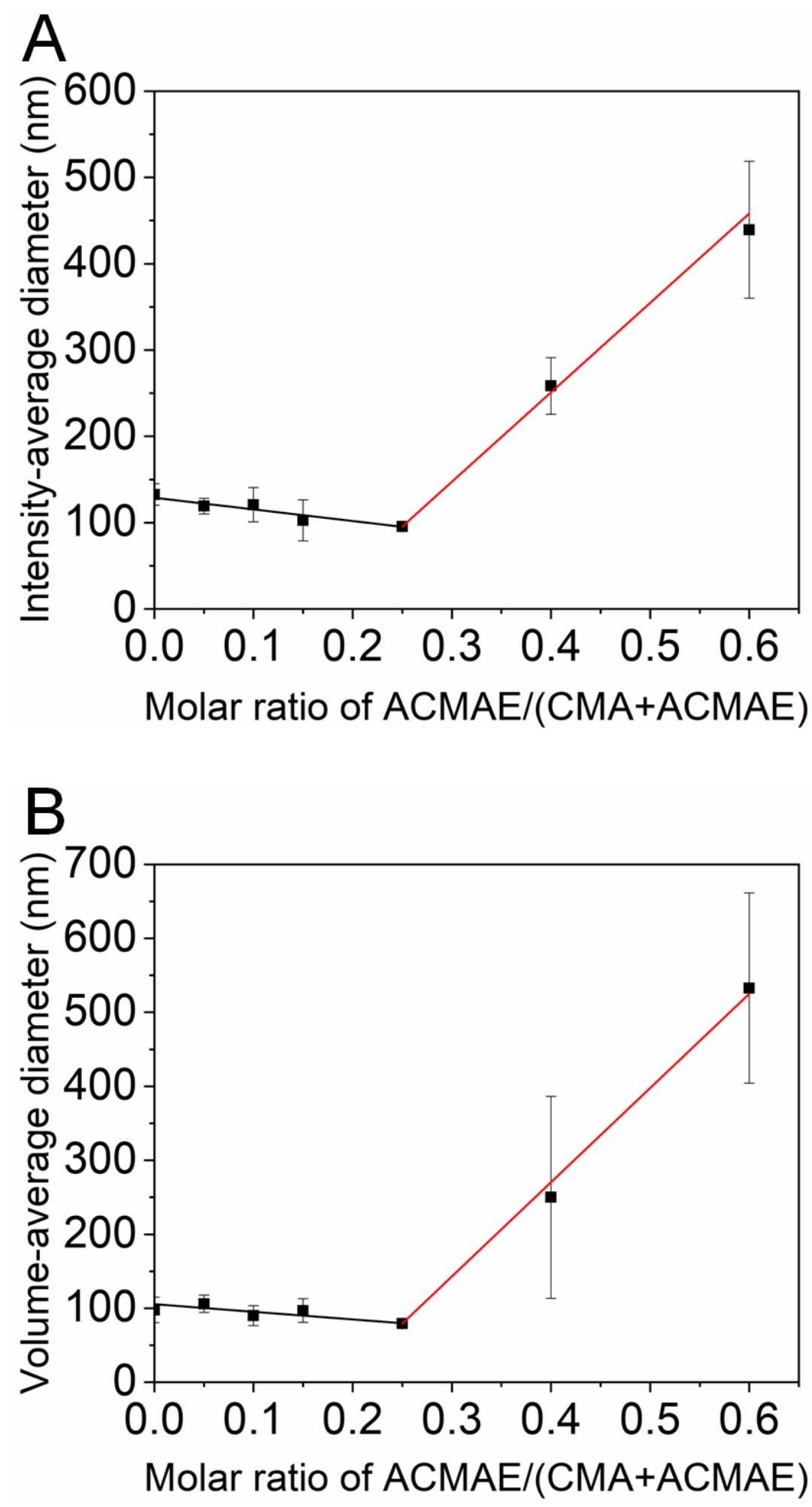

Fig. S21 (A) Intensity-average diameter and (B) volume-average diameter of

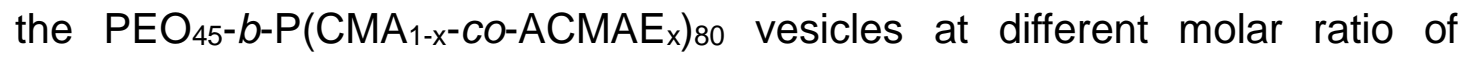
ACMAE/(CMA+ACMAE) ( $x$ values) obtained via DLS. 


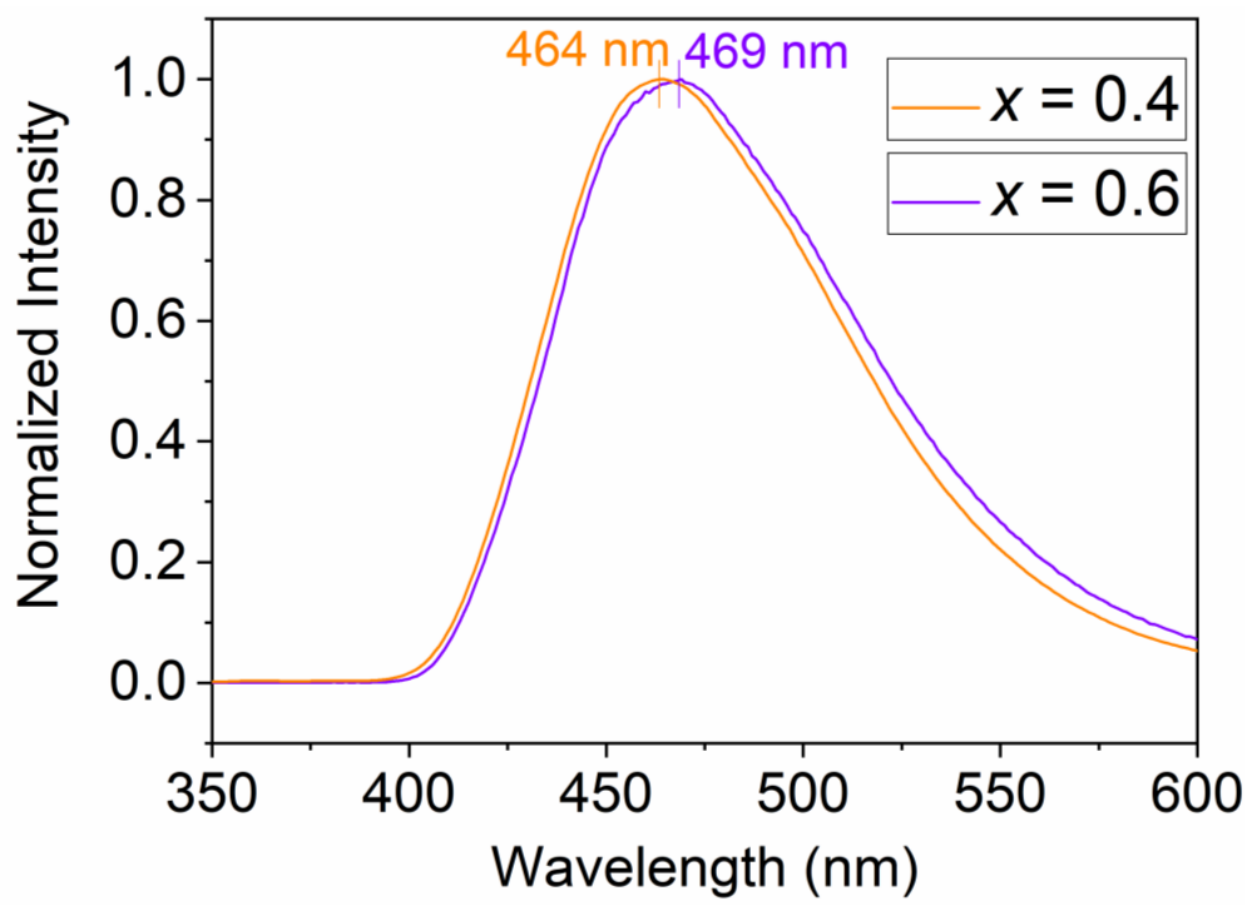

Fig. S22 Normalized fluorescence emission spectra of

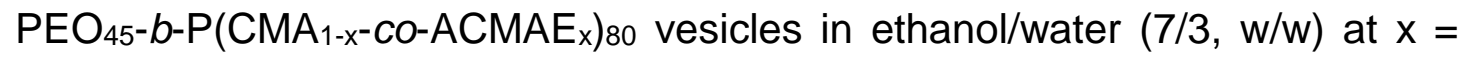
0.4 and $x=0.6$. 


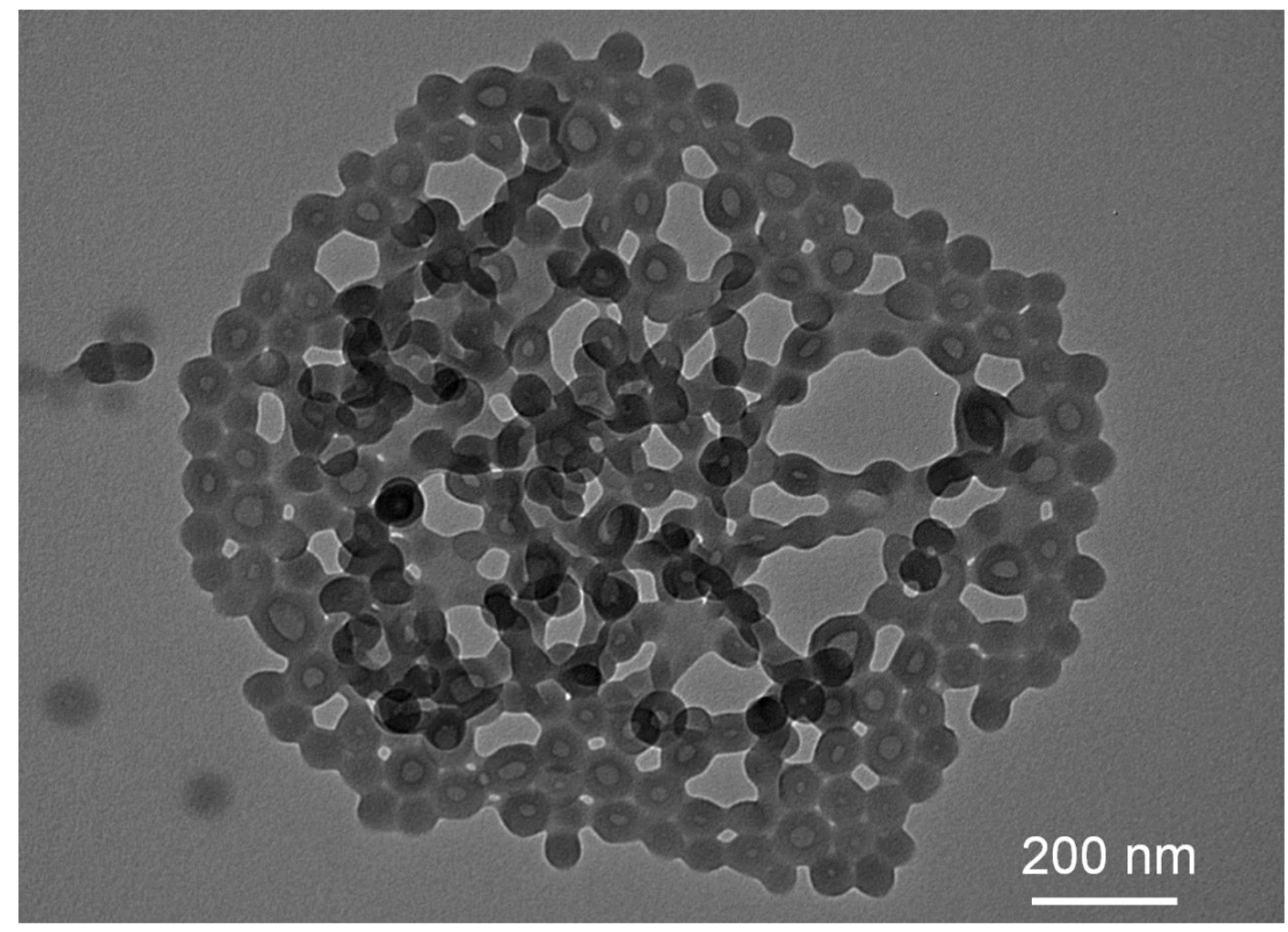

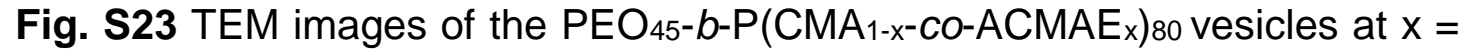
0.6 . 
Table S7. The details in feeds and monomer conversion for the fabrication of the PEO $45-b-P_{C M} A_{y}$ vesicles with varying DPPCMA (100, $120,150,200)$ at $5 \%$ or $10 \%$ solid content, or fixed chain concentration of $3.01 \times 10^{-3} \mathrm{mmol} / \mathrm{g}$.

\begin{tabular}{|c|c|c|c|c|c|c|}
\hline Samples & $\mathrm{PEO}_{45}-\mathrm{CPADB}$ & CMA & Ethanol/water $(7 / 3)$ & Solid content & ${ }^{\mathrm{a}}$ chain concentration & ${ }^{\mathrm{b}}$ Conversion of CMA (\%) \\
\hline $\mathrm{PEO}_{45}-b-\mathrm{PCMA}_{100}$ & $11 \mathrm{mg}\left(5 \times 10^{-3} \mathrm{mmol}\right)$ & $144 \mathrm{mg}(0.50 \mathrm{mmol})$ & $2950 \mathrm{mg}$ & $5 \%$ & $1.69 \times 10^{-3} \mathrm{mmol} / \mathrm{g}$ & 97 \\
\hline $\mathrm{PEO}_{45}-b-\mathrm{PCMA}_{120}$ & $11 \mathrm{mg}\left(5 \times 10^{-3} \mathrm{mmol}\right)$ & $173 \mathrm{mg}(0.60 \mathrm{mmol})$ & $3500 \mathrm{mg}$ & $5 \%$ & $1.43 \times 10^{-3} \mathrm{mmol} / \mathrm{g}$ & 95 \\
\hline $\mathrm{PEO}_{45}-b-\mathrm{PCMA}_{150}$ & $11 \mathrm{mg}\left(5 \times 10^{-3} \mathrm{mmol}\right)$ & $216 \mathrm{mg}(0.75 \mathrm{mmol})$ & $4310 \mathrm{mg}$ & $5 \%$ & $1.16 \times 10^{-3} \mathrm{mmol} / \mathrm{g}$ & 99 \\
\hline $\mathrm{PEO}_{45}-b-\mathrm{PCMA}_{200}$ & $11 \mathrm{mg}\left(5 \times 10^{-3} \mathrm{mmol}\right)$ & $288 \mathrm{mg}(1.00 \mathrm{mmol})$ & $5680 \mathrm{mg}$ & $5 \%$ & $0.88 \times 10^{-3} \mathrm{mmol} / \mathrm{g}$ & 98 \\
\hline $\mathrm{PEO}_{45}-b-\mathrm{PCMA}_{100}$ & $11 \mathrm{mg}\left(5 \times 10^{-3} \mathrm{mmol}\right)$ & $144 \mathrm{mg}(0.50 \mathrm{mmol})$ & $1400 \mathrm{mg}$ & $10 \%$ & $3.57 \times 10^{-3} \mathrm{mmol} / \mathrm{g}$ & 99 \\
\hline $\mathrm{PEO}_{45}-b-\mathrm{PCMA}_{120}$ & $11 \mathrm{mg}\left(5 \times 10^{-3} \mathrm{mmol}\right)$ & $173 \mathrm{mg}(0.60 \mathrm{mmol})$ & $1660 \mathrm{mg}$ & $10 \%$ & $3.01 \times 10^{-3} \mathrm{mmol} / \mathrm{g}$ & 99 \\
\hline $\mathrm{PEO}_{45}-b-\mathrm{PCMA}_{150}$ & $11 \mathrm{mg}\left(5 \times 10^{-3} \mathrm{mmol}\right)$ & $216 \mathrm{mg}(0.75 \mathrm{mmol})$ & $2040 \mathrm{mg}$ & $10 \%$ & $2.45 \times 10^{-3} \mathrm{mmol} / \mathrm{g}$ & 99 \\
\hline $\mathrm{PEO}_{45}-b-\mathrm{PCMA}_{200}$ & $11 \mathrm{mg}\left(5 \times 10^{-3} \mathrm{mmol}\right)$ & $288 \mathrm{mg}(1.00 \mathrm{mmol})$ & $2690 \mathrm{mg}$ & $10 \%$ & $1.86 \times 10^{-3} \mathrm{mmol} / \mathrm{g}$ & 97 \\
\hline $\mathrm{PEO}_{45}-b-\mathrm{PCMA}_{150}$ & $11 \mathrm{mg}\left(5 \times 10^{-3} \mathrm{mmol}\right)$ & $216 \mathrm{mg}(0.75 \mathrm{mmol})$ & $1660 \mathrm{mg}$ & - & $3.01 \times 10^{-3} \mathrm{mmol} / \mathrm{g}$ & 100 \\
\hline $\mathrm{PEO}_{45}-b-\mathrm{PCMA}_{200}$ & $11 \mathrm{mg}\left(5 \times 10^{-3} \mathrm{mmol}\right)$ & $288 \mathrm{mg}(1.00 \mathrm{mmol})$ & $1660 \mathrm{mg}$ & - & $3.01 \times 10^{-3} \mathrm{mmol} / \mathrm{g}$ & 100 \\
\hline
\end{tabular}

aThe concentration of the polymer chain is roughly considered to be the same as the concentration of the PEO $45-C P A D B .{ }^{b} T h e$ conversion of CMA obtained via ${ }^{1} \mathrm{H}$ NMR spectra. 

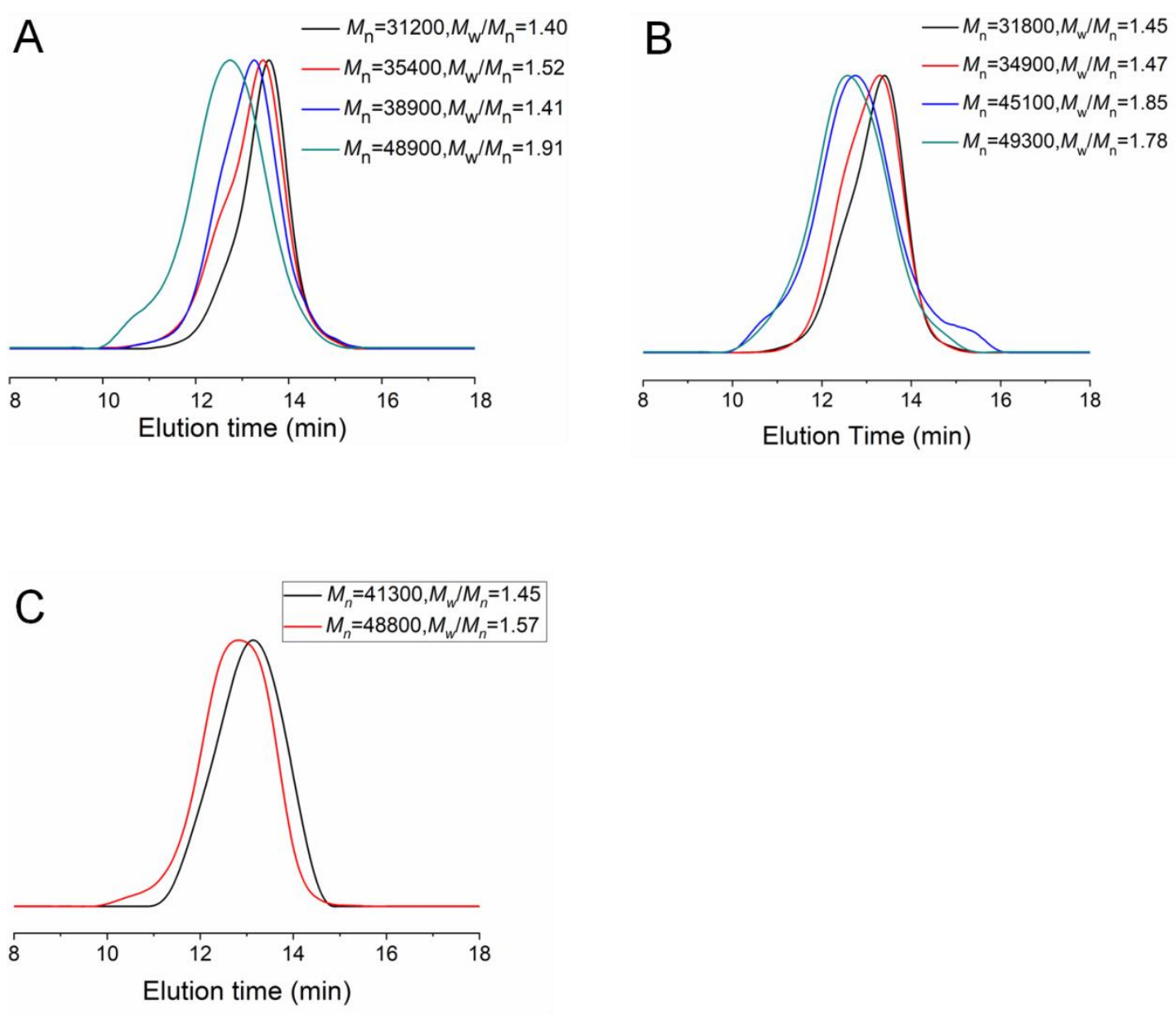

Fig. S24 GPC traces of the block copolymers $\mathrm{PEO}_{45}-b-\mathrm{PCMA}_{100} 200$ obtained at (A) 5\% solid content, (B) 10\% solid content, and (C) PEO $\mathrm{PE}_{45}-b-\mathrm{PCMA}_{150}$ and $\mathrm{PEO}_{45}-b-\mathrm{PCMA}_{200}$ obtained at a fixed chain concentration of $3.01 \times 10^{-3}$ $\mathrm{mmol} / \mathrm{g}$. 

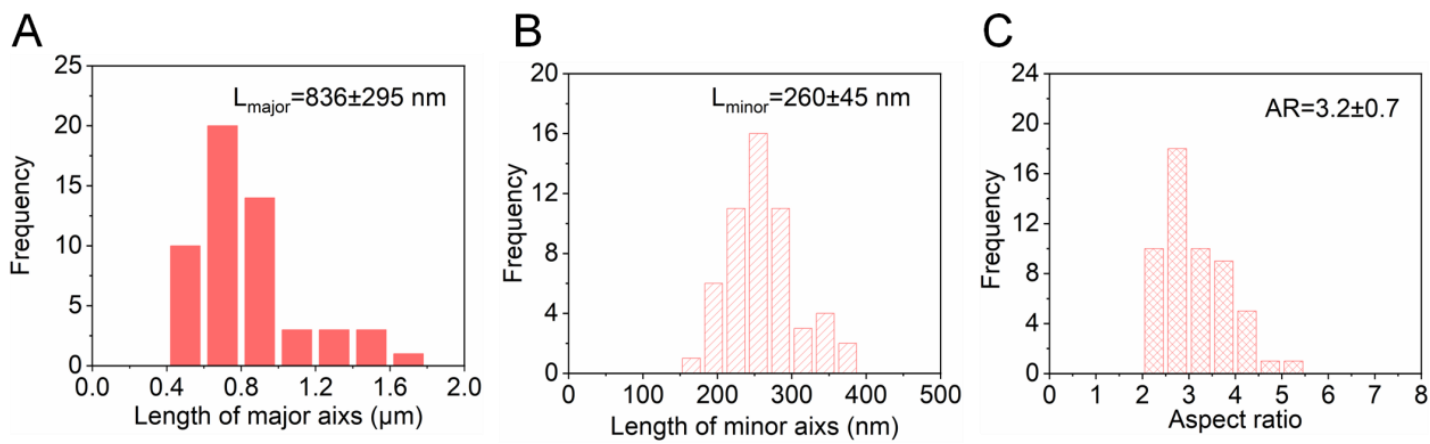

Fig. S25 Representative histograms for the (A) major axis lengths, $(B)$ minor axis lengths (width) and $(C)$ aspect ratios of the $\mathrm{PEO}_{45}-b-\mathrm{PCMA}_{120}$ nanotubes obtained at $10 \%$ solid content (chain concentration of $3.01 \times 10^{-3} \mathrm{mmol} / \mathrm{g}$ ). Statistical analyses $(n=50)$ of the populations of these nanotubes on the basis of TEM observations using an open source image processing software (ImageJ).
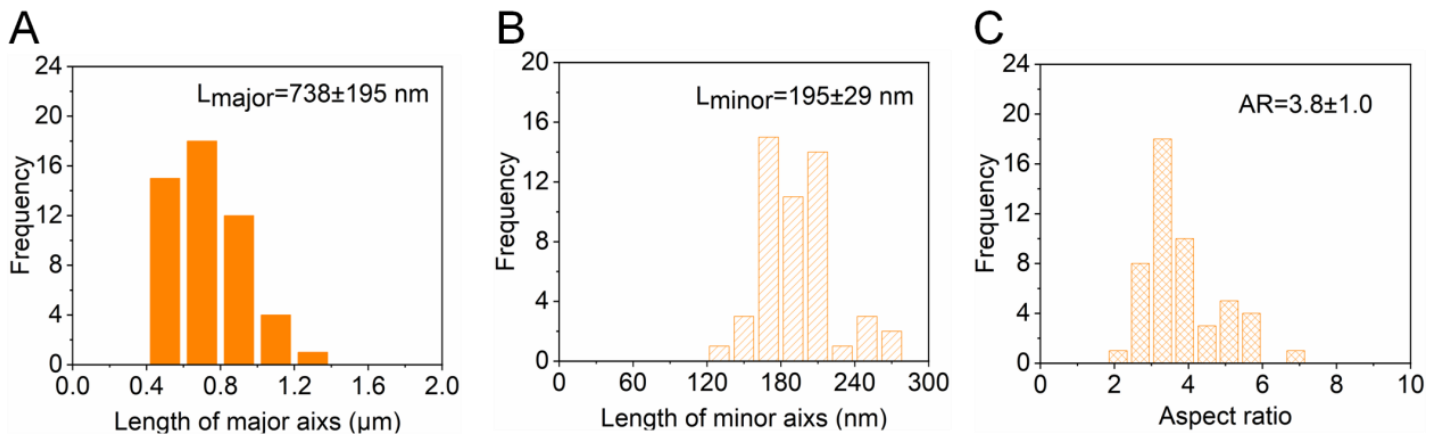

Fig. S26 Representative histograms for the (A) major axis lengths, (B) minor axis lengths (width) and $(C)$ aspect ratios of the $\mathrm{PEO}_{45}-b-\mathrm{PCMA}_{150}$ nanotubes obtained at $10 \%$ solid content (chain concentration of $2.45 \times 10^{-3} \mathrm{mmol} / \mathrm{g}$ ). Statistical analyses $(n=50)$ of the populations of these nanotubes on the basis of TEM observations using an open source image processing software (Image J). 

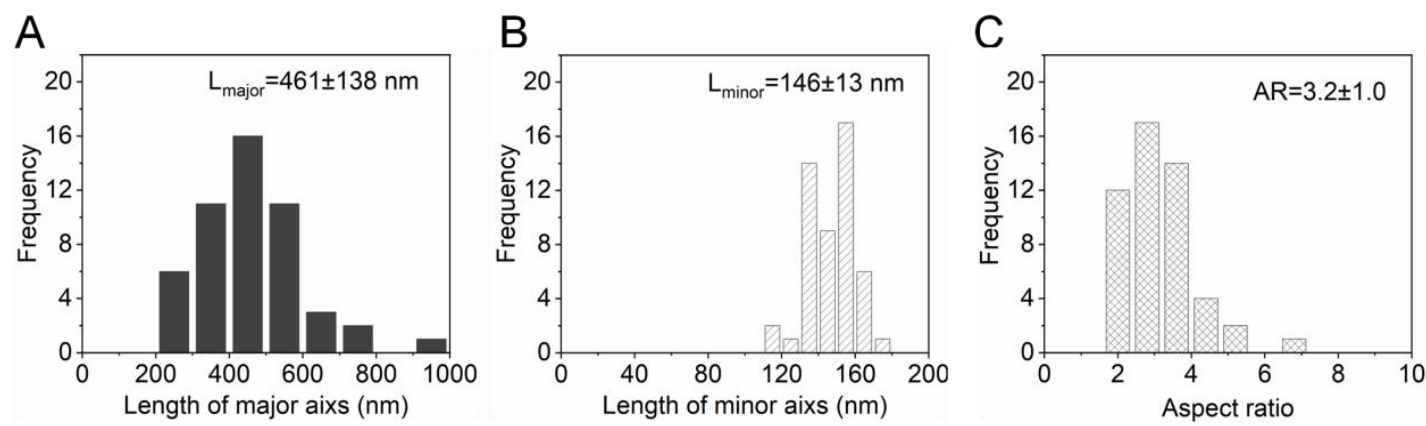

Fig. S27 Representative histograms for the (A) major axis lengths, (B) minor axis lengths (width) and (C) aspect ratios of the $\mathrm{PEO}_{45}-b-\mathrm{PCMA}_{200}$ nanotubes obtained at $10 \%$ solid content (chain concentration of $1.86 \times 10^{-3} \mathrm{mmol} / \mathrm{g}$ ). Statistical analyses $(n=50)$ of the populations of these nanotubes on the basis of TEM observations using an open source image processing software (ImageJ).
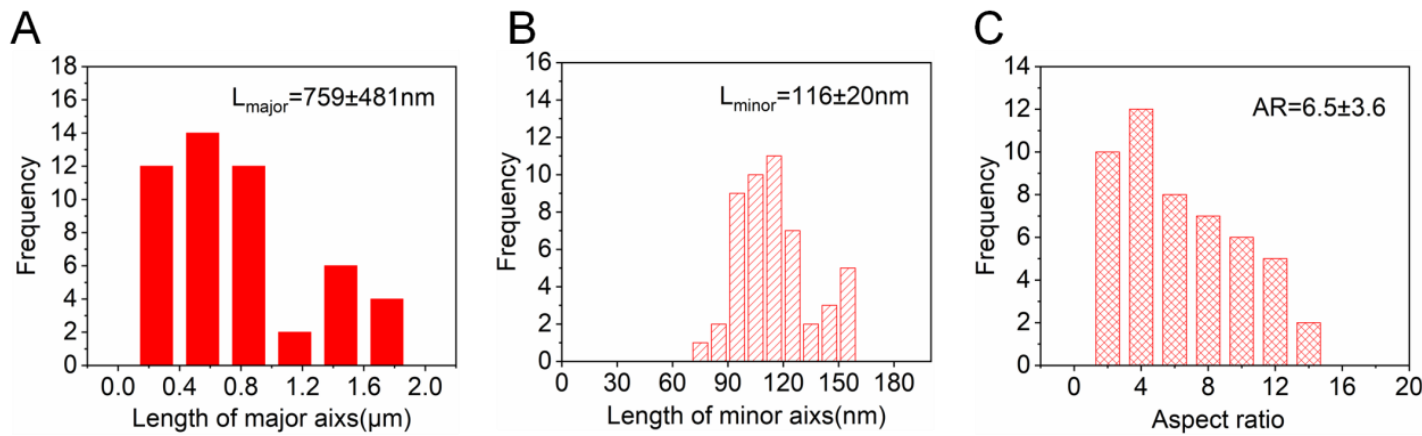

Fig. S28 Representative histograms for the (A) major axis lengths, (B) minor axis lengths (width) and (C) aspect ratios of the $\mathrm{PEO}_{45}-b-\mathrm{PCMA}_{150}$ nanotubes obtained at the chain concentration of $3.01 \times 10^{-3} \mathrm{mmol} / \mathrm{g}$. Statistical analyses $(n=50)$ of the populations of these nanotubes on the basis of TEM observations using an open source image processing software (Image J). 

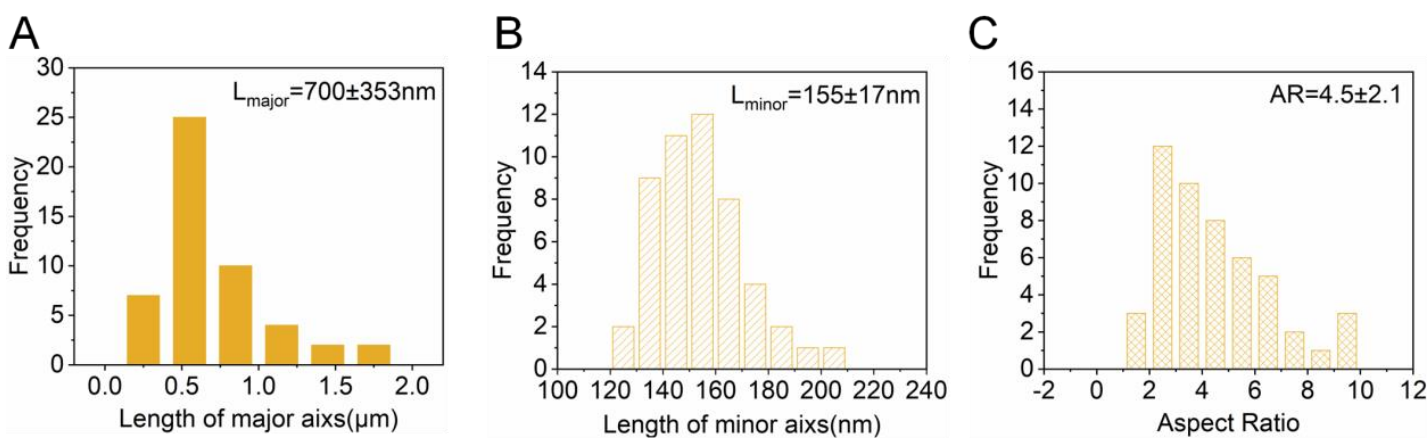

Fig. S29 Representative histograms for the (A) major axis lengths, (B) minor axis lengths (width) and (C) aspect ratios of the $\mathrm{PEO}_{45}-b-\mathrm{PCMA}_{200}$ nanotubes obtained at the chain concentration of $3.01 \times 10^{-3} \mathrm{mmol} / \mathrm{g}$. Statistical analyses $(n=50)$ of the populations of these nanotubes on the basis of TEM observations using an open source image processing software (ImageJ). 


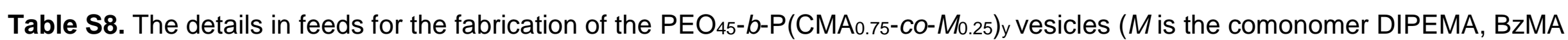
or ACMAE) at a fixed chain concentration of $3.01 \times 10^{-3} \mathrm{mmol} / \mathrm{g}$ with varying DP of the solvophobic block $(80,100,120,150,200)$.

\begin{tabular}{|c|c|c|c|c|c|c|c|}
\hline Samples & PEO $_{45}$-CPADB & CMA & DIPEMA/BzMA/ACMAE & $\begin{array}{c}\text { Ethanol/water } \\
(7 / 3)\end{array}$ & aChain concentration & $\begin{array}{c}\mathrm{b} \text { Conversion of } \\
\text { CMA (\%) }\end{array}$ & $\begin{array}{c}\text { bConversion of } \\
\text { commoner } M(\%)\end{array}$ \\
\hline $\mathrm{PEO}_{45}-b-\mathrm{P}\left(\mathrm{CMA}_{0.75} \text {-co-DIPEMA } \mathrm{A}_{0.25}\right)_{80}$ & $11 \mathrm{mg}\left(5 \times 10^{-3} \mathrm{mmol}\right)$ & $86 \mathrm{mg}(0.300 \mathrm{mmol})$ & DIPEMA (21 mg, 0.100mmol) & $1660 \mathrm{mg}$ & $3.01 \times 10^{-3} \mathrm{mmol} / \mathrm{g}$ & 95 & 91 \\
\hline $\mathrm{PEO}_{45}-b$-P(CMA $A_{0.75}$-co-DIPEMA $\left.{ }_{0.25}\right)_{100}$ & $11 \mathrm{mg}\left(5 \times 10^{-3} \mathrm{mmol}\right)$ & $108 \mathrm{mg}(0.375 \mathrm{mmol})$ & DIPEMA (27 mg, 0.125mmol) & $1660 \mathrm{mg}$ & $3.01 \times 10^{-3} \mathrm{mmol} / \mathrm{g}$ & 94 & 91 \\
\hline $\mathrm{PEO}_{45}-b-\mathrm{P}\left(\mathrm{CM}_{0.75} \text {-co-DIPEMA } 0_{0.25}\right)_{120}$ & $11 \mathrm{mg}\left(5 \times 10^{-3} \mathrm{mmol}\right)$ & $130 \mathrm{mg}(0.450 \mathrm{mmol})$ & DIPEMA (32 mg, 0.150mmol) & $1660 \mathrm{mg}$ & $3.01 \times 10^{-3} \mathrm{mmol} / \mathrm{g}$ & 93 & 85 \\
\hline $\mathrm{PEO}_{45}-b-\mathrm{P}\left(\mathrm{CMA}_{0.75} \text {-co-DIPEMA }{ }_{0.25}\right)_{150}$ & $11 \mathrm{mg}\left(5 \times 10^{-3} \mathrm{mmol}\right)$ & $162 \mathrm{mg}(0.563 \mathrm{mmol})$ & DIPEMA (40 mg, 0.187mmol) & $1660 \mathrm{mg}$ & $3.01 \times 10^{-3} \mathrm{mmol} / \mathrm{g}$ & 96 & 88 \\
\hline $\mathrm{PEO}_{45}-b-\mathrm{P}\left(\mathrm{CM}_{0.75} \text {-co-DIPEMA } \mathrm{A}_{0.25}\right)_{200}$ & $11 \mathrm{mg}\left(5 \times 10^{-3} \mathrm{mmol}\right)$ & $216 \mathrm{mg}(0.750 \mathrm{mmol})$ & DIPEMA (53 mg, 0.250mmol) & $1660 \mathrm{mg}$ & $3.01 \times 10^{-3} \mathrm{mmol} / \mathrm{g}$ & 96 & 88 \\
\hline $\mathrm{PEO}_{45}-b-\mathrm{P}\left(\mathrm{CMA}_{0.75}-\mathrm{co}-\mathrm{BzMA}_{0.25}\right)_{80}$ & $11 \mathrm{mg}\left(5 \times 10^{-3} \mathrm{mmol}\right)$ & $86 \mathrm{mg}(0.300 \mathrm{mmol})$ & BzMA (18 mg, $0.100 \mathrm{mmol})$ & $1660 \mathrm{mg}$ & $3.01 \times 10^{-3} \mathrm{mmol} / \mathrm{g}$ & 97 & 96 \\
\hline $\mathrm{PEO}_{45}-b-\mathrm{P}\left(\mathrm{CM} \mathrm{A}_{0.75}-\mathrm{co}-\mathrm{Bz}_{\mathrm{MA}} \mathrm{A}_{0.25}\right)_{100}$ & $11 \mathrm{mg}\left(5 \times 10^{-3} \mathrm{mmol}\right)$ & $108 \mathrm{mg}(0.375 \mathrm{mmol})$ & BzMA (22 mg, 0.125mmol) & $1660 \mathrm{mg}$ & $3.01 \times 10^{-3} \mathrm{mmol} / \mathrm{g}$ & 98 & 99 \\
\hline $\mathrm{PEO}_{45}-b-\mathrm{P}\left(\mathrm{CMA}_{0.75}-\mathrm{co}-\mathrm{BzMA}_{0.25}\right)_{120}$ & $11 \mathrm{mg}\left(5 \times 10^{-3} \mathrm{mmol}\right)$ & $130 \mathrm{mg}(0.450 \mathrm{mmol})$ & BzMA (26 mg, $0.150 \mathrm{mmol})$ & $1660 \mathrm{mg}$ & $3.01 \times 10^{-3} \mathrm{mmol} / \mathrm{g}$ & 97 & 91 \\
\hline $\mathrm{PEO}_{45}-b-\mathrm{P}\left(\mathrm{CMA}_{0.75}-\mathrm{Co}-\mathrm{Bz}_{\mathrm{MA}} \mathrm{A}_{0.25}\right)_{150}$ & $11 \mathrm{mg}\left(5 \times 10^{-3} \mathrm{mmol}\right)$ & $162 \mathrm{mg}(0.563 \mathrm{mmol})$ & BzMA (33 mg, 0.187mmol) & $1660 \mathrm{mg}$ & $3.01 \times 10^{-3} \mathrm{mmol} / \mathrm{g}$ & 98 & 94 \\
\hline $\mathrm{PEO}_{45}-b-\mathrm{P}\left(\mathrm{CMA}_{0.75}-\mathrm{Co}-\mathrm{Bz} \mathrm{MA}_{0.25}\right)_{200}$ & $11 \mathrm{mg}\left(5 \times 10^{-3} \mathrm{mmol}\right)$ & $216 \mathrm{mg}(0.750 \mathrm{mmol})$ & BzMA (44mg, $0.250 \mathrm{mmol})$ & $1660 \mathrm{mg}$ & $3.01 \times 10^{-3} \mathrm{mmol} / \mathrm{g}$ & 95 & 97 \\
\hline $\mathrm{PEO}_{45}-b-\mathrm{P}\left(\mathrm{CMA}_{0.75}-\mathrm{Co}-\mathrm{ACMAE}_{0.25}\right)_{80}$ & $11 \mathrm{mg}\left(5 \times 10^{-3} \mathrm{mmol}\right)$ & $86 \mathrm{mg}(0.300 \mathrm{mmol})$ & ACMAE (33 mg, 0.100mmol) & $1660 \mathrm{mg}$ & $3.01 \times 10^{-3} \mathrm{mmol} / \mathrm{g}$ & 93 & 100 \\
\hline $\mathrm{PEO}_{45}-b-\mathrm{P}\left(\mathrm{CMA}_{0.75} \text {-co-ACMAE } \mathrm{E}_{0.25}\right)_{100}$ & $11 \mathrm{mg}\left(5 \times 10^{-3} \mathrm{mmol}\right)$ & $108 \mathrm{mg}(0.375 \mathrm{mmol})$ & ACMAE (42mg, $0.125 \mathrm{mmol})$ & $1660 \mathrm{mg}$ & $3.01 \times 10^{-3} \mathrm{mmol} / \mathrm{g}$ & 95 & 100 \\
\hline $\mathrm{PEO}_{45}-b-\mathrm{P}\left(\mathrm{CMA}_{0.75}-\mathrm{CO}-\mathrm{ACMAE}_{0.25}\right)_{120}$ & $11 \mathrm{mg}\left(5 \times 10^{-3} \mathrm{mmol}\right)$ & $130 \mathrm{mg}(0.450 \mathrm{mmol})$ & ACMAE (50 mg, $0.150 \mathrm{mmol})$ & $1660 \mathrm{mg}$ & $3.01 \times 10^{-3} \mathrm{mmol} / \mathrm{g}$ & 100 & 100 \\
\hline $\mathrm{PEO}_{45}-b-\mathrm{P}\left(\mathrm{CM}_{0.75}-\mathrm{Co}-\mathrm{ACMAE}_{0.25}\right)_{150}$ & $11 \mathrm{mg}\left(5 \times 10^{-3} \mathrm{mmol}\right)$ & $162 \mathrm{mg}(0.563 \mathrm{mmol})$ & ACMAE (62mg, $0.187 \mathrm{mmol})$ & $1660 \mathrm{mg}$ & $3.01 \times 10^{-3} \mathrm{mmol} / \mathrm{g}$ & 100 & 100 \\
\hline $\mathrm{PEO}_{45}-b-\mathrm{P}\left(\mathrm{CMA}_{0.75}-\mathrm{Co}-\mathrm{ACMAE}_{0.25}\right)_{200}$ & $11 \mathrm{mg}\left(5 \times 10^{-3} \mathrm{mmol}\right)$ & $216 \mathrm{mg}(0.750 \mathrm{mmol})$ & ACMAE (84mg, $0.250 \mathrm{mmol})$ & $1660 \mathrm{mg}$ & $3.01 \times 10^{-3} \mathrm{mmol} / \mathrm{g}$ & 100 & 100 \\
\hline
\end{tabular}

aThe concentration of the polymer chain is roughly considered to be the same as the concentration of the PEO $45-\mathrm{CPADB}^{\mathrm{b}} \mathrm{b}^{\mathrm{T}} \mathrm{C}$ conversion of CMA and comonomer (DIPEMA, BzMA or ACMAE) obtained via ${ }^{1} \mathrm{H}$ NMR spectra. 

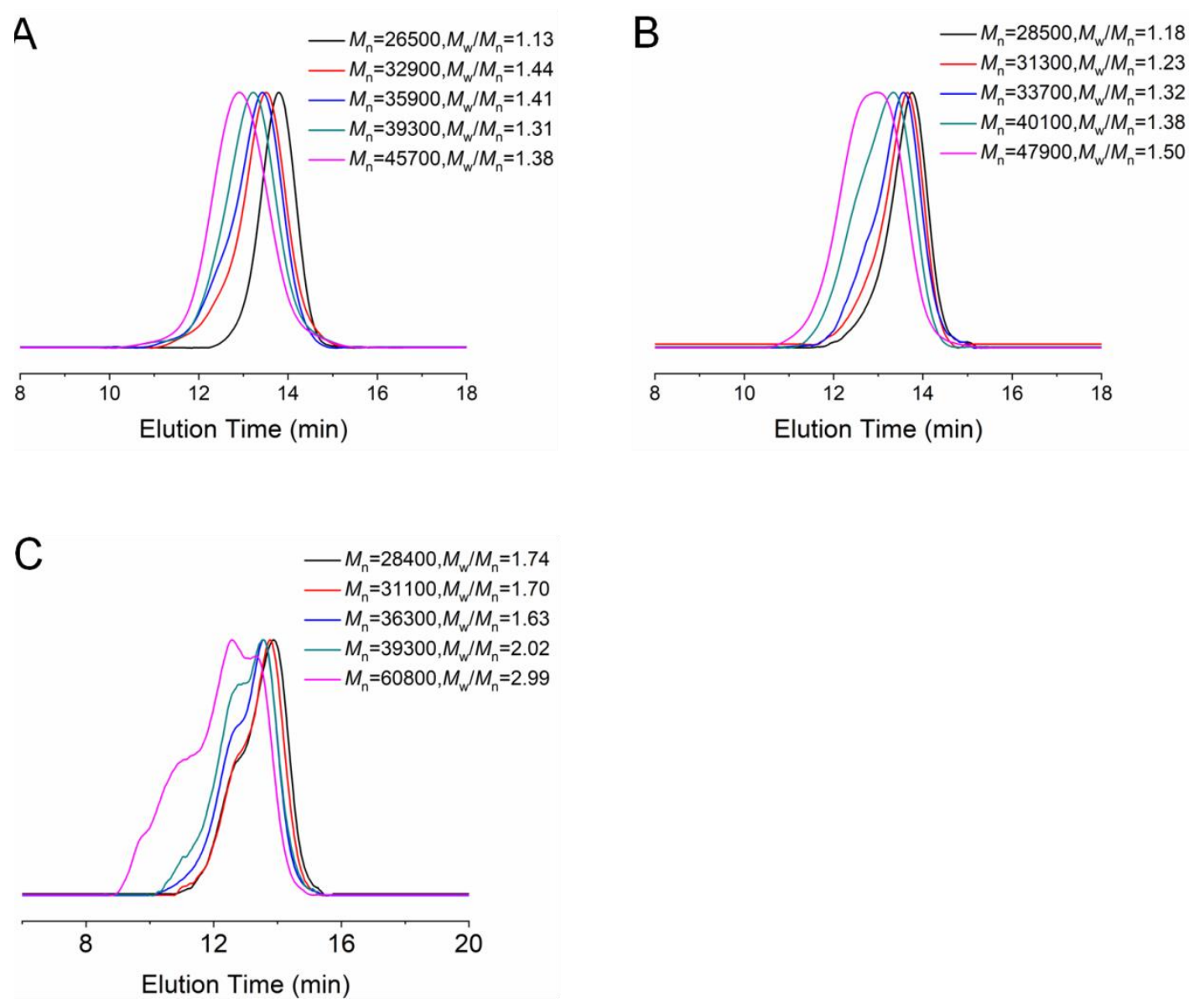

Fig. S30 GPC traces of the block copolymers (A)

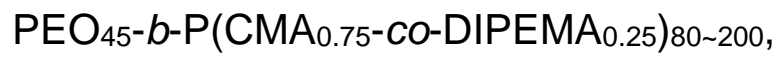

(B) $\quad \mathrm{PEO}_{45}-b-\mathrm{P}\left(\mathrm{CMA}_{0.75-c o-}\right.$

BzMA $\left._{0.25)}\right)_{80 \sim 200,}$ (C) PEO $45-b-\mathrm{P}\left(\mathrm{CMA}_{0.75}-\mathrm{Co}-\mathrm{ACMAE}_{0.25}\right)_{80 \sim 200 .}$ 

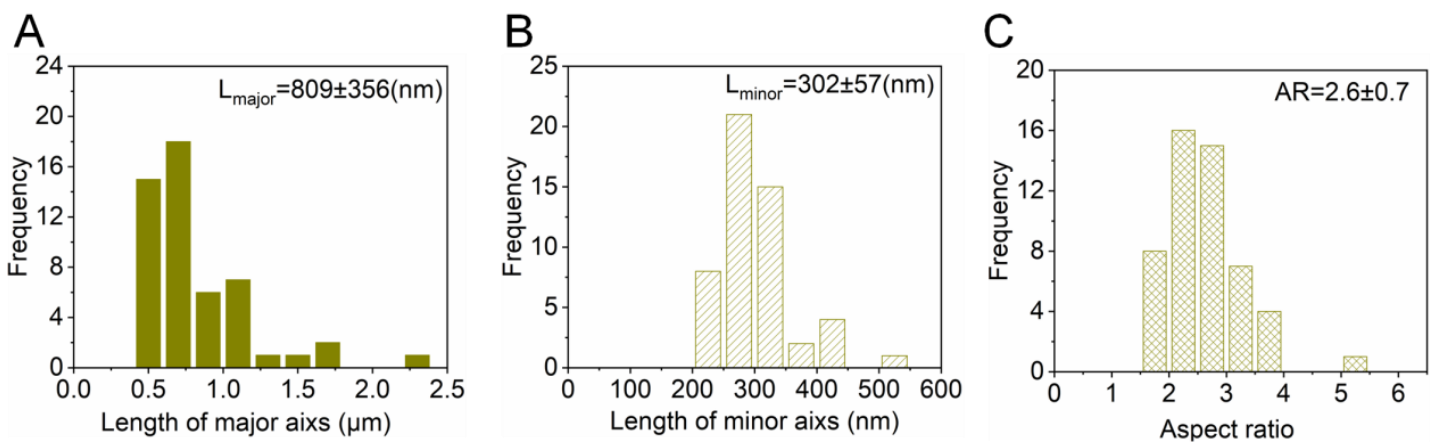

Fig. S31 Representative histograms for the (A) major axis lengths, $(B)$ minor axis lengths (width) and $(\mathrm{C})$ aspect ratios of the

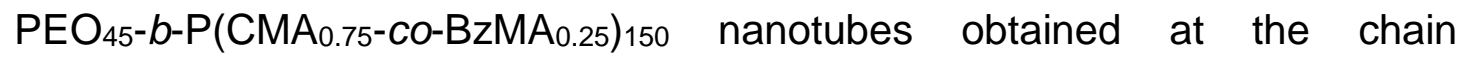
concentration of $3.01 \times 10^{-3} \mathrm{mmol} / \mathrm{g}$. Statistical analyses $(\mathrm{n}=50)$ of the populations of these nanotubes on the basis of TEM observations using an open source image processing software (ImageJ).

A

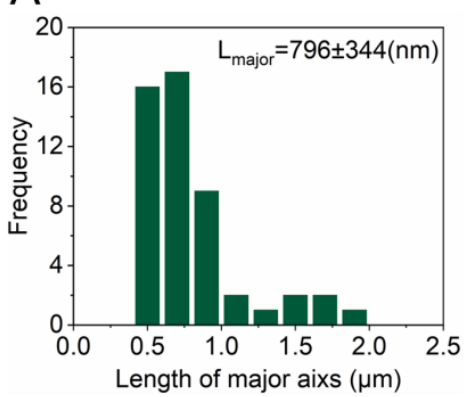

$\mathrm{B}$

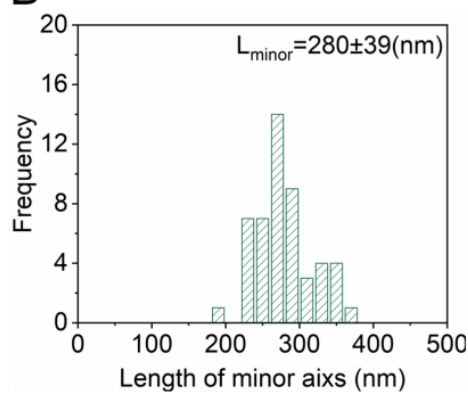

C

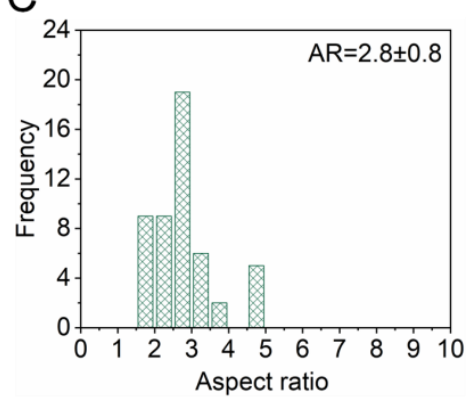

Fig. S32 Representative histograms for the (A) major axis lengths, (B) minor axis lengths (width) and (C) aspect ratios of the

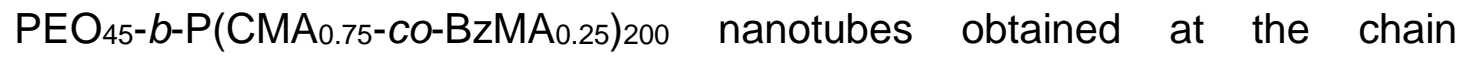
concentration of $3.01 \times 10^{-3} \mathrm{mmol} / \mathrm{g}$. Statistical analyses $(n=50)$ of the populations of these nanotubes on the basis of TEM observations using an open source image processing software (ImageJ). 
A

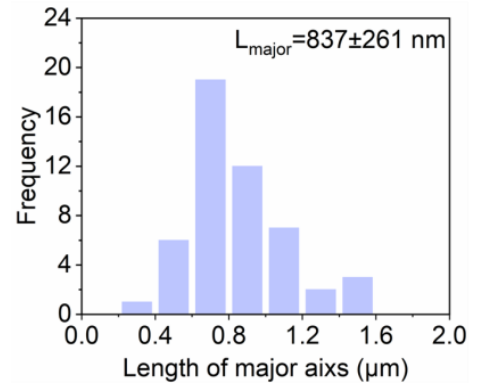

B

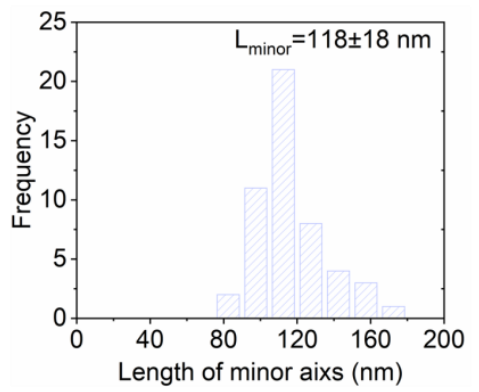

C

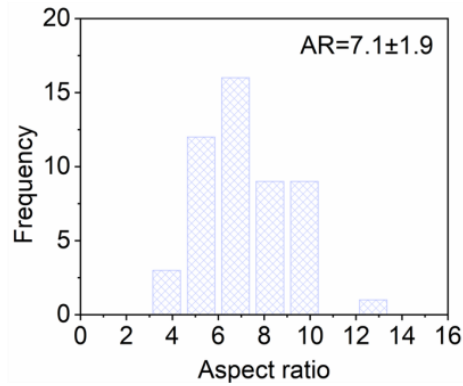

Fig. S33 Representative histograms for the (A) major axis lengths, (B) minor axis lengths (width) and (C) aspect ratios of the

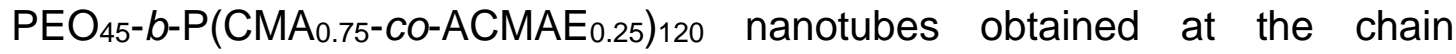
concentration of $3.01 \times 10^{-3} \mathrm{mmol} / \mathrm{g}$. Statistical analyses $(\mathrm{n}=50)$ of the populations of these nanotubes on the basis of TEM observations using an open source image processing software (ImageJ).

A

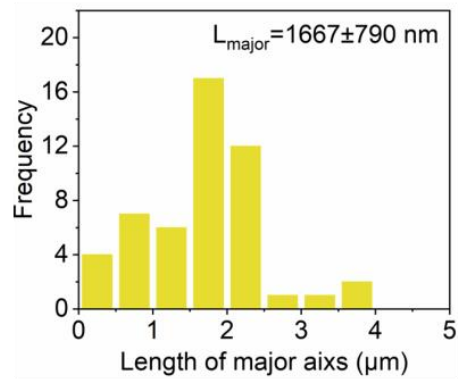

B

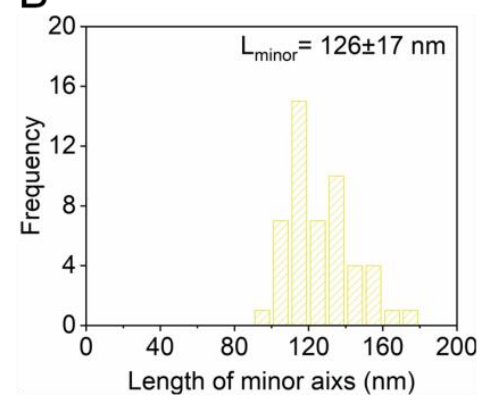

C

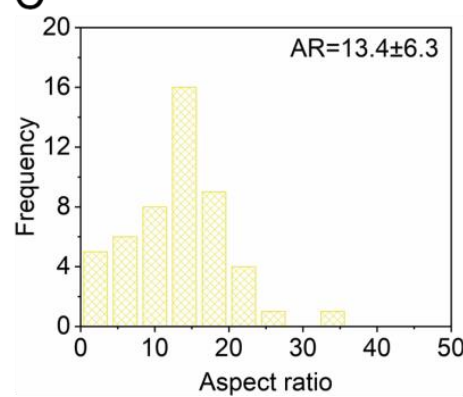

Fig. S34 Representative histograms for the (A) major axis lengths, (B) minor axis lengths (width) and (C) aspect ratios of the

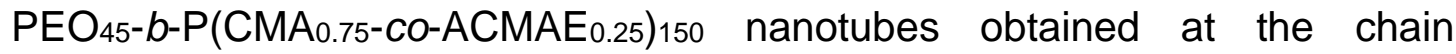
concentration of $3.01 \times 10^{-3} \mathrm{mmol} / \mathrm{g}$. Statistical analyses $(\mathrm{n}=50)$ of the populations of these nanotubes on the basis of TEM observations using an open source image processing software (ImageJ). 

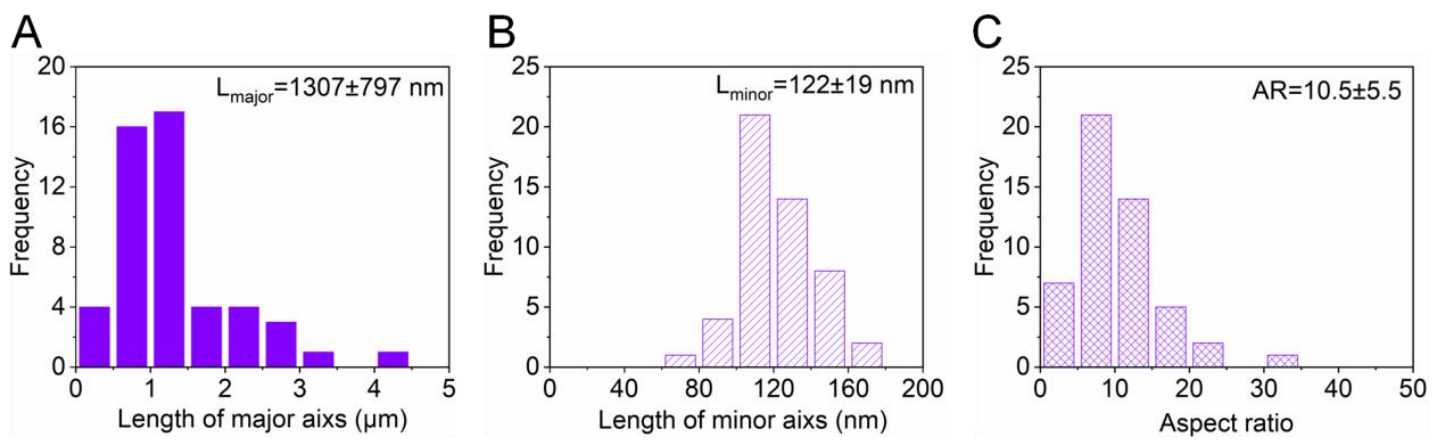

Fig. S35 Representative histograms for the (A) major axis lengths, (B) minor axis lengths (width) and (C) aspect ratios of the

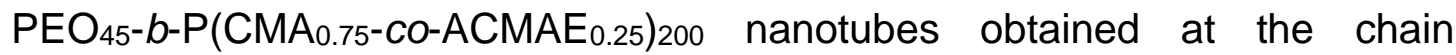
concentration of $3.01 \times 10^{-3} \mathrm{mmol} / \mathrm{g}$. Statistical analyses $(n=50)$ of the populations of these nanotubes on the basis of TEM observations using an open source image processing software (ImageJ). 
Table S9. The details in feeds for the fabrication of the $\mathrm{PEO}_{45}-\mathrm{b}-\mathrm{P}\left(\mathrm{CMA} \mathrm{A}_{0.6}-\mathrm{Co}-\mathrm{M}_{0.4}\right)$ y vesicles ( $M$ is the comonomer DIPEMA, BzMA or ACMAE) at a fixed chain concentration of $3.01 \times 10^{-3} \mathrm{mmol} / \mathrm{g}$ with varying DP of the solvophobic block $(80,100,120,150,200)$.

\begin{tabular}{|c|c|c|c|c|c|c|c|}
\hline Samples & $\mathrm{PEO}_{45}$-CPADB & CMA & DIPEMA/BzMA/ACMAE & $\begin{array}{c}\text { Ethanol/water } \\
(7 / 3)\end{array}$ & ${ }^{a}$ Chain concentration & $\begin{array}{c}\begin{array}{c}\mathrm{b} C o n v e r s i o n \\
\text { CMA (\%) }\end{array} \\
\end{array}$ & $\begin{array}{c}{ }^{\mathrm{b}} \text { Conversion of } \\
\text { comonmer } \boldsymbol{M}(\%)\end{array}$ \\
\hline $\mathrm{PEO}_{45}-b-\mathrm{P}\left(\mathrm{CM} \mathrm{A}_{0.6-6} \text {-Co-DIPEMA } \mathrm{A}_{0.4}\right)_{80}$ & $11 \mathrm{mg}\left(5 \times 10^{-3} \mathrm{mmol}\right)$ & $69 \mathrm{mg}, 0.24 \mathrm{mmol}$ & DIPEMA (34 mg, $0.16 \mathrm{mmol}$ ) & $1660 \mathrm{mg}$ & $3.01 \times 10^{-3} \mathrm{mmol} / \mathrm{g}$ & 87 & 84 \\
\hline 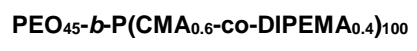 & $11 \mathrm{mg}\left(5 \times 10^{-3} \mathrm{mmol}\right)$ & $86 \mathrm{mg}, 0.30 \mathrm{mmol}$ & DIPEMA (43 mg, $0.20 \mathrm{mmol}$ ) & $1660 \mathrm{mg}$ & $3.01 \times 10^{-3} \mathrm{mmol} / \mathrm{g}$ & 83 & 84 \\
\hline $\mathrm{PEO}_{45-b-\mathrm{P}}\left(\mathrm{CMA}_{0.6}-\mathrm{-} \text {-Co-DIPEMA } \mathrm{A}_{0.4}\right)_{120}$ & $11 \mathrm{mg}\left(5 \times 10^{-3} \mathrm{mmol}\right)$ & $104 \mathrm{mg}, 0.36 \mathrm{mmol}$ & DIPEMA (51 mg, $0.24 \mathrm{mmol}$ ) & $1660 \mathrm{mg}$ & $3.01 \times 10^{-3} \mathrm{mmol} / \mathrm{g}$ & 89 & 85 \\
\hline 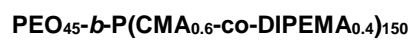 & $11 \mathrm{mg}\left(5 \times 10^{-3} \mathrm{mmol}\right)$ & $130 \mathrm{mg}, 0.45 \mathrm{mmol}$ & DIPEMA (64 mg, $0.30 \mathrm{mmol}$ ) & $1660 \mathrm{mg}$ & $3.01 \times 10^{-3} \mathrm{mmol} / \mathrm{g}$ & 97 & 83 \\
\hline $\mathrm{PEO}_{45}-b-\mathrm{P}\left(\mathrm{CMA}_{0.6} \text {-Co-DIPEMA }_{0.4}\right)_{200}$ & $11 \mathrm{mg}\left(5 \times 10^{-3} \mathrm{mmol}\right)$ & $173 \mathrm{mg}, 0.60 \mathrm{mmol}$ & DIPEMA ( $85 \mathrm{mg}, 0.40 \mathrm{mmol}$ ) & $1660 \mathrm{mg}$ & $3.01 \times 10^{-3} \mathrm{mmol} / \mathrm{g}$ & 96 & 81 \\
\hline $\mathrm{PEO}_{45}-b-\mathrm{P}\left(\mathrm{CMA}_{0.6}-\mathrm{Co}-\mathrm{BzMA}_{0.4}\right)_{80}$ & $11 \mathrm{mg}\left(5 \times 10^{-3} \mathrm{mmol}\right)$ & $69 \mathrm{mg}, 0.24 \mathrm{mmol}$ & $\mathrm{BzMA}(28 \mathrm{mg}, 0.16 \mathrm{mmol})$ & $1660 \mathrm{mg}$ & $3.01 \times 10^{-3} \mathrm{mmol} / \mathrm{g}$ & 99 & 97 \\
\hline $\mathrm{PEO}_{45}-b-\mathrm{P}\left(\mathrm{CMA}_{0.6}-\mathrm{-co}-\mathrm{Bz} \mathrm{MA}_{0.4}\right)_{100}$ & $11 \mathrm{mg}\left(5 \times 10^{-3} \mathrm{mmol}\right)$ & $86 \mathrm{mg}, 0.30 \mathrm{mmol}$ & $\mathrm{BzMA}(35 \mathrm{mg}, 0.20 \mathrm{mmol})$ & $1660 \mathrm{mg}$ & $3.01 \times 10^{-3} \mathrm{mmol} / \mathrm{g}$ & 98 & 99 \\
\hline $\mathrm{PEO}_{45}-b-\mathrm{P}\left(\mathrm{CMA}_{0.6}-\mathrm{co}-\mathrm{Bz} \mathrm{MA}_{0.4}\right)_{120}$ & $11 \mathrm{mg}\left(5 \times 10^{-3} \mathrm{mmol}\right)$ & $104 \mathrm{mg}, 0.36 \mathrm{mmol}$ & $\mathrm{BzMA}(42 \mathrm{mg}, 0.24 \mathrm{mmol})$ & $1660 \mathrm{mg}$ & $3.01 \times 10^{-3} \mathrm{mmol} / \mathrm{g}$ & 98 & 99 \\
\hline $\mathrm{PEO}_{45}-b-\mathrm{P}\left(\mathrm{CMA}_{0.6}-\mathrm{co}-\mathrm{Bz} \mathrm{MA}_{0.4}\right)_{150}$ & $11 \mathrm{mg}\left(5 \times 10^{-3} \mathrm{mmol}\right)$ & $130 \mathrm{mg}, 0.45 \mathrm{mmol}$ & $\mathrm{BzMA}(53 \mathrm{mg}, 0.30 \mathrm{mmol})$ & $1660 \mathrm{mg}$ & $3.01 \times 10^{-3} \mathrm{mmol} / \mathrm{g}$ & 98 & 97 \\
\hline $\mathrm{PEO}_{45}-b-\mathrm{P}\left(\mathrm{CMA}_{0.6}-\mathrm{co}-\mathrm{Bz} \mathrm{MA}_{0.4}\right)_{200}$ & $11 \mathrm{mg}\left(5 \times 10^{-3} \mathrm{mmol}\right)$ & $173 \mathrm{mg}, 0.60 \mathrm{mmol}$ & BzMA (70mg, 0.40mmol) & $1660 \mathrm{mg}$ & $3.01 \times 10^{-3} \mathrm{mmol} / \mathrm{g}$ & 96 & 98 \\
\hline $\mathrm{PEO}_{45}-b-\mathrm{P}\left(\mathrm{CMA} \mathrm{A}_{0.6} \text {-Co-ACMAE } \mathrm{E}_{0.4}\right)_{80}$ & $11 \mathrm{mg}\left(5 \times 10^{-3} \mathrm{mmol}\right)$ & $69 \mathrm{mg}, 0.24 \mathrm{mmol}$ & ACMAE (53 mg, $0.16 \mathrm{mmol})$ & $1660 \mathrm{mg}$ & $3.01 \times 10^{-3} \mathrm{mmol} / \mathrm{g}$ & 100 & 100 \\
\hline $\mathrm{PEO}_{45}-b-\mathrm{P}\left(\mathrm{CMA}_{0.6} \text {-co-ACMAE } E_{0.4}\right)_{100}$ & 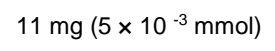 & $86 \mathrm{mg}, 0.30 \mathrm{mmol}$ & ACMAE (67 mg, $0.20 \mathrm{mmol})$ & $1660 \mathrm{mg}$ & $3.01 \times 10^{-3} \mathrm{mmol} / \mathrm{g}$ & 100 & 100 \\
\hline $\mathrm{PEO}_{45}-b-\mathrm{P}\left(\mathrm{CMA}_{0.6}-\mathrm{co}-\mathrm{ACMAE}_{0.4}\right)_{120}$ & $11 \mathrm{mg}\left(5 \times 10^{-3} \mathrm{mmol}\right)$ & $104 \mathrm{mg}, 0.36 \mathrm{mmol}$ & ACMAE (80 mg, $0.24 \mathrm{mmol})$ & $1660 \mathrm{mg}$ & $3.01 \times 10^{-3} \mathrm{mmol} / \mathrm{g}$ & 99 & 100 \\
\hline $\mathrm{PEO}_{45}-b-\mathrm{P}\left(\mathrm{CMA}_{0.6}-\mathrm{co}-\mathrm{ACMAE}_{0.4}\right)_{150}$ & $11 \mathrm{mg}\left(5 \times 10^{-3} \mathrm{mmol}\right)$ & $130 \mathrm{mg}, 0.45 \mathrm{mmol}$ & ACMAE (100mg, $0.30 \mathrm{mmol})$ & $1660 \mathrm{mg}$ & $3.01 \times 10^{-3} \mathrm{mmol} / \mathrm{g}$ & 99 & 100 \\
\hline $\mathrm{PEO}_{45}-b-\mathrm{P}\left(\mathrm{CM} \mathrm{A}_{0.6} \text {-co-ACMAE }_{0.4}\right)_{200}$ & $11 \mathrm{mg}\left(5 \times 10^{-3} \mathrm{mmol}\right)$ & $173 \mathrm{mg}, 0.60 \mathrm{mmol}$ & ACMAE (134mg, 0.40mmol) & $1660 \mathrm{mg}$ & $3.01 \times 10^{-3} \mathrm{mmol} / \mathrm{g}$ & 99 & 100 \\
\hline
\end{tabular}

The concentration of the polymer chain is roughly considered to be the same as the concentration of the PEO $45-\mathrm{CPADB}^{\mathrm{b}}{ }^{\mathrm{b}} \mathrm{The}$ conversion of CMA and comonomer (DIPEMA, BzMA or ACMAE) obtained via ${ }^{1} \mathrm{H}$ NMR spectra. 

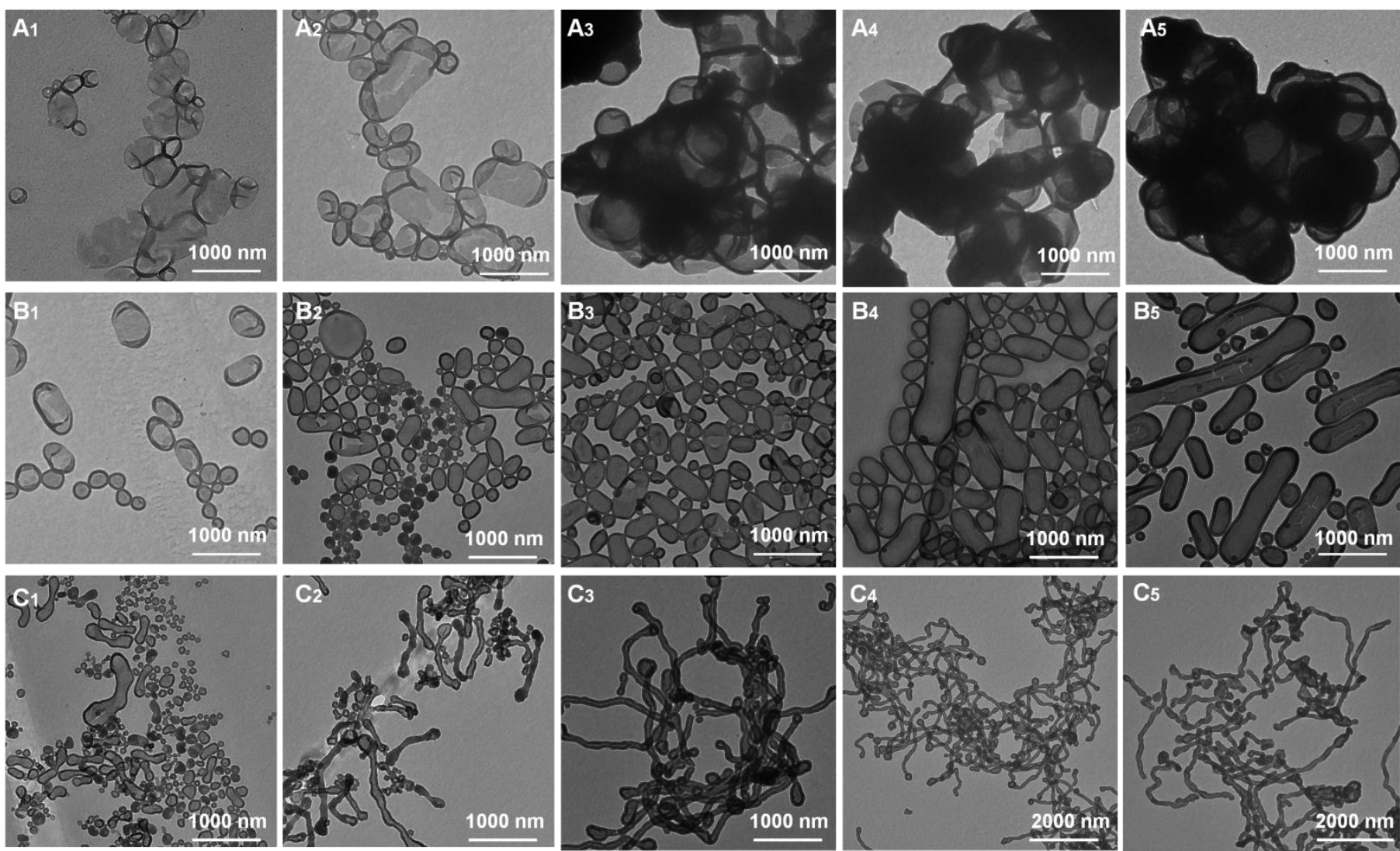

Fig. S36 RAFT dispersion copolymerization of CMA and DIPEMA/BzMA/ACMAE to adjust the aromatic interaction of solvophobic blocks (the molar content of the CMA units in the solvophobic blocks is fixed at 60\%). TEM images of $\left(A_{1}\right)$ PEO ${ }_{45}-b$-P $\left(C M A_{0.6}-C o-D I P E M A_{0.4}\right)_{80}, \quad\left(A_{2}\right)$ PEO ${ }_{45}-b-P\left(C M A_{0.6}-C o-D I P E M A_{0.4}\right)_{100}, \quad\left(A_{3}\right)$

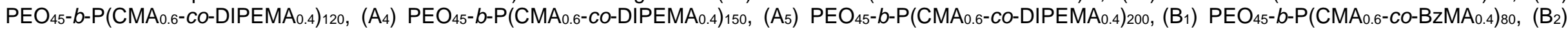

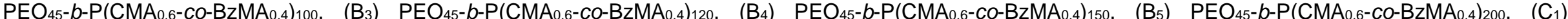

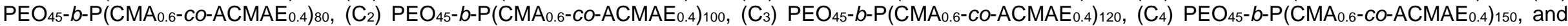
(C) $\mathrm{PEO}_{45}-b-\mathrm{P}\left(\mathrm{CMA}_{0.6}-\mathrm{CO}-\mathrm{ACMAE_{0.4 }}\right)_{200}$. The subscript number is the DP of the corresponding block. 
A

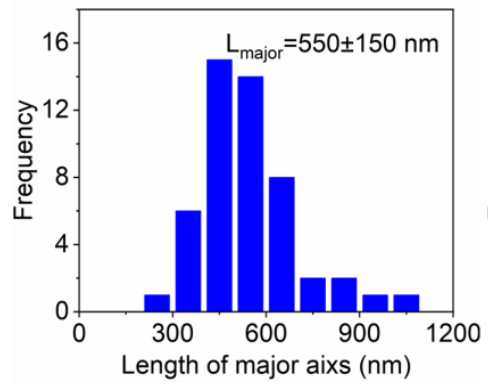

B

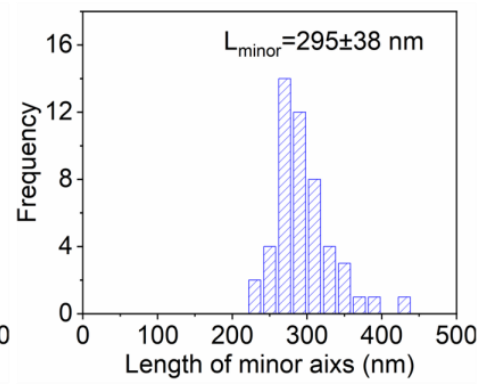

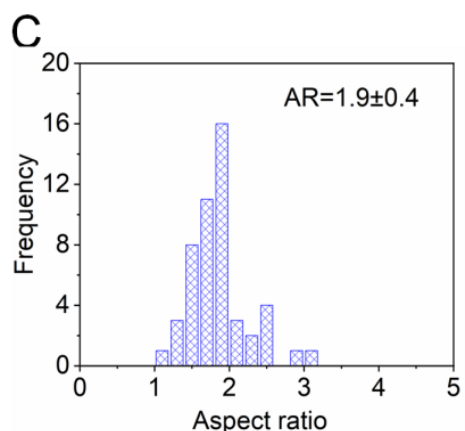

Fig. S37 Representative histograms for the (A) major axis lengths, (B) minor axis lengths (width) and $(\mathrm{C})$ aspect ratios of the PEO45-b-P(CMA0.6-co-BzMA0.4) 120 nanotubes obtained at the chain concentration of $3.01 \times 10^{-3} \mathrm{mmol} / \mathrm{g}$. Statistical analyses $(n=50)$ of the populations of these nanotubes on the basis of TEM observations using an open source image processing software (ImageJ).
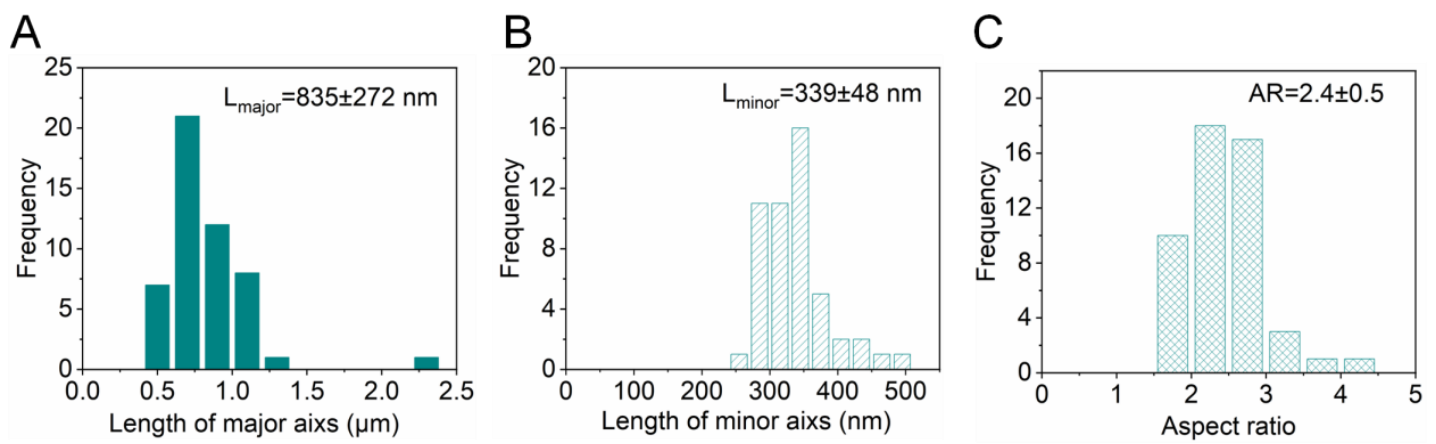

Fig. S38 Representative histograms for the (A) major axis lengths, (B) minor axis lengths (width) and $(\mathrm{C})$ aspect ratios of the

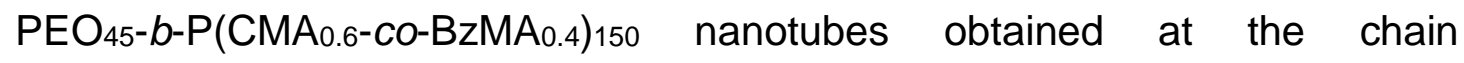
concentration of $3.01 \times 10^{-3} \mathrm{mmol} / \mathrm{g}$. Statistical analyses $(n=50)$ of the populations of these nanotubes on the basis of TEM observations using an open source image processing software (ImageJ). 

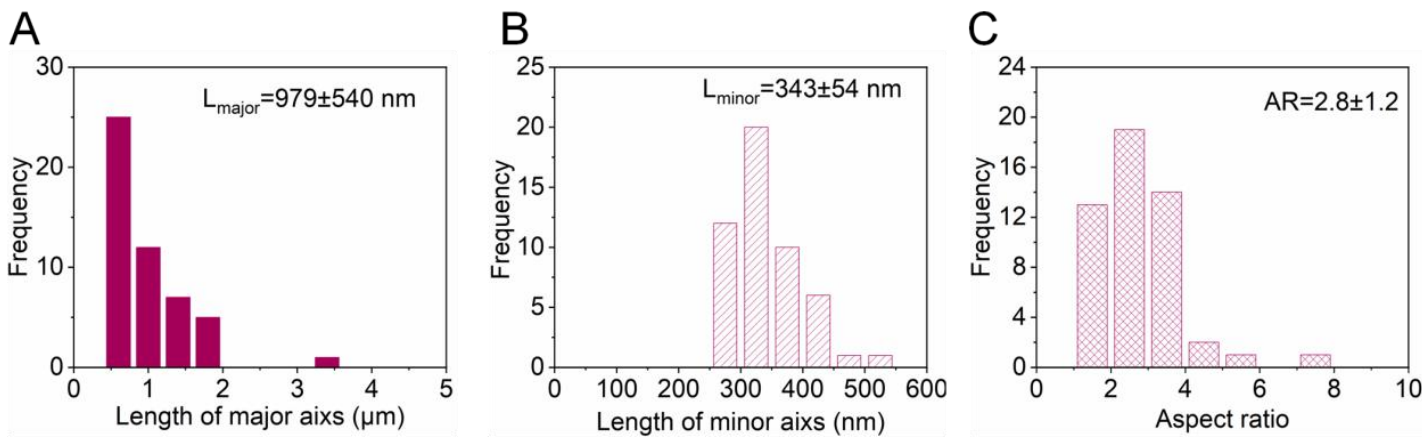

Fig. S39 Representative histograms for the (A) major axis lengths, (B) minor axis lengths (width) and (C) aspect ratios of the PEO $45-b-P(C M A 0.6-c o-B z M A 0.4)_{200}$ nanotubes obtained at the chain concentration of $3.01 \times 10^{-3} \mathrm{mmol} / \mathrm{g}$. Statistical analyses $(\mathrm{n}=50)$ of the populations of these nanotubes on the basis of TEM observations using an open source image processing software (ImageJ).
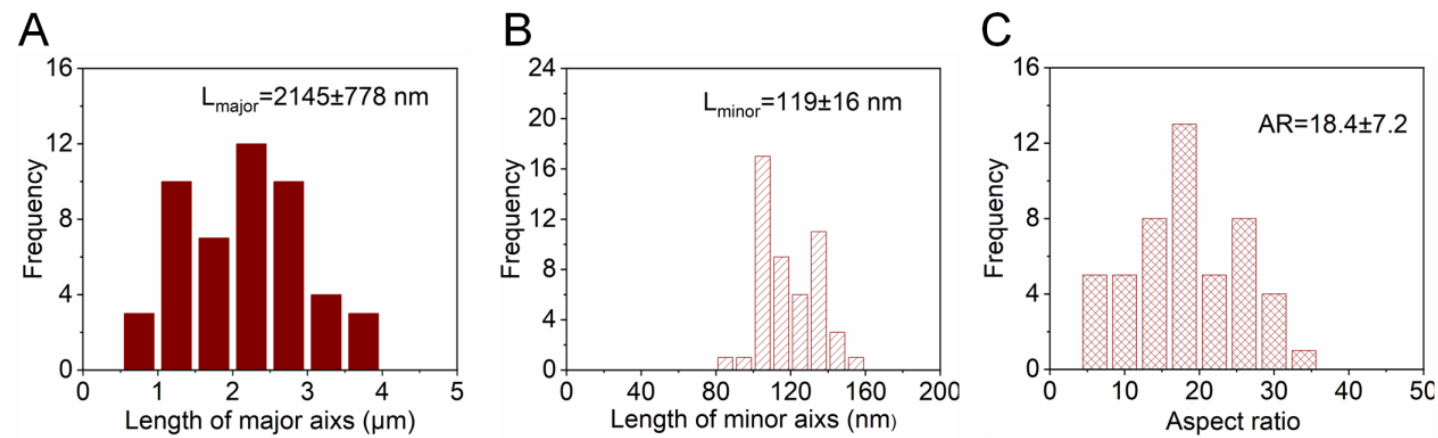

Fig. S40 Representative histograms for the (A) major axis lengths, (B) minor axis lengths (width) and (C) aspect ratios of the $\mathrm{PEO}_{45}-b-\mathrm{P}\left(\mathrm{CMA}_{0.6}-\mathrm{Co}-\mathrm{ACMAE}_{0.4}\right)_{120}$ nanotubes obtained at the chain concentration of $3.01 \times 10^{-3} \mathrm{mmol} / \mathrm{g}$. Statistical analyses $(n=50)$ of the populations of these nanotubes on the basis of TEM observations using an open source image processing software (ImageJ). 
A

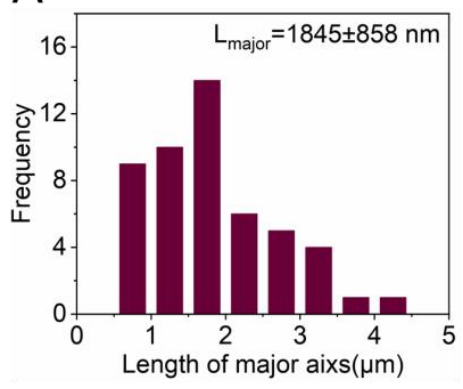

B

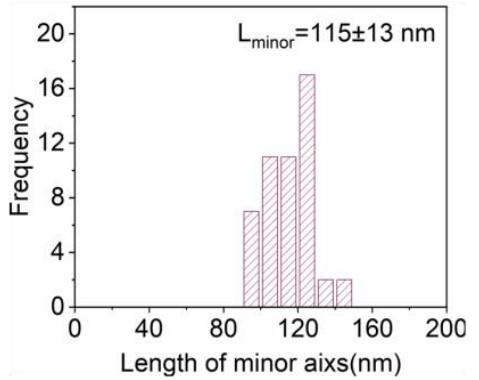

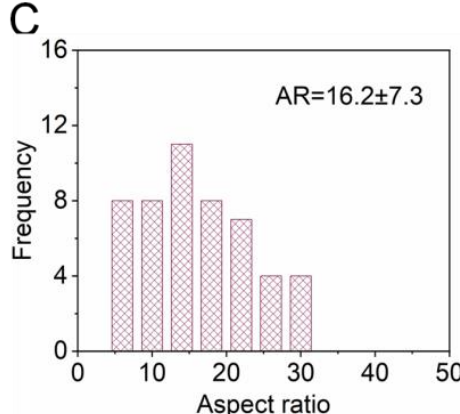

Fig. S41 Representative histograms for the (A) major axis lengths, (B) minor axis lengths (width) and (C) aspect ratios of the

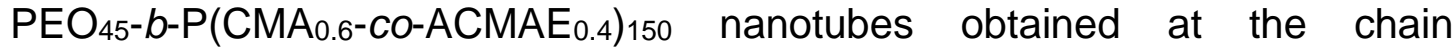
concentration of $3.01 \times 10^{-3} \mathrm{mmol} / \mathrm{g}$. Statistical analyses $(n=50)$ of the populations of these nanotubes on the basis of TEM observations using an open source image processing software (ImageJ).
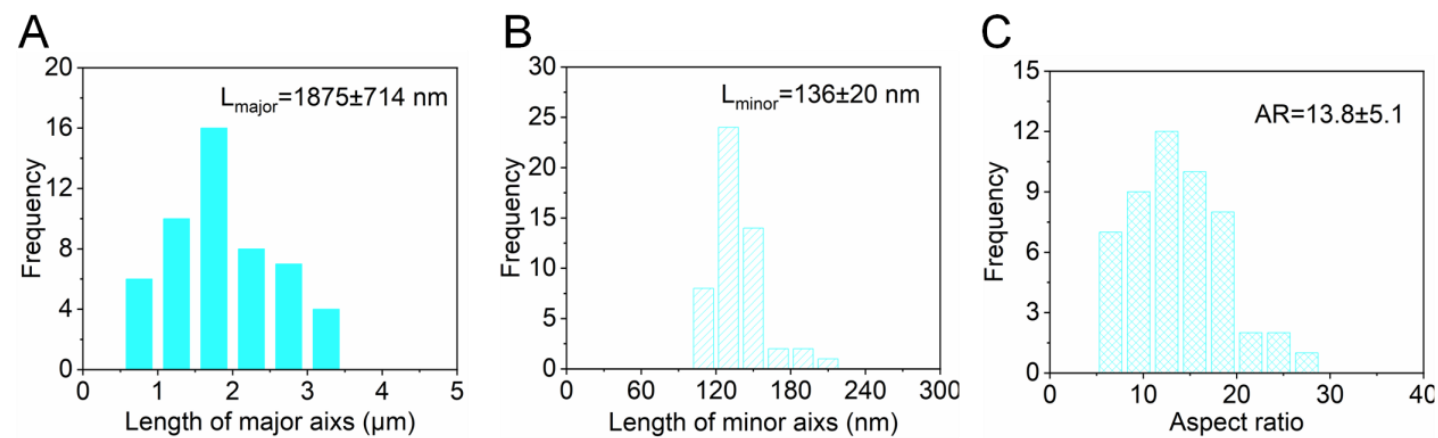

Fig. S42 Representative histograms for the (A) major axis lengths, (B) minor axis lengths (width) and (C) aspect ratios of the

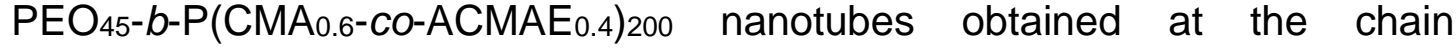
concentration of $3.01 \times 10^{-3} \mathrm{mmol} / \mathrm{g}$. Statistical analyses $(n=50)$ of the populations of these nanotubes on the basis of TEM observations using an open source image processing software (ImageJ). 